\title{
Convexidade e Sistemas Dinâmicos em Variedades Riemannianas
}

\author{
VALKIRIA ELIZABETH TEIXEIRA
}

Orientador:

PROF. DR. PAOLO PICCIONE

\author{
Dissertação apresentada ao \\ Instituto de Matemática e Estatística \\ da \\ Universidade de São Paulo \\ para \\ obtenção do grau \\ de \\ mestre em Matemática. \\ Área de concentração: Geometria Diferencial
}

- São Paulo, julho de 2002 -

Este trabalho contou com apoio financeiro da CAPES. 


\section{Título: \\ Convexidade e Sistemas Dinâmicos em Variedades Riemannianas}

São Paulo, 30 de julho de 2002.

Banca examinadora:

Prof. Dr. Paolo Piccione

IME-USP

Prof. Dr. Renato Hyuda de Luna Pedrosa

IMECC-UNICAMP

Profa. Dra. Rosa Maria dos Santos Barreiro Chaves

IME-USP 

Em memória

do meu pai Edgard e do meu irmão David Eduardo 


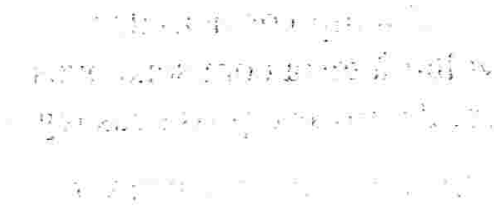




\section{Aulas de Vôo}

O conhecimento

caminha lento feito lagarta.

Primeiro não sabe que sabe

e voraz contenta-se com cotidiano orvalho

deixado nas folhas vívidas das manhãs.

Depois pensa que sabe

e se fecha em si mesmo: faz muralhas cava trincheiras, ergue barricadas.

Defendendo o que pensa saber levanta certezas em forma de muro orgulha-se de seu casulo.

Até que maduro explode em vôos

rindo do tempo em que imaginava saber ou guardava preso o que sabia.

Voa alto sua ousadia

reconhecendo o suor dos séculos

no orvalho de cada dia.

Mesmo o vôo mais belo

descobre um dia não ser eterno.

É tempo de acasalar

voltar à terra com seus ovos

à espera de novas e prosaicas lagartas.

O conhecimento é assim ri de si mesmo

e de suas certezas.

É meta de forma

metamorfose

movimento

fluir do tempo

que tanto cria como arrasa

a nos mostrar que para o vôo

é preciso tanto o casulo

como a asa.

Mauro Luis Iasi

in: Processo de Consciência - CPV (2001) 

"Como pode a Matemática, sendo acima de tudo um produto do pensamento humano, independente da experiência, se adaptar tão admiravelmente à realidade objetiva?"

"Em toda construção abstrata há um resíduo intuitivo (da experiência concreta) que é impossível eliminar."

Gonseth (1926)

"Não há ramo da Matemática, por abstrato que seja, que não possa um dia vir a ser aplicado aos fenômenos do mundo real."

Lobachevsky

in: Nilson José Machado, "Matemática e realidade: análise dos pressupostos filosóficos que fundamentam o ensino da Matemática” 4a. ed. Cortez, 1997. 



\section{Agradecimentos}

Esta dissertação é um marco no caminho de retomada dos meus estudos em Matemática e sou profundamente grata às pessoas que tornaram viáveis as condições para sua elaboração, durante os últimos três anos e meio.

Ao professor Paolo Piccione agradeço a sua orientação, que me permitiu entrar em contato com o processo de construção do conhecimento matemático. Foi decisivo poder contar não só com seu "fazer" matemático rigoroso, com sua lucidez e objetividade, que já admirava desde as aulas de Geometria Diferencial, mas também com seu reconhecimento ao meu esfôrço, nem sempre produtivo, fazendo com que sempre tivesse um novo desafio ao meu alcance, um novo ânimo.

À professora Maria Cristina Barufi a quem agradeço ter redescoberto o prazer de estudar e saber Matemática, pelo curso de Álgebra Linear no verão de 1999.

À professora Rosa Maria Chaves que me "apresentou" à Geometria Riemanniana, e em cujas aulas o primeiro capítulo desta dissertação se baseia. Sou grata pela confiança que sempre demonstrou no meu desempenho e por seu parecer favorável à obtenção da minha bolsa de estudos.

Ao professor Eduardo Colli em cujas aulas sobre Equações Diferenciais adquiri conhecimentos que muito me auxiliaram neste estudo.

Ao professor Plínio Amarante Quirino Simões agradeço as aulas sobre Funções Analíticas que me motivaram a estudar Cálculo e Análise, as respostas às minhas muitas perguntas, o muito que aprendi, e que me fez decidir a ingressar no curso de Pós-Graduação.

Ao professor Daniel V. Tausk por sua disponibilidade em esclarecer minhas dúvidas sempre de modo detalhado, claro.

Ao Feijão, Pinho e Francisca pela atenção que sempre tiveram no atendimento aos meus pedidos.

À minha família e aos meus amigos por toda compreensão e incentivo apesar das minhas frequentes e longas "ausências" para estudar. 



\section{Resumo}

Estuda-se as soluções de um sistema dinâmico conservativo e autônomo, que satisfazem a condições de fronteira a dois valores, sob um ponto de vista enumerativo. Interpretando-se uma variedade Riemanniana completa $(M, g)$ como o espaço das configurações de um sistema mecânico, estas soluções representam as trajetórias de massas que se movem sob a ação de uma força conservativa com potencial $-V$.

Quer-se estabelecer condições suficientes, métricas e topológicas sobre $(M, g)$, e para o potencial $V$, que garantam a existência e a finitude do número de tais soluções, ligando dois pontos $p, q \in M$ que sejam não-conjugados em $M$ em um sentido conveniente. Assim, a noção de conjugação induzida por um sistema dinâmico geral é estudada em detalhe.

O principal resultado sobre finitude assume uma condição que pode ser considerada tanto métrica como topológica em $(M, g)$ : assume-se que $(M, g)$ admite uma função estritamente convexa. Para tanto, estuda-se a noção de convexidade em $(M, g)$ isto é, as propriedades, exemplos e construções de funções convexas definidas, inicialmente na reta e, em variedades Riemannianas. 



\begin{abstract}
We study the solutions of a conservative and autonomous dynamical system on a Riemannian manifold $(M, g)$ satisfying the two-point boundary condition, from an enumerative point of view. Interpreting $M$ as the configuration space of a mechanical system, then these solutions represent the trajectories of masses under the influence of a conservative force with potencial $-V$.

Our aim is to establish sufficient conditions on $(M, g)$ and $V$ to guarantee the existence and the finiteness of the number of such solutions joining two points fixed on $M$ that are non conjugate in a suitable sense. Thus, the notion of conjugacy induced by a general dynamical system on $M$ is discussed in detail.

The main finiteness result is proved under the assumption that $(M, g)$ admits a non trivial convex function which is non increasing on the flow lines of the gradient of $V$; the notion of convexity on $(M, g)$ is studied i.e., the properties, examples and constructions of convex functions in Riemannian manifolds, in order to show that this assumption can be considered both topological and metrical conditions on $(M, g)$.
\end{abstract}





\section{Sumário}

Prefácio

Introdução $\quad$ xxi

Capítulo 1. Variedades Riemannianas 1

1. Métrica, Conexão e Curvatura Riemannianas 1

2. Gradiente e Hessiano de funções definidas em variedades Riemannianas 11

3. Segunda variação da Energia e conjugação ao longo de geodésicas 14

4. Completude 29

Capítulo 2. Convexidade em Variedades Riemannianas 35

1. Funções convexas em $\mathbb{R} \quad 35$

2. Funções convexas em variedades Riemannianas 44

Capítulo 3. Sistemas Dinâmicos Conservativos em Variedades Riemannianas 65

1. Caracterização do sistema dinâmico (3.1) com condições de fronteira

2. Conjugação ao longo das soluções de (3.1) e V-Campos de Jacobi 71

Capítulo 4. Finitude de Soluções de Sistemas Dinâmicos 79

1. Finitude de soluções do sistema dinâmico (3.1), satisfazendo (3.2) 79

2. Finitude de soluções do problema diferencial em que o potencial é nulo 86

3. Outros critérios de regularidade do sistema dinâmico (3.1) e Exemplos 89

Referências Bibliográficas $\quad 93$ 



\section{Prefácio}

Este estudo baseia-se nos artigos [5] e [6] que discutem as relações entre a noção de convexidade e a finitude do número de soluções ligando dois pontos fixos de um sistema dinâmico em uma variedade Riemanniana completa $(M, g)$. No primeiro prova-se que o número de geodésicas ligando dois pontos não-conjugados é finito sob a hipótese de que $(M, g)$ admite uma função convexa não-trivial e no segundo, generaliza-se este resultado para sistemas dinâmicos conservativos e autônomos.

O texto desta dissertação está organizado em quatro capítulos: 1 - Variedades Riemannianas, 2 - Convexidade, 3 - Sistemas Dinâmicos Conservativos e 4 - Finitude de soluções de Sistemas Dinâmicos.

No primeiro estabelece-se os conceitos e resultados fundamentais da Geometria Riemanniana que embasam a teoria desenvolvida nos capítulos seguintes: Métrica, conexão e curvatura Riemannianas; completude de $(M, g)$; operadores diferenciais Gradiente e Hessiano de funções definidas em $M$; geodésicas, vizinhanças normais, variações da Energia, conjugação e Campos de Jacobi, Índice de Morse.

No segundo capítulo apresenta-se as propriedades básicas e exemplos de funções convexas definidas em variedades Riemannianas completas.

No terceiro caracteriza-se um sistema dinâmico conservativo e autônomo (3.1), objeto deste estudo, e mostra-se que aquelas soluções de (3.1) que satisfazem a condições de fronteira a dois valores (3.2) são os pontos estacionários do funcional Lagrangiano, generalizando-se a teoria sobre o funcional Energia, desenvolvida na Seção 3 do Capítulo 1. Além disso, estabelece-se a noção de conjugação ao longo das soluções de (3.1), satisfazendo (3.2), e de V-Campos de Jacobi.

O capítulo 4 é destinado à discussão das condições suficientes sobre $(M, g) \mathrm{e}$ para o potencial $V$ que garantam, além da existência de soluções, a regularidade do sistema dinâmico (3.1) e portanto, a obtenção do resultado principal sobre a finitude do número de soluções de (3.1) satisfazendo (3.2). Na Seção 3 são discutidos outros dois critérios de regularidade para o sistema dinâmico (3.1), assim como exemplos de onde esta teoria se aplica, e destacando-se ainda o caso particular em que o potencial $V$ é nulo. 



\section{Introdução}

Considere a seguinte equação diferencial definida em uma variedade Riemanniana $(M, g)$ completa

$$
\text { (3.1) } \frac{D \dot{x}}{d t}=-\nabla V(x)
$$

onde $V: M \rightarrow \mathbb{R}$ é uma função de classe $C^{2}$.

Interpreta-se $M$ como o espaço das configurações de um sistema mecânico onde massas se movem sob a ação de uma força conservativa com potencial $-V$.

Fixados um ponto $p$ em $M$ e um valor positivo para o parâmetro tempo $T$, as soluções de (3.1) são curvas $x:[0, T] \rightarrow M$ em $M$ que representam as trajetórias do campo gradiente de $V$ partindo de $p$ no instante inicial e chegando na posição $x(T)=q$ após o tempo $T$. Assim, fixados $p$ e $q$ em $M$, interessa-nos analisar as soluções de (3.1) que satisfazem as chamadas condições de fronteira:

$$
(3.2) x(0)=p \text { e } x(T)=q .
$$

As equações (3.1) e (3.2) constituem o problema diferencial com condições de fronteira a dois valores estudado com o objetivo principal de estabelecer-se condições métricas e topológicas sobre $(M, g)$ e condições suficientes para o potencial $V$ que garantam a existência e a finitude do número de soluções de (3.1) que satisfazem as condições de fronteira (3.2), para quase todo par de pontos $p, q$ em $M$.

O sistema dinâmico (3.1) é conservativo ou seja, a energia total do sistema é constante ao longo de cada trajetória $x$, e autônomo isto é, o conjunto das soluções de (3.1) é invariante por reparametrizações que envolvam translações ou reversões do tempo.

Os pontos que são conjugados em relação a um sistema dinâmico tem um papel crucial na teoria. Em linhas gerais, dois pontos $p$ e $q$ são conjugados relativamente ao sistema (3.1) se existe uma homotopia $\left\{x_{s}\right\}_{s \in]-\epsilon, \epsilon[}$ de soluções de (3.1) satisfazendo as condições de fronteira $x_{s}(0)=p$ e $x_{s}(T)=q$, com campo variacional $J$ ao longo de uma solução $x$ e que é um V-Campo de Jacobi (Lema 3.11).

As soluções de um sistema conservativo são os pontos críticos, ditos estacionários, do funcional Lagrangiano $\mathcal{L}_{T}$ definido no espaço de todas as curvas $C^{1}$ ligando $p$ a $q$ em $\mathrm{M}$, denotado por $\Omega_{p, q}([0, T], M)$. Prova-se que toda solução $x$ de 
(3.1) satisfazendo (3.2) é um ponto crítico não-degenerado para o funcional $\mathcal{L}_{T}$ desde que $p$ e $q$ sejam pontos não-conjugados sobre $[0, T]$ relativamente a (3.1), conforme a Proposição 3.14. Ou seja, $\mathcal{L}_{T}$ é uma função de Morse em $\Omega_{p, q}([0, T], M)$ se $p$ e $q$ são não-conjugados,.

A principal condição técnica para aplicar-se a Teoria de Morse em uma variedade Hilbertiana é a condição de compacidade de Palais-Smale. Por esta razão assume-se o crescimento sub-quadrático da função potencial $V$. Daí, nesta situação, o funcional Lagrangiano $\mathcal{L}_{T}$ é limitado inferiormente e satisfaz as condições de compacidade de Palais-Smale em $\Omega_{p, q}([0, T], M)$ (Lema 4.4), garantindo-se assim, a existência de soluções de (3.1) ligando dois pontos $p$ e $q$ não-conjugados.

Fixando-se o ponto $p$ em $M$, considera-se uma partição de $M$ formada pelos conjuntos $\sigma_{i}$ dos pontos $q$ em $M$ para os quais existem exatamente $i$ soluções de (3.1), $i=0, \cdots,+\infty$. Toma-se este modelo em $M$, análogo à Decomposição Focal do plano introduzido por Peixoto [17], para estabelecer equivalentemente condições tais que $\sigma_{\infty}$ tenha medida nula em $M$ isto é, tal que para quase toda escolha de $q$, o número de trajetórias do sistema dinâmico (3.1) que começam em $p$ e terminam em $q$, após um tempo $T$ fixado, seja finito. Tal resultado é obtido a partir do fato de que o conjunto dos pontos $q \in M$ que são conjugados a $p$ sobre $[0, T]$ relativamente a (3.1) tem medida nula em $M$. A prova desta afirmação (Proposição 3.15) utiliza uma técnica análoga ao caso da conjugação através de geodésicas onde os pontos conjugados a um dado $p$ em $M$ são caracterizados como os valores críticos da aplicação exponencial em $p$ e a conclusão é obtida com o Teorema de Sard.

O principal resultado sobre finitude usa uma hipótese que pode ser considerada tanto topológica como métrica em $(M, g)$ quer dizer, assume-se que $(M, g)$ admite uma função estritamente convexa. A existência de funções convexas em $(M, g)$ não depende exclusivamente da métrica $g$ em $M$, mas também da topologia de $M$. Em particular, variedades Riemannianas compactas não admitem funções convexas nãoconstantes. Assim, somente a existência de uma função convexa não-constante não tem implicações na estrutura topológica da variedade exceto quanto às propriedades de não-compacidade.

Sabe-se, por um resultado de Green e Shiohama [8], que em toda variedade nãocompacta $M$ existe uma métrica Riemanniana completa $g$ em $M$ e uma função nãoconstante $C^{\infty}, F: M \rightarrow \mathbb{R}$ que é convexa em $(M, g)$. Assim, funções convexas não-triviais podem ser obtidas em toda variedade não-compacta, em relação a uma determinada métrica Riemanniana, e se esta função admitir um ponto crítico nãodegenerado, terá implicações importantes na estrutura topolọgica da variedade. É 
mostrado que se $(M, g)$ admite uma função convexa que tem um ponto de mínimo não-degenerado então $M$ é contrátil e difeomorfa a $\mathbb{R}^{m}$.

Funções convexas aparecem naturalmente em variedades com curvatura nãopositiva, por exemplo, se uma variedade Riemanniana simplesmente conexa, completa e não-compacta, admite um polo $p_{0}$ tal que sua curvatura radial é não-positiva em todo ponto $p$ de $M$ então a função $d(p)=\operatorname{dist}^{2}\left(p, p_{0}\right)$ é estritamente convexa em $M$ (Exemplo 2.48). Mas também toda variedade não-compacta com curvatura seccional não-negativa admite uma função de Busemann que é convexa (Exemplo 2.43). Portanto não há relação entre a curvatura de $(M, g)$ e a existência de funções convexas definidas em $M$.

Considera-se inicialmente uma redução do problema, mostrando-se um resultado particular, "local", sobre a finitude do número de soluções do sistema dinâmico (3.1) ligando dois pontos $p$ e q não-conjugados em uma variedade Riemanniana completa $(M, g)$ que admite uma função estritamente convexa: para todo subconjunto compacto $K \subset M$ com $p, q \in K$, há apenas um número finito de soluções com imagem em $K$ (Proposição 4.5). Para prová-lo, começa-se com um lema sobre a convergência uniforme, $C^{2}$, de sequências de curvas cuja aceleração covariante vai a zero uniformemente. Esse resultado permite assumir que, se as soluções de um sistema dinâmico conservativo estiverem contidas em um subconjunto compacto de $M$ e com energia divergindo, então estas soluções tendem a geodésicas. Quer dizer, as trajetórias de partículas com energia cinética muito grande tendem a ser "linhas retas", sem fazer caso de forças gravitacionais. Nesta situação, pode-se utilizar as técnicas-padrão de convexidade em $M$.

Analisa-se a seguir sob que condições tem-se finitude no número de todas as soluçôes do sistema dinâmico (3.1) satisfazendo as condições de fronteira (3.2). O resultado central nesta direção está baseado num controle adequado do crescimento ao infinito da função convexa $F$ ao longo das linhas de fluxo do campo gradiente do potencial $V$. Na verdade, só é possível passar do resultado "local" para o "global", quando os sistemas dinâmicos são regulares isto é, tem a propriedade de que: todas as suas soluções com extremos dentro de um conjunto compacto não saem de um subconjunto compacto de $M$, possivelmente maior. A existência de uma função estritamente convexa em $M$ não-crescente ao longo das curvas integrais de $\nabla V$ é uma condição suficiente para garantir a regularidade do sistema dinâmico (3.1) e pode-se, então, mostrar na Proposição 4.7, nosso resultado principal: Dadas uma variedade Riemanniana completa $(M, g)$ e $V: M \rightarrow \mathbb{R}$ uma aplicação $C^{2}, e$ assumindo-se que $M$ admita uma função fortemente convexa $F: M \rightarrow \mathbb{R}$ tal que $F$ tem um ponto de mínimo em $M$ e F é não-crescente em toda linha de fluxo do $\nabla V$; 
se $p$ e q são não-conjugados sobre $[0, T]$ relativamente a (3.1) então existe apenas um número finito de soluções do problema (3.1) e (3.2).

Sob estas mesmas hipóteses prova-se que o conjunto $\sigma_{\infty}$ tem medida nula em $M$ e além disso, Giannoni [6, Seção 5] prova também que, assumindo-se o crescimento sub-quadrático de $V$, o número de soluções ligando dois pontos fixados é genericamente ímpar ou seja, os conjuntos $\sigma_{2 i}$ tem medida nula para todo $i>0$.

Um problema mais simples, também interessante para métricas não planas, pode ser útil para entender-se nosso resultado principal já que foi a motivação inicial para utilizar-se a hipótese de convexidade. É o caso em que o potencial $V$ é nulo isto é, quando o sistema (3.1) se reduz à equação de geodésicas em $(M, g)$ ligando $p$ a $q$. Provavelmente, o primeiro resultado sobre finitude para esta situação é o Teorema de Hadamard-Cartan (1.58), que estabelece que se $M$ é simplesmente conexa, $(M, g)$ é completa, com curvatura seccional não-positiva, então entre quaisquer dois pontos existe uma línica geodésica.

A Proposição 4.7 dá-nos um resultado finito para geodésicas ligando dois pontos fixos não-conjugados em $M$, que admite uma função estritamente convexa com um ponto de mínimo. Este resultado, provado diretamente na Proposição 4.12, pode ser usado em Relatividade Geral para estudar o chamado efeito G.L. das lentes gravitacionais; em termos geométricos, este fenômeno corresponde à existência de múltiplas geodésicas lightlike ligando um evento $p$ e um observador $\gamma$ fixados em uma variedade Lorentziana [5, Seção 4]. É possível obter-se generalizações deste resultado para métricas Lorentzianas não-estáticas, desenvolvendo-se uma teoria análoga à do Capítulo 3 para funcionais Lagrangianos de forma geral $\mathcal{L}_{T}(x)=\int_{a}^{b} \mathcal{L}(t, x, \dot{x}) d t$. O caso em que a métrica Lorentziana é estacionária isto é, independente do tempo, é estudado em Giannoni [7]. 


\section{CAPíTULO 1}

\section{Variedades Riemannianas}

\section{Métrica, Conexão e Curvatura Riemannianas}

Diz-se que $M$ é uma variedade diferenciável, $C^{\infty}$, de dimensão $m$ se $M$ é um espaço topológico que:

(1) satisfaz ao Axioma da separação de Hausdorff: para todo par $x, y \in M$, existem abertos $U$ e $V, x \in U, y \in V$ tal que $U \cap V=\emptyset$;

(2) satisfaz o segundo Axioma da Enumerabilidade, isto é, $M$ admite uma base enumerável de abertos;

(3) é localmente euclidiano de dimensão $m$ : cada $p \in M$ admite uma vizinhança aberta homeomorfa a um aberto do $\mathbb{R}^{m}$;

(4) possui uma estrutura diferenciável $\mathcal{F}$ de classe $C^{\infty}$ ou seja, uma coleção de sistemas de coordenadas $\left(U_{\alpha}, \varphi_{\alpha}\right)_{\alpha \in A}$, chamado de $o$ atlas de $M$, tal que

(a) $\bigcup_{\alpha \in A} U_{\alpha}=M$;

(b) $\varphi_{\alpha} \circ \varphi_{\beta}^{-1}: \varphi_{\beta}\left(U_{\alpha} \cap U_{\beta}\right) \rightarrow \varphi_{\alpha}\left(U_{\alpha} \cap U_{\beta}\right)$ é uma aplicação $C^{\infty}$ entre abertos do $\mathbb{R}^{m}$;

(c) $\mathcal{F}$ é maximal com respeito a (b), isto é, se $(U, \varphi)$ é um sistema de coordenadas de $M$, e $\varphi \circ \varphi_{\alpha}^{-1}$ e $\varphi_{\alpha} \circ \varphi^{-1}$ são de classe $C^{\infty}$, para todo $\alpha \in A$, então $(U, \varphi) \in \mathcal{F}$.

O espaço tangente de uma variedade diferenciável $M$ no ponto $p$ será denotado por $T_{p} M . \operatorname{Se}(U, \varphi)$ é um sistema de coordenadas em $p, \varphi(p)=x=\left(x_{1}, x_{2}, \cdots, x_{m}\right)$, $\left\{\left(\frac{\partial}{\partial x_{i}}\right)_{0}\right\}_{i=1, \cdots, m}$ é a base natural de $T_{p} M$ associada a $(U, \varphi)$ e $\operatorname{dim} T_{p} M=m$.

Denota-se por $\mathcal{D}(p)=C^{\infty}(p)$ o conjunto das funções $f: U \rightarrow \mathbb{R}$ diferenciáveis em $p \in U \subset M$; por $\mathcal{D}(M)$ o conjunto das funções diferenciáveis em $M$.

Se $f \in \mathcal{D}(p)$ então

$$
\left(\frac{\partial}{\partial x_{i}}\right)[f]=\left.\frac{\partial}{\partial x_{i}}\left(f \circ \varphi^{-1}\right)\right|_{x=\varphi(p)} .
$$

Se $\alpha:]-\epsilon, \epsilon[\rightarrow M$ é uma curva diferenciável em $M \operatorname{com} \alpha(0)=p$ e $\left.\frac{d}{d t} \alpha(t)\right|_{t=0}=\dot{\alpha}(0)=v \in T_{p} M$, então

$$
v[f]=\left.\frac{d}{d t}(f \circ \alpha)(t)\right|_{t=0}=\mathrm{d} f_{p}(v)
$$

onde $\mathrm{d} f_{p}(v)$ é dita a diferencial de $f$ em $p$ na direção de $v$.

([2, Capítulo IV]) 
$T_{p} M$ é uma fibra do fibrado tangente $T M=\left\{(p, v): p \in M\right.$ e $\left.v \in T_{p} M\right\}$ munido de uma estrutura diferenciável (de dimensão $2 \mathrm{~m}$ ). Quer dizer, existe uma projeção natural $\pi: T M \rightarrow M$ tal que $\pi^{-1}(p)=\{p\} \times T_{p} M$ é a fibra no ponto $p$.

DEFINIÇão 1.1. Um campo de vetores $X$, definido em um aberto $U \subset M$ é uma aplicação $X: U \subset M \rightarrow T M$ tal que $\pi \circ X=\left.I d\right|_{U}$.

Diz-se que $\mathfrak{X}(M)$, o conjunto dos campos diferenciáveis em $M$, munido de uma estrutura de espaço vetorial sobre $\mathbb{R}$, é uma álgebra de Lie se admite também o produto $[X, Y]$ satisfazendo as seguintes propriedades, para todo $X, Y, Z \in \mathfrak{X}(M)$ :

(1) é bilinear sobre $\mathbb{R}$;

(2) $[X, Y]=-[Y, X]$;

(3) $[X,[Y, Z]]+[Y,[Z, X]]+[Z,[X, Y]]=0$, a chamada Identidade de Jacobi.

Prova-se que $[X, Y]$, conhecido como o Colchete de Lie de $X$ e $Y$, define um campo em $M$ que atua do seguinte modo:

$$
\begin{aligned}
{[X, Y](p): \mathcal{D}(p) } & \rightarrow \mathbb{R} \\
f & \rightarrow[X, Y](p)[f]=X_{p}[Y f]-Y_{p}[X f]
\end{aligned}
$$

onde $X_{p}=X(p) \in T_{p} M ; X[f](p)=X_{p}[f]$ e $X f=X[f]: M \rightarrow \mathbb{R}$.

DEFINIÇÃO 1.2. Uma métrica Riemanniana é uma lei que faz corresponder a cada $p \in M$ uma aplicação $g_{p}: T_{p} M \times T_{p} M \rightarrow \mathbb{R}$ satisfazendo, para todo $u, v, w \in T_{p} M$ e $a \in \mathbb{R}$.

(1) $g(u, v)=g(v, u)$

(2) $g(u+v, w)=g(u, w)+g(v, w)$ e $g(a u, v)=a g(u, v)$

(3) g é definida positiva ou seja, é um produto interno em $T_{p} M: g(u, u) \geq 0 e$

$g(u, u)=0 \Longleftrightarrow u=0$. Esta propriedade garante que gé uma métrica nãodegenerada isto é, se $g(u, v)=0$ para todo $v \in T_{p} M$ então $u=0$.

(4) Se $X$ e $Y$ são campos diferenciáveis em um aberto $U \subset M$, então a função

$U \subset M \rightarrow \mathbb{R}$

$p \quad \rightarrow g_{p}(X(p), Y(p))$ é diferenciável em $U$.

Toda variedade diferenciável (paracompacta) possui uma métrica Riemanniana.

([2, pág. 193] e [3, Capítulo 1 - Proposição 2.10]).

Uma variedade diferenciável $M$ com uma métrica Riemanniana é dita uma variedade Riemanniana e denotada por $(M, g)$.

Diz-se que a métrica $g$ define uma estrutura pseudo-Riemanniana em $M$, ou que $(M, g)$ é uma variedade pseudo-Riemanniana, se valem (1), (2) e (4) da Definição 1.2; $g$ é não-degenerada isto é, para todo $p \in M$ e todo $v \in T_{p} M, v \neq 0$ existe $w \in T_{p} M$ tal que $g(v, w) \neq 0$; mas $g$ não é necessariamente definida positiva. 
OBSERVAÇÃo 1.3. Se $V$ é um espaço vetorial sobre $\mathbb{R}$, uma forma bilinear simétrica em $V$ é uma função $\mathbb{R}$-bilinear $b: V \times V \rightarrow \mathbb{R}$ satisfazendo

$b(v, w)=b(w, v)$ para todo $v, w \in V$.

É dita definida positiva (negativa) se dado $v \neq 0, b(v, v)>0(<0)$;

semidefinida positiva (negativa) quando $b(v, v) \geq 0(\leq 0)$ para todo $v \in V$.

Considere $V$ de dimensão finita $m$ e $\left\{e_{1}, \cdots, e_{m}\right\}$ uma base de $V$. A matriz simétrica $b_{i j}=b\left(e_{i}, e_{j}\right)$ é diagonalizável e, pelo Teorema de Sylvester, existe uma base de $V$ tal que a diagonal de $b_{i j}$ é dada apenas pelos autovalores $1,-1$ e 0 .

$\mathrm{O}$ índice $\nu$ de uma forma bilinear $b \mathrm{em} V$ é igual ao número de autovalores -1 . O número de l's é o co-índice de $b$ em $V$. Assim, $0 \leq \nu \leq m$. E $\nu=0$ se e somente se $b$ é semidefinida positiva.

Se $(U, \varphi=x)$ é um sistema de coordenadas na vizinhança de um ponto $p$ e $\left\{\left(\frac{\partial}{\partial x_{i}}\right)_{0}\right\}_{i=1, \cdots, m}$ a base associada de $T_{p} M$, as funções

$$
g_{i j}=g\left(\frac{\partial}{\partial x_{i}}, \frac{\partial}{\partial x_{j}}\right)
$$

são os coeficientes ou expressão da métrica no sistema de coordenadas dado.

Segue que: $g_{i j}=g_{j i}$ pois $g$ é uma forma bilinear simétrica em $T_{p} M$ por (1) e (2); $\operatorname{det}\left(g_{i j}\right) \neq 0$ pois $g$ é não-degenerada e logo a matriz $\left(g_{i j}\right)$ é invertível e as $g_{i j}$ são diferenciáveis em $U$ por (4). Como a matriz $\left(g_{i j}\right)$ é simétrica, existe uma base de $T_{p} M$ que a diagonaliza e tal que nenhum autovalor é nulo.

$\mathrm{O}$ valor $\nu$ do índice de uma métrica pseudo-Riemanniana $g_{p}$ em $T_{p} M$ comum a todo $p \in M$ é chamado de o índice de $M$. Se $\nu=0, M$ é uma variedade Riemanniana. Se $\nu=1, M$ é uma variedade Lorentziana. Mais precisamente:

DEFINIÇÃo 1.4. Uma métrica Lorentziana $g$ em uma variedade $M$ é uma forma bilinear simétrica não-degenerada $g_{p}: T_{p} M \times T_{p} M \rightarrow \mathbb{R}$ com assinatura, isto é, diagonal na forma $(-,+, \ldots .,+)$ em cada espaço tangente. Existem três classes de vetores tangentes:

$v \in T_{p} M$ é do tipo nulo ("null" ou "lightlike") se $g(v, v)=0$ e $v \neq 0$,

espacial ("spacelike") se $g(v, v)>0$ ou $v=0$,

temporal ("timelike") se $g(v, v)<0$.

Um vetor não-espacial $(g(v, v) \leq 0$ e $v \neq 0)$ é também chamado de causal.

Daqui em diante, assume-se $(M, g)$ uma variedade conexa, com estrutura diferenciável $C^{\infty}$ e Riemanniana, embora os conceitos e propriedades considerados sejam válidos para variedades pseudo-Riemannianas em geral. 
Espaços topológicos são equivalentes quando homeomorfos, mas para se falar de equivalência entre variedades Riemannianas não basta que sejam difeomorfas, é necessário considerar as métricas e o conceito de isometria, que preserva a métrica no seguinte sentido:

DEFINIÇÃo 1.5. Sejam $\left(M, g_{1}\right)$ e $\left(N, g_{2}\right)$ variedades Riemannianas.

Um difeomorfismo $f: M \rightarrow N$ é uma

(I) isometria se $g_{2}\left(\mathrm{~d} f_{p}(u), \mathrm{d} f_{p}(v)\right)_{f(p)}=g_{1}(u, v)_{p}$ para todo $u, v \in T_{p} M e$ para todo $p \in M$;

(2) aplicação conforme se existe $\lambda: M \rightarrow \mathbb{R}^{+}$diferenciável tal que $g_{2}\left(\mathrm{~d} f_{p}(u), \mathrm{d} f_{p}(v)\right)_{f(p)}=\lambda(p) g_{1}(u, v)_{p}$ para todo $u, v \in T_{p} M$ e para todo $p \in M$.

DEFINIÇÃo 1.6. Seja $(M, g)$ uma variedade Riemanniana. Para todo $p \in M$ e $v \in T_{p} M$, o comprimento $\|v\|$ é definido por $\|v\|=g(v, v)^{\frac{1}{2}}$. Assim, ||.|| indica a norma induzida por $g$ em cada espaço tangente.

Se $\alpha:[a, b] \subset \mathbb{R} \rightarrow M$ é uma curva diferenciável e regular em $M$ isto é, $\dot{\alpha}(t) \neq 0$ para todo $t \in[a, b]$, o comprimento $l(\alpha)$ é dado por $l(\alpha)=\int_{a}^{b}\|\dot{\alpha}(t)\| d t$. $l(\alpha)$ é também denotado por $\mathcal{L}_{a}^{b}(\alpha)$, e independe da parametrização da curva $\alpha$.

$A$ função comprimento de arco $s$ da curva $\alpha$ é definida por

$$
\begin{aligned}
s:[a, b] & \rightarrow \mathbb{R} \\
t & \rightarrow s(t)=\int_{a}^{t}\|\dot{\alpha}(r)\| d r=\mathcal{L}_{a}^{t}(\alpha) .
\end{aligned}
$$

Se o parâmetro de uma curva é o próprio comprimento de arco, seu campo tangente é unitário, e é, então, chamada de curva normalizada.

Seja $(U, \varphi=x)$ um sistema de coordenadas $\operatorname{com} \alpha([a, b]) \subset U$.

$$
\begin{aligned}
\mathrm{Se} x_{i}(t) & =\left(x_{i} \circ \alpha\right)(t), \\
(\varphi \circ \alpha)(t) & =\left(x_{1}(t), \cdots, x_{m}(t)\right), \\
\alpha(t) & =\varphi^{-1}\left(x_{1}(t), \cdots, x_{m}(t)\right), \\
\dot{\alpha}(t) & =\sum_{i=1}^{m}\left(\frac{\partial \varphi^{-1}}{\partial x_{i}}\right)_{x(t)} \frac{d x_{i}}{d t}=\sum_{i=1}^{m} \frac{d x_{i}}{d t}\left(\frac{\partial}{\partial x_{i}}\right)_{\alpha(t)} .
\end{aligned}
$$

Segue da Definição 1.6 que

$$
\begin{aligned}
& \left(\frac{d s}{d t}\right)^{2}=g(\dot{\alpha}(t), \dot{\alpha}(t)) . \text { Então } \\
& \left(\frac{d s}{d t}\right)^{2}=g\left(\sum_{i=1}^{m} \frac{d x_{i}}{d t}\left(\frac{\partial}{\partial x_{i}}\right), \sum_{j=1}^{m} \frac{d x_{j}}{d t}\left(\frac{\partial}{\partial x_{j}}\right)\right)=\sum_{i, j=1}^{m} \frac{d x_{i}}{d t} \frac{d x_{j}}{d t} g\left(\frac{\partial}{\partial x_{i}}, \frac{\partial}{\partial x_{j}}\right) .
\end{aligned}
$$

Portanto gé dada como combinação linear das $g_{i j}$ e a expressão local da métrica $g$ é dada pelo quadrado do elemento de arco: $\mathrm{d} s^{2}=\sum_{i, j=1}^{m} g_{i j} \mathrm{~d} x_{i} \mathrm{~d} x_{j}$. 
DEFINIÇÃO 1.7. Uma conexão afim em uma variedade diferenciável $M$ é uma aplicação $\nabla: \mathfrak{X}(M) \times \mathfrak{X}(M) \rightarrow \mathfrak{X}(M)$ que satisfaz:

(1) $\nabla_{X+Y} Z=\nabla_{X} Z+\nabla_{Y} Z$

(2) $\nabla_{X}(Y+Z)=\nabla_{X} Y+\nabla_{X} Z$

(3) $\nabla_{f X} Y=f \nabla_{X} Y$

(4) $\nabla_{X} f Y=X[f] Y+f \nabla_{X} Y$

para todo $X, Y, Z \in \mathfrak{X}(M), f \in \mathcal{D}(M)$.

Dado $(U, x)$ um sistema de coordenadas local de $M$ em $p$, e sendo $\left\{X_{i}=\frac{\partial}{\partial x_{i}}\right\}_{i=1, \cdots, m}$ a base associada de $T_{p} M$, existem únicas funções $C^{\infty} \Gamma_{i j}^{k}: U \subset M \rightarrow \mathbb{R}$, chamadas de símbolos de Christoffel, tais que são os componentes da conexão $\nabla$ ou seja:

$$
\nabla_{X_{i}} X_{j}(p)=\sum_{k=1}^{m} \Gamma_{i j}^{k}(p) X_{k} .
$$

$\operatorname{Dados} X, Y \in \mathfrak{X}(M)$, pode-se tomar $\left.X\right|_{U}=\sum_{i=1}^{m} a_{i} X_{i}$ e $\left.Y\right|_{U}=\sum_{j=1}^{m} b_{j} X_{j}$, com $a_{i}, b_{j} \in \mathcal{D}(M)$, e aplicando-se as propriedades de linearidade (1) e (3) da conexão afim $\nabla$ obtem-se que:

$$
\nabla_{X} Y=\sum_{i=1}^{m} a_{i} \nabla_{X_{i}} \sum_{j=1}^{m} b_{j} X_{j}
$$

e por (2) e (4):

$$
\begin{aligned}
\nabla_{X} Y & =\sum_{i=1}^{m} a_{i}\left(\sum_{j=1}^{m} X_{i}\left[b_{j}\right] X_{j}+b_{j} \nabla_{X_{i}} X_{j}\right) \\
& =\sum_{i=1}^{m} a_{i}\left(\sum_{k=1}^{m} X_{i}\left[b_{k}\right] X_{k}+\sum_{j=1}^{m} b_{j} \sum_{k=1}^{m} \Gamma_{i j}^{k} X_{k}\right) \\
& =\sum_{k=1}^{m}\left(\sum_{i=1}^{m} a_{i} X_{i}\left[b_{k}\right]+\sum_{i, j=1}^{m} a_{i} b_{j} \Gamma_{i j}^{k}\right) X_{k} .
\end{aligned}
$$

Portanto $\nabla_{X} Y$ é dado em coordenadas pela seguinte expressão:

$$
\nabla_{X} Y(p)=\sum_{k=1}^{m}\left(X_{p}\left[b_{k}\right]+\sum_{i, j=1}^{m} a_{i}(p) b_{j}(p) \Gamma_{i j}^{k}(p)\right) X_{k}(p)
$$

Assim como se define a "derivada direcional" $X_{p}[f]$ de uma função $f$ com relação ao vetor $X_{p}$, o que se faz agora é definir uma "taxa de variação" do campo $Y$ em $p$ na direção de $X_{p}$, que é medida por $\nabla_{X} Y(p)$. Conforme mostra a expressão de $\nabla_{X} Y(p)$, esta também é uma noção local: só depende da conexão $\nabla$, do valor de $X$ em $p$ e dos valores de $Y$ ao longo de qualquer curva diferenciável que passa por $p$ e tem vetor tangente $X_{p}$ em $p$. Portanto $\nabla_{X} Y(p)=\nabla_{X_{p}} Y$.

Decorre deste fato a seguinte proposição: 
Proposição 1.8. Seja $M$ com uma conexão afim $\nabla$. Então existe uma única lei $\left(\frac{D}{d t}\right)$ que associa a um campo $V=V(t)$ ao longo de uma curva $\alpha: I \subset \mathbb{R} \rightarrow M$ um outro campo $\frac{D V}{d t}$ ao longo de $\alpha$, denominado derivada covariante de $V$ e tal que:

(a) $\frac{D}{d t}(V+W)=\frac{D V}{d t}+\frac{D W}{d t}$

(b) $\frac{D}{d t}(f V)=\frac{d f}{d t} V+f \frac{D V}{d t}$, com $f \in \mathcal{D}(M) e \frac{d f}{d t}=\frac{d}{d t} f(\alpha(t))$

(c) Se $V$ é a restrição de um campo $Y \in \mathfrak{X}(M)$ à $\alpha$, ou seja, $V(t)=\left.Y\right|_{\alpha(t)}$ então $\frac{D V}{d t}=\nabla_{\dot{\alpha}(t)} Y$.

PROVA. Suponha que exista uma tal lei satisfazendo (a), (b) e (c).

Seja $(U, x)$ um sistema de coordenadas com $\alpha(I) \cap U \neq \emptyset \mathrm{e} x_{i}(t)=\left(x_{i} \circ \alpha\right)(t)$, $\left\{X_{j}=\frac{\partial}{\partial x_{j}}\right\}$ base de $T_{p} M \operatorname{com} X_{j}=X_{j}(\alpha(t))$. Então $\left.V\right|_{U}=\sum_{j=1}^{m} v_{j}(t) X_{j}$ e

$$
\begin{aligned}
\frac{D V}{d t} & =\frac{D}{d t}\left(\sum_{j=1}^{m} v_{j} X_{j}\right) \\
& \stackrel{(a)}{=} \sum_{j=1}^{m} \frac{D}{d t}\left(v_{j} X_{j}\right) \stackrel{(b)}{=} \sum_{j=1}^{m} \frac{d v_{j}}{d t} X_{j}+v_{j} \frac{D X_{j}}{d t} \stackrel{(c)}{=} \sum_{j=1}^{m} \frac{d v_{j}}{d t} X_{j}+v_{j} \nabla_{\dot{\alpha}(t)} X_{j} .
\end{aligned}
$$

Como $\dot{\alpha}(t)=\sum_{i=1}^{m} \frac{d x_{i}}{d t} X_{i}$ e pelas propriedades (1) e (3) da conexão $\nabla$ obtem-se:

$$
\begin{aligned}
\frac{D V}{d t} & =\sum_{j=1}^{m} \frac{d v_{j}}{d t} X_{j}+v_{j} \sum_{i=1}^{m} \frac{d x_{i}}{d t} \nabla_{X_{i}} X_{j} \\
& =\sum_{k=1}^{m}\left(\frac{d v_{k}}{d t}+\sum_{i, j=1}^{m} v_{j} \frac{d x_{i}}{d t} \Gamma_{i j}^{k}\right) X_{k}
\end{aligned}
$$

Portanto esta é a lei da derivada covariante, cuja unicidade fica garantida pela própria expressão dada, e satisfaz (a), (b) e (c) pois foi deduzida a partir delas.

DEFINIÇÃO 1.9. Um campo $V \in \mathfrak{X}(M)$ ao longo de uma curva $\alpha: I \rightarrow M e ́$ dito paralelo, ou constante, na conexão $\nabla$ quando $\frac{D V}{d t}=0$ para todo $t \in I$.

PROPOSIÇão 1.10. Seja M com conexão afim $\nabla$. Considere $\alpha: I \rightarrow$ M uma curva diferenciável e $V_{0}$ um vetor tangente a $M$ em $\alpha\left(t_{0}\right), t_{0} \in I$. Então existe um campo $V$ paralelo ao longo de $\alpha$ tal que $V\left(t_{0}\right)=V_{0}$.

Para cada $t \in I$, diz-se que $V(t)$ é o transporte paralelo de $V_{0}$ ao longo de $\alpha$.

Prova. Suponha inicialmente que $\alpha(I) \subset U$ onde $(U, x)$ é um sistema de coordenadas de $M ; \alpha(t)=\left(x_{1}(t), \cdots, x_{m}(t)\right)$.

Seja $V_{0}=\sum_{j=1}^{m} v_{0}^{j} X_{j}\left(\alpha\left(t_{0}\right)\right)$ e suponha que exista $V(t)=\sum_{j=1}^{m} v_{j}(t) X_{j}(\alpha(t))$ 
paralelo ao longo de $\alpha \operatorname{com} V\left(t_{0}\right)=V_{0}$. Então $V$ satisfaz:

$$
\left\{\begin{array}{l}
0=\frac{D V}{d t}=\sum_{k=1}^{m}\left(\frac{d v_{k}}{d t}+\sum_{i, j=1}^{m} v_{j} \frac{d x_{i}}{d t} \Gamma_{i j}^{k}\right) X_{k} \\
V\left(t_{0}\right)=V_{0}
\end{array}\right.
$$

Ou ainda:

$$
\left\{\begin{array}{l}
\frac{d v_{k}}{d t}=-\sum_{i, j=1}^{m} v_{j} \frac{d x_{i}}{d t} \Gamma_{i j}^{k} \quad k=1, \cdots, m . \\
v_{k}\left(t_{0}\right)=v_{0}^{k}
\end{array}\right.
$$

Como este é um sistema linear de equações diferenciais de primeira ordem com condições iniciais, pelo Teorema de existência e unicidade das E.D.O. a solução $v_{k}(t)$ existe para todo $t \in I$ e é única. Portanto, basta tomar $V(t)=\sum_{j=1}^{m} v_{j}(t) X_{j}(\alpha(t))$.

Se $\alpha(I)$ não estiver contida num único sistema de coordenadas, como $\alpha\left(\left[t_{0}, t\right]\right)$, $t \in I$, é compacto em $M$, pode ser coberto por um conjunto finito de vizinhanças coordenadas e nas intersecções não vazias a unicidade da solução está garantida.

DEFINIÇÃo 1.11. Uma curva $\gamma: I \rightarrow M$ é uma geodésica, na conexão $\nabla$, se $\dot{\gamma}(t)$ é paralelo ao longo de $\gamma$, isto é $\frac{D}{d t} \dot{\gamma}(t)=\nabla_{\dot{\gamma}(t)} \dot{\gamma}(t)=0$ para todo $t \in I$.

Proposição 1.12. Sejam $p \in M$ e $v \in T_{p} M$. Para todo $t_{0} \in \mathbb{R}$, existe $\delta>0$ e uma única curva $\gamma$ definida em $] t_{0}-\delta, t_{0}+\delta\left[\right.$ tal que $\gamma\left(t_{0}\right)=p, \dot{\gamma}\left(t_{0}\right)=v$ e $\gamma e ́$ uma geodésica.

Prova. Se $(U, x)$ é um sistema de coordenadas em $p$, pela Proposição 1.10

$$
\dot{\gamma}(t)=\sum_{k=1}^{m} \frac{d x_{k}}{d t}(t) \frac{\partial}{\partial x_{k}}
$$

é o transporte paralelo de $\dot{\gamma}\left(t_{0}\right)=v$ em cada $t$ e segue daí imediatamente a unicidade local da geodésica $\gamma(t)$, dada pela equação diferencial $\nabla_{\dot{\gamma}} \dot{\gamma} \equiv 0$ :

$$
\frac{d^{2} x_{k}}{d t^{2}}+\sum_{i, j=1}^{m} \frac{d x_{i}}{d t} \frac{d x_{j}}{d t} \Gamma_{i j}^{k}=0
$$

com condições iniciais:

$$
x_{k}\left(t_{0}\right)=x_{k}(p) \text { e } \frac{d x_{k}}{d t}\left(t_{0}\right)=v_{k} \text { para cada } k=1, \cdots, m .
$$

DEFINIÇÃo 1.13. Uma curva integral de um campo $X \in \mathfrak{X}(M)$ é uma curva diferenciável $\alpha: I \rightarrow M$ tal que $\dot{\alpha}(t)=X(\alpha(t))$ para todo t no domínio de $\alpha$.

DEFINIÇÃo 1.14. Uma conexão afim $\nabla$ é dita simétrica se satisfaz

$$
[X, Y]=\nabla_{X} Y-\nabla_{Y} X
$$

para todo $X, Y \in \mathfrak{X}(M)$. $[X, Y]$ é o colchete de Lie de $X$ e $Y$ (Definição 1.1). 
Os símbolos de Christoffel são simétricos em uma conexão simétrica (assim denominada por isto) porque em campos coordenados, pelo Teorema de Schwarz,

$$
\left[X_{i}, X_{j}\right]=0 \Longrightarrow \nabla_{X_{i}} X_{j}=\nabla_{X_{j}} X_{i} \Longrightarrow \Gamma_{i j}^{k}=\Gamma_{j i}^{k} \text {. }
$$

DEFINIÇão 1.15. Uma conexão afim $\nabla$ é dita compatível com a métrica em uma variedade Riemanniana $(M, g)$ quando

$$
X g(Y, Z)=g\left(\nabla_{X} Y, Z\right)+g\left(Y, \nabla_{X} Z\right)
$$

para todo $X, Y, Z \in \mathfrak{X}(M)$.

COROLÁRIO 1.16. Seja $\nabla$ uma conexão afim compativel com a métrica em $(M, g)$. A expressão (1.2) em termos da derivada covariante é dada por:

$$
\frac{d}{d t} g(V(t), W(t))=g\left(\frac{D V}{d t}, W(t)\right)+g\left(V(t), \frac{D W}{d t}\right)
$$

para todo $V, W \in \mathfrak{X}(M)$.

Prova. Como $\nabla_{X} Y(p)=\nabla_{X_{P}} Y$ a expressão (1.2) em $p$ é calculada por:

$$
X_{p} g(Y, Z)=g\left(\nabla_{X_{p}} Y, Z(p)\right)+g\left(Y(p), \nabla_{X_{p}} Z\right) .
$$

Considere $\alpha: I \rightarrow M$ uma curva integral de $X$ (Definição 1.13) e tome $Y$ e $Z$ restritos à curva $\alpha:\left.Y\right|_{\alpha(t)}=Y(t)$ e $\left.Z\right|_{\alpha(t)}=Z(t)$. Afirma-se que:

$$
\frac{d}{d t} g(Y(t), Z(t))=g\left(\nabla_{\dot{\alpha}(t)} Y, Z(t)\right)+g\left(Y(t), \nabla_{\dot{\alpha}(t)} Z\right) .
$$

De fato, pois se $f(x)=g(Y(x), Z(x)), x \in M$, então

$$
\frac{d}{d t} g(Y(t), Z(t))=\frac{d}{d t} f(\alpha(t))=d f_{\alpha(t)}(\dot{\alpha}(t))=X_{\alpha(t)}[f]
$$

e por (1.4)

$$
\frac{d}{d t} g(Y(t), Z(t))=g\left(\nabla_{\dot{\alpha}(t)} Y, Z(t)\right)+g\left(Y(t), \nabla_{\dot{\alpha}(t)} Z\right) .
$$

Chamando $\left.Y\right|_{\alpha(t)}=V(t)$ e $\left.Z\right|_{\alpha(t)}=W(t)$ em (1.5) e pela propriedade (c) da derivada covariante (Proposição 1.8), obtem-se a expressão (1.3) que caracteriza a compatibilidade da conexão com a métrica, em termos da derivada covariante:

$$
\frac{d}{d t} g(V(t), W(t))=g\left(\frac{D V}{d t}, W(t)\right)+g\left(V(t), \frac{D W}{d t}\right) .
$$

PROPOSIÇ̃̃o 1.17. Seja $(M, g)$ com conexão afim $\nabla$. Então $\nabla$ é compatível com a métrica se e somente se para toda curva diferenciável $\alpha: I \rightarrow M$ e para todo par de campos $V(t), W(t)$, paralelos ao longo de $\alpha$, tem-se que a aplicação $t \rightarrow g(V(t), W(t))$ é constante. 
Prova. Como $V(t)$ e $W(t)$ são paralelos ao longo de $\alpha$, a expressão (1.3) anula-se: $\frac{d}{d t} g(V(t), W(t)) \equiv 0$ e portanto a aplicação é constante.

Tome agora uma base ortonormal $\left\{E_{1}, \cdots, E_{m}\right\}$ de $T_{\alpha\left(t_{0}\right)} M$, para algum $t_{0} \in I$, em relação à métrica $g$ de $M$, isto é, $g\left(E_{i}, E_{j}\right)=\delta_{i j}$ e considere seu transporte paralelo ao longo de $\alpha$, conforme a Proposição 1.10.

Como a aplicação $t \rightarrow g(V(t), W(t))$ é constante, este referencial é ortonormal também ao longo de $\alpha$, ou seja, $\left\{E_{1}(t), \cdots, E_{m}(t)\right\}$ é uma base ortonormal de $T_{\alpha(t)} M$ em cada $\alpha(t)$.

Todo par de campos $V(t), W(t)$ ao longo de $\alpha$ pode ser dado por:

$V(t)=\sum_{j=1}^{m} v_{j}(t) E_{j}(t)$ e $W(t)=\sum_{k=1}^{m} w_{k}(t) E_{k}(t)$.

Daí, $\frac{d}{d t} g(V(t), W(t))=\frac{d}{d t}\left(\sum_{j=1}^{m} v_{j}(t) w_{j}(t)\right)=\sum_{j=1}^{m} \frac{d v_{j}}{d t} w_{j}+v_{j} \frac{d w_{j}}{d t}$.

Mas, pelas propriedades (a) e (b) da derivada covariante, tem-se:

$\frac{D V}{d t}=\frac{D}{d t}\left(\sum_{j=1}^{m} v_{j}(t) E_{j}(t)\right)=\sum_{j=1}^{m} \frac{d v_{j}}{d t} E_{j}$, pois $E_{j}$ é paralelo. Segue que:

$g\left(\frac{D V}{d t}, W(t)\right)=g\left(\sum_{j=1}^{m} \frac{d v_{j}}{d t} E_{j}, \sum_{k=1}^{m} w_{k} E_{k}\right)=\sum_{j=1}^{m} \frac{d v_{j}}{d t} w_{j}$.

Portanto, $\frac{d}{d t} g(V(t), W(t))=g\left(\frac{D V}{d t}, W(t)\right)+g\left(V(t), \frac{D W}{d t}\right)$, ou seja, vale (1.3).

Para $\nabla$ ser compatível com a métrica é preciso mostrar que vale a expressão (1.2) para quaisquer campos em $\mathfrak{X}(M)$. Para isto, considere $X, Y, Z \in \mathfrak{X}(M), p \in M$ e $\gamma:]-\epsilon, \epsilon\left[\rightarrow M\right.$ a única curva em $M \operatorname{com} \gamma(0)=p$ e $\dot{\gamma}(0)=X_{p}$. Então

$$
X_{p} g(Y, Z)=\dot{\gamma}(0)[g(Y, Z)]=\left.\frac{d}{d t}(g(Y, Z) \circ \gamma(t))\right|_{t=0}=\left.\frac{d}{d t} g(Y(t), Z(t))\right|_{t=0}
$$

e por (1.3)

$$
\begin{aligned}
X_{p} g(Y, Z) & =\left.g\left(\frac{D Y}{d t}, Z(t)\right)\right|_{t=0}+\left.g\left(Y(t), \frac{D Z}{d t}\right)\right|_{t=0} \\
& =g\left(\nabla_{X_{p}} Y, Z(p)\right)+g\left(Y(p), \nabla_{X_{p}} Z\right) .
\end{aligned}
$$

E portanto, como $p$ é arbitrário,

$$
X g(Y, Z)=g\left(\nabla_{X} Y, Z\right)+g\left(Y, \nabla_{X} Z\right) \text {. }
$$

TEOREMA 1.18. Teorema Fundamental da Geometria Riemanniana

Dada uma variedade Riemanniana $(M, g)$ existe uma única conexão afim $\nabla$ tal que:

(a) $\nabla$ é simétrica

(b) $\nabla$ é compatível com a métrica.

Uma tal conexão é chamada Riemanniana ou conexão Levi-Civita de g em M.

PRova. Seja $\nabla$ satisfazendo (a) e (b). 
Por (b) valem as seguintes expressões para todo $X, Y, Z \in \mathfrak{X}(M)$

$$
\begin{aligned}
& X g(Y, Z)=g\left(\nabla_{X} Y, Z\right)+g\left(Y, \nabla_{X} Z\right) \\
& Y g(X, Z)=g\left(\nabla_{Y} X, Z\right)+g\left(X, \nabla_{Y} Z\right) \\
& Z g(X, Y)=g\left(\nabla_{Z} X, Y\right)+g\left(X, \nabla_{Z} Y\right)
\end{aligned}
$$

e segue que:

$$
\begin{aligned}
& X g(Y, Z)+Y g(X, Z)-Z g(X, Y) \\
= & g\left(\nabla_{X} Y, Z\right)+g\left(Y, \nabla_{X} Z\right)-g\left(Y, \nabla_{Z} X\right) \\
+ & g\left(\nabla_{X} Y, Z\right)+g\left(Z, \nabla_{Y} X\right)-g\left(Z, \nabla_{X} Y\right) \\
+ & g\left(X, \nabla_{Y} Z\right)-g\left(X, \nabla_{Z} Y\right) .
\end{aligned}
$$

Por (a) e como $[Y, X]=-[X, Y]$ (Definição 1.1), tem-se:

$$
\begin{aligned}
& X g(Y, Z)+Y g(X, Z)-Z g(X, Y) \\
= & 2 g\left(\nabla_{X} Y, Z\right)+g(Y,[X, Z])-g(Z,[X, Y])+g(X,[Y, Z])
\end{aligned}
$$

Obtemos assim, a chamada Fórmula de Koszul:

$$
\begin{aligned}
& g\left(\nabla_{X} Y, Z\right)=\frac{1}{2} \quad\{X g(Y, Z)+Y g(X, Z)-Z g(X, Y) \\
& \text { - } g(X,[Y, Z])-g(Y,[X, Z])+g(Z,[X, Y])\}
\end{aligned}
$$

tal que $\nabla$ determinada por esta expressão é única e satisfaz (a) e (b).

A expressão (1.6) calculada nos campos coordenados fornece a expressão clássica dos símbolos de Christoffel em termos dos $g_{i j}$ da métrica ou seja, permite determinar a conexão Levi-Civita a partir da métrica. Como

$$
g\left(\nabla_{X_{i}} X_{j}, X_{l}\right)=g\left(\sum_{k} \Gamma_{i j}^{k} X_{k}, X_{l}\right)=\sum_{k} \Gamma_{i j}^{k} g_{k l}
$$

e por (1.6)

$$
g\left(\nabla_{X_{i}} X_{j}, X_{l}\right)=\frac{1}{2}\left\{X_{i}\left(g_{j l}\right)+X_{j}\left(g_{i l}\right)-X_{l}\left(g_{i j}\right)\right\}
$$

denotando por $\left(g^{s l}\right)$ a inversa da matriz $\left(g_{k l}\right)$ ou seja, $g^{s l} \cdot g_{k l}=\delta_{s, k}$, obtem-se:

$$
\Gamma_{i j}^{s}=\frac{1}{2} \sum_{l=1}^{m} g^{s l}\left\{\frac{\partial}{\partial x_{i}}\left(g_{j l}\right)+\frac{\partial}{\partial x_{j}}\left(g_{i l}\right)-\frac{\partial}{\partial x_{l}}\left(g_{i j}\right)\right\} .
$$

DEFINIÇÃo 1.19. A curvatura $R$ de uma variedade Riemanniana $(M, g)$ é uma aplicação que associa a cada par $(X, Y) \in \mathfrak{X}(M) \times \mathfrak{X}(M)$ uma aplicação $R(X, Y): \mathfrak{X}(M) \rightarrow \mathfrak{X}(M)$ dada por

$$
R(X, Y) Z=\nabla_{X} \nabla_{Y} Z-\nabla_{Y} \nabla_{X} Z-\nabla_{[X, Y]} Z
$$

onde $\nabla$ é a conexão Levi-Civita de $g$ em $M$.

As seguintes propriedades são satisfeitas para todo $X, Y, Z, T, X_{1}, X_{2}, Y_{1}, Y_{2} \in \mathfrak{X}(M)$ e $f, f_{1}, f_{2} \in \mathcal{D}(M)$ 
(1) $R$ é um conceito local, intrínseco, pois depende apenas da métrica $g$ em $M$. É um tensor do tipo $(0,2)$, assim como a métrica $g$ ou seja, é bilinear em $\mathfrak{X}(M)$ :

$$
\begin{aligned}
& R\left(f_{1} X_{1}+f_{2} X_{2}, Y\right)=f_{1} R\left(X_{1}, Y\right)+f_{2} R\left(X_{2}, Y\right) \\
& R\left(X, f_{1} Y_{1}+f_{2} Y_{2}\right)=f_{1} R\left(X, Y_{1}\right)+f_{2} R\left(X, Y_{2}\right) ;
\end{aligned}
$$

(2) O operador curvatura $R(X, Y)$ é linear em $\mathfrak{X}(M)$ :

$R(X, Y)(Z+T)=R(X, Y) Z+R(X, Y) T$

$R(X, Y) f Z=f R(X, Y) Z$

(3) Para o tensor curvatura $R(X, Y) Z$, do tipo $(0,3)$, valem:

(a) $R(X, Y) Z+R(Y, Z) X+R(Z, X) Y=0$

(b) $g(R(X, Y) Z, T)=-g(R(X, Y) T, Z)$

(c) $g(R(X, Y) Z, T)=-g(R(Y, X) Z, T)$

(d) $g(R(X, Y) Z, T)=g(R(Z, T) X, Y)$.

$g(R(X, Y) Z, T)$ é chamado de o tensor curvatura Riemanniana do tipo $(0,4)$ ou seja, é uma aplicação 4-linear que a cada $(X, Y, Z, T)$ associa $g(R(X, Y) Z, T)$, também denotado por $R(X, Y, Z, T)$.

DEFINIÇÃo 1.20. Dados $p \in M$ e $\sigma \subset T_{p} M$ um subespaço bidimensional de $T_{p} M$, o número real $K_{p}(\sigma)$ é a curvatura seccional de $\sigma$ em $p$ e é dado por:

$$
K_{p}(\sigma)=\frac{g(R(v, w) w, v)}{\|v \times w\|^{2}}
$$

onde $\{v, w\}$ é uma base de $\sigma$ e $\|v \times w\|^{2}=\|v\|^{2}\|w\|^{2}-g(v, w)^{2}$.

$$
K_{p}(\sigma) \text { está bem definida pois independe da base escolhida de } \sigma \text {. }
$$

\section{Gradiente e Hessiano de funções definidas em variedades Riemannianas}

DEFINIÇão 1.21. Dada $(M, g)$ uma variedade Riemanniana, sejam $p \in M e$ $F: M \rightarrow \mathbb{R}$ uma função de classe $C^{1} . O$ gradiente de $F \operatorname{em} p, \nabla F_{p}$, denota o único vetor de $T_{p} M$ tal que

$$
g\left(\nabla F_{p}, v\right)=\mathrm{d} F_{p}(v)
$$

onde $\mathrm{d} F_{p}(v)$ é a diferencial de $F$ em $p$ na direção de $v \in T_{p} M$.

DEFINIÇÃo 1.22. Se $F: M \rightarrow \mathbb{R}$ é duas vezes diferenciável em $p \in M$ entãoo hessiano de $F$ em $p$ é uma forma bilinear simétrica em $T_{p} M$ dada por

$$
\text { Hess } F(p)(v, w)=g\left(\nabla_{v} \nabla F(p), w\right)
$$

para todo $v, w \in T_{p} M$, onde $\nabla$ é a conexão Levi-Civita de g e $\nabla F$ denota o campo gradiente de F em $M$. 
Lembremos que: Dada uma forma bilinear $H$ em um espaço vetorial $V$, a função $Q: V \rightarrow \mathbb{R}$ definida por $Q(v)=H(v, v)$ é a forma quadrática associada à forma bilinear $H$ em $V$. Reciprocamente, dada uma forma quadrática $Q$ em $V$ existe uma única forma bilinear simétrica $H$ tal que $H(v, v)=Q(v)$.

Em geral é mais fácil lidar com $Q$ do que com $H$, e não há perda de informação com esta troca pois $H$ pode ser obtida novamente pela identidade de polarização:

$$
H(v, w)=\frac{1}{2}[Q(v+w)-Q(v)-Q(w)] .
$$

LEMA 1.23. Sejam $p \in M$ e $v \in T_{p} M$. Considere $\left.\gamma:\right]-\delta, \delta[\rightarrow M$ a única geodésica em $M$ tal que $\gamma(0)=p$ e $\dot{\gamma}(0)=v$. Então o valor da forma quadrática Hess $F(p)(v, v)$ é calculado por:

$$
\text { Hess } F(p)(v, v)=\left.\frac{d^{2}}{d t^{2}}(F \circ \gamma)(t)\right|_{t=0} .
$$

Prova. Pela Definição 1.22 do hessiano de $F \operatorname{em} p=\gamma(0)$, pela compatibilidade da conexão com a métrica (Corolário 1.16) e como, pela Definição 1.11 de geodésica, $\nabla_{\dot{\gamma}} \dot{\gamma} \equiv 0$, obtem-se que:

$$
\text { Hess } F(p)(v, v)=\left.g\left(\nabla_{\dot{\gamma}(t)} \nabla F(\gamma(t)), \dot{\gamma}(t)\right)\right|_{t=0}=\left.\frac{d}{d t} g(\nabla F(\gamma(t)), \dot{\gamma}(t))\right|_{t=0} .
$$

Portanto, pela Definição 1.21 do gradiente de $F$ em $p$, segue que:

$$
\operatorname{Hess} F(p)(v, v)=\left.\frac{d}{d t}\left[\mathrm{~d} F_{\gamma(t)}(\dot{\gamma}(t))\right]\right|_{t=0}=\left.\frac{d^{2}}{d t^{2}}(F \circ \gamma)(t)\right|_{t=0} .
$$

DEFInIÇão 1.24. Seja $F: M \rightarrow \mathbb{R}$ de classe $C^{1}$. Diz-se que $p_{0} \in M$ é um ponto crítico de $F$ se $\mathrm{d} F_{p_{0}} \equiv 0$.

$F\left(p_{0}\right)$ é chamado um valor crítico de $F$. Se $c \in \mathbb{R}$ não é um valor crítico de $F$ e $F^{c}=\{q \in M: F(q) \leq c\}$ então, pelo Teorema da Função Implícita $F^{c}$ é uma subvariedade regular com bordo. O bordo $F^{-1}(c)$ é uma subvariedade regular de $M$.

O hessiano de $F$ em um ponto crítico $p_{0}$ não depende da métrica $g$ em $M$. De fato, se $v, w \in T_{p_{0}} M$ então $v$ e $w$ possuem extensões aos campos vetoriais $\tilde{v}$ e $\tilde{w}$ de $T M$ tal que $\tilde{v}_{p_{0}}=v$ e $\tilde{w}_{p_{0}}=w$. Definindo-se

$$
\operatorname{Hess} F\left(p_{0}\right)(\tilde{v}, \tilde{w})=v[\tilde{w}[F]]
$$

segue que esta é uma forma simétrica pois, como $p_{0}$ é crítico e pela Definição 1.1,

$$
0=[\tilde{v}, \tilde{w}]\left(p_{0}\right)[F]=v[\tilde{w}[F]]-w[\tilde{v}[F]],
$$

e logo $v[\tilde{w}[F]]=w[\tilde{v}[F]]$. E está bem definida pois $\operatorname{Hess} F\left(p_{0}\right)(\tilde{v}, \tilde{w})=v[\tilde{w}[F]]$ não depende da extensão $\tilde{v}$ tomada e, como $\operatorname{Hess} F\left(p_{0}\right)(\tilde{v}, \tilde{w})=v[\tilde{w}[F]]=w[\tilde{v}[F]]$, 
também não depende da extensão $\tilde{w}$.

Como $X g(\nabla F, Y)=g\left(\nabla_{X} \nabla F, Y\right)+g\left(\nabla F, \nabla_{X} Y\right)$ (Definição 1.15) e pela definição de $\nabla F, g(\nabla F, Y)=Y[F]$, o hessiano de $F$ é dado também por:

Hess $F(X, Y)=X[Y[F]]-\nabla_{X} Y[F]$.

Daí, em um ponto crítico $p_{0}$, tem-se que $\operatorname{Hess} F\left(p_{0}\right)(X, Y)=X_{p_{0}}[Y[F]]$.

Seja $(U, x)$ um sistema de coordenadas local em $p$ e $\left(\frac{\partial}{\partial x_{i}}\right)$ uma base de $T_{p} M$. Tome $v=X_{p}=\sum_{i=1}^{m} a_{i} \frac{\partial}{\partial x_{i}}$ e $w=Y_{p}=\sum_{j=1}^{m} b_{j} \frac{\partial}{\partial x_{j}}$, $\operatorname{com} a_{i}, b_{j} \in \mathcal{D}(p)$.

Como Hess $F(p)(X, Y)=X_{p}[Y[F]]-\nabla_{X} Y(p)[F]$,

$$
X_{p}[Y[F]]=\left(\sum_{i=1}^{m} a_{i} \frac{\partial}{\partial x_{i}}\right)\left[\sum_{j=1}^{m} b_{j} \frac{\partial F}{\partial x_{j}}\right]=\sum_{i, j=1}^{m} a_{i}\left(\frac{\partial b_{j}}{\partial x_{i}} \frac{\partial F}{\partial x_{j}}+b_{j} \frac{\partial^{2} F}{\partial x_{i} \partial x_{j}}\right)
$$

e pela Definição 1.7

$$
\begin{aligned}
& \nabla_{X} Y(p)[F]=\sum_{k=1}^{m}\left(\sum_{i=1}^{m} a_{i} \frac{\partial b_{k}}{\partial x_{i}}+\sum_{i, j=1}^{m} a_{i} b_{j} \Gamma_{i j}^{k}\right) \frac{\partial F}{\partial x_{k}}, \\
& \text { Hess } F(p)(v, w)=\sum_{i, j, k=1}^{m} a_{i} b_{k}\left(\frac{\partial^{2} F}{\partial x_{i} \partial x_{k}}-\Gamma_{i k}^{j} \frac{\partial F}{\partial x_{j}}\right) .
\end{aligned}
$$

Logo, as componentes locais do hessiano de $F$ em $p$ são dadas por:

$$
f_{i j}=\frac{\partial^{2} F}{\partial x_{i} \partial x_{j}}-\Gamma_{i j}^{k} \frac{\partial F}{\partial x_{k}} .
$$

E, como em um ponto crítico $p_{0}$, tem-se em coordenadas locais que $\frac{\partial F}{\partial x_{i}}\left(p_{0}\right)=0$ para todo $i=1, \cdots, m$, o hessiano de $F$ é representado pela clássica matriz Hessiana:

$$
f_{i j}=\frac{\partial^{2} F}{\partial x_{i} \partial x_{j}} .
$$

Para introduzir a noção de nulidade de uma forma bilinear $H$, é interessante retomar o conceito, com ela relacionado, de índice de $H$ dado na Observação (1.3):

DEFINIÇÃo 1.25. $O$ índice $\lambda$ de uma forma bilinear $H$ em um espaço vetorial $V$ é definido como o máximo das dimensões dos subespaços $W$ de $V$ nos quais $\left.H\right|_{W}$ é definida negativa.

A nulidade de $H$ é a dimensão do espaço-nulo de $H$ isto é, do subespaço de $V$ constituído por todo $v$ em $V$ tal que $H(v, w)=0$ para todo $w \in V$.

O índice do Hess $F(p)$ é chamado simplesmente de indice de F em $p$, denota-se Hess $F(p)(v, w)$ por $I[v, w]$ e diz-se que o espaço-nulo de Hess $F(p)$ é o núcleo de $I: \operatorname{Nuc}(I)=\left\{v \in T_{p} M: I[v, w]=0\right.$ para todo $\left.w \in T_{p} M\right\}$. 
Como $g$ é não-degenerada, a forma bilinear Hess $F(p)$ é realizada por um funcional linear em $T_{p} M$, o operador hessiano de $F$ em $p$, hess $F(p)$, tal que:

$$
\text { hess } F(p)_{v}(w)=g\left(\text { hess } F(p)_{v}, w\right)=\operatorname{Hess} F(p)(v, w) \text {. }
$$

Então o espaço-nulo de Hess $F(p)$ coincide com o núcleo do operador hessiano:

Nuc $(I)=\left\{v \in T_{p} M:\right.$ hess $F(p)_{v}(w)=0$ para todo $\left.w \in T_{p} M\right\}$.

DEFINIÇão 1.26. Um ponto crítico $p_{0}$ de $F$, de classe $C^{2}$, é dito não-degenerado se o operador hessiano hess $F\left(p_{0}\right)$ é invertível ou seja, se Hess $F\left(p_{0}\right)$ é uma forma não-degenerada. Ou equivalentemente: $O$ ponto $p_{0}$ é um ponto crítico não-degenerado de $F$ se Hess $F\left(p_{0}\right)$ em $T_{p_{0}} M$ tem nulidade igual a zero ou seja, Nuc $(I)=\{0\}$.

O comportamento de $F$ em uma vizinhança aberta de $p_{0}$ pode ser completamente descrito a partir do índice $\lambda$, como mostra o seguinte lema:

\section{LEMA 1.27. Lema de Morse}

Seja $p_{0}$ um ponto crítico não-degenerado de $F: M \rightarrow \mathbb{R}$, de classe $C^{2}$. Então existe um sistema de coordenadas local de $M$ em $p_{0},(U, \varphi=x) \operatorname{com} x_{i}\left(p_{0}\right)=0$ para todo i e tal que a identidade

$$
F=F\left(p_{0}\right)-x_{1}^{2}-\cdots-x_{\lambda}^{2}+x_{\lambda+1}^{2}+\cdots+x_{m}^{2}
$$

ocorre em $U$ e onde $\lambda$ é $o$ índice de $F$ em $p_{0}$.

([12, Lema 2.2])

Lembremos também de uma bem conhecida propriedade do hessiano:

Proposição 1.28. Seja $f: U \subset \mathbb{R}^{m} \rightarrow \mathbb{R}, C^{2}$. Se $x_{0} \in U$ é um ponto crítico de $f$ e Hess $f\left(x_{0}\right)$ é definido positivo então $x_{0}$ é um ponto de mínimo local de $f$. Reciprocamente, se $x_{0}$ é um ponto de mínimo local então Hess $f\left(x_{0}\right)$ é semidefinido positivo.

([19, Capítulo 1 - Teorema 8.3])

Por esta propriedade, se $p_{0}$ é um ponto crítico não-degenerado de $F$ isto é, Nuc $(I)=\{0\}$, e se $\lambda=0$ então $p_{0}$ é um ponto de mínimo local de $F$.

\section{Segunda variação da Energia e conjugação ao longo de geodésicas}

3.1. Vizinhanças normais. Seja $\gamma: I \subset \mathbb{R} \rightarrow M$ uma geodésica em $M$. Para $[a, b] \subset I,\left.\gamma\right|_{[a, b]}$ é chamada de um segmento de geodésica ligando $p=\gamma(a)$ a $q=\gamma(b)$ e denotado por $\gamma_{p q}$. Por abuso de linguagem, muitas vezes chamamos de geodésica à imagem $\gamma([a, b]) \equiv \gamma_{p q}$.

Como $\nabla$ é a conexão Levi-Civita de $g$ em $M$ e $\dot{\gamma}(t)$ é um campo paralelo, segue imediatamente pela Proposição 1.17 que $g(\dot{\gamma}(t), \dot{\gamma}(t))$ é constante $(=c)$ para todo $t \in I$. Daí, a função comprimento de arco de uma geodésica é dada por:

$$
s(t)=\int_{t_{0}}^{t}\|\dot{\gamma}(r)\| d r=\sqrt{c}\left(t-t_{0}\right) .
$$


E portanto o parâmetro de uma geodésica é sempre proporcional ao comprimento de arco. Decorre deste fato o seguinte

LEMA 1.29. Se $\gamma:[a, b] \rightarrow M$ é um segmento geodésico ligando $p$ a $q$ em $M$ então existe $\sigma:[c, d] \rightarrow M$ uma reparametrização de $\gamma$ dada por $\sigma=\gamma \circ \phi$ onde $\phi:[c, d] \rightarrow[a, b]$ é um isomorfismo linear, com $c, d \in \mathbb{R} e[a, b] \subset \phi([c, d])$, tal que $\sigma$ também é um segmento geodésico ligando $p$ a q em $M$.

Prova. Como $\sigma(t)=\gamma(\phi(t))=\gamma(s) \Longrightarrow \dot{\sigma}(t)=\dot{\gamma}(s) \phi^{\prime}(t)$ e pela propriedade (b) da derivada covariante (Proposição 1.8), obtem-se que:

$$
\begin{aligned}
\frac{D}{d t} \dot{\sigma}(t) & =\frac{D}{d t} \dot{\gamma}(s)\left(\phi^{\prime}(t)\right)^{2}+\dot{\gamma}(s) \frac{D}{d t} \phi^{\prime}(t) \\
& =\dot{\gamma}(s) \phi^{\prime \prime}(t)=0
\end{aligned}
$$

pois $\phi$ é linear. E portanto, por definição, $\sigma$ é um segmento de geodésica.

Denote por $\gamma(t, q, v)$ a única geodésica que no instante $t=0$ passa por $q$ com velocidade $\dot{\gamma}(0, q, v)=v$.

LEMA 1.30. Lema da Homogeneidade.

Se a geodésica $\gamma(t, q, v)$ está definida em $]-\delta, \delta[$ então, para todo $a \in \mathbb{R}, a$ geodésica $\gamma(t, q, a v)$ está definida em $]-\frac{\delta}{a}, \frac{\delta}{a}[$ e $\gamma(t, q, a v)=\gamma(a t, q, v)$.

ProvA. Seja $\sigma:]-\frac{\delta}{a}, \frac{\delta}{a}[\rightarrow M$ uma reparametrização de $\gamma$ dada por $\sigma(t)=(\gamma \circ \phi)(t)=\gamma(a t, q, v)$ onde $\phi:]-\frac{\delta}{a}, \frac{\delta}{a}[\rightarrow]-\delta, \delta[$ é definido por

$\phi(t)=a t=s$. Pelo Lema $1.29 \sigma$ é uma geodésica tal que

$$
\dot{\sigma}(t)=\phi^{\prime}(t) \dot{\gamma}(s, q, v)=a \dot{\gamma}(s, q, v),
$$

e logo, satisfaz as condições iniciais: $\sigma(0)=q$ e $\dot{\sigma}(0)=a \dot{\gamma}(0, q, v)=a v$.

Portanto $\sigma(t)=\gamma(t, q, a v)$ e pela unicidade local das geodésicas (Proposição 1.12):

$$
\gamma(a t, q, v)=\gamma(t, q, a v) \text {. }
$$

Assim, pode-se tornar o intervalo de definição de uma geodésica uniformemente grande em uma vizinhança aberta de todo $q \in M$, através da extensão das soluções da sua equação diferencial (Proposição 1.12). Diz-se que uma geodésica $\gamma: I \rightarrow M e ́$ maximal se o domínio $I$ for o maior possível ou seja, se $\bar{\gamma}: J \rightarrow M$ for um segmento geodésico e existe $t \in I \cap J$ tal que $\gamma(t)=\bar{\gamma}(t)$ e $\dot{\gamma}(t)=\dot{\bar{\gamma}}(t)$ então $J \subset I$.

DEFINIÇão 1.31. Sejam $p \in M, \epsilon>0, \mathcal{U}_{p}=\mathcal{B}(0, \epsilon)=\left\{v \in T_{p} M:\|v\|<\epsilon\right\}$. $A$ aplicação exponencial $\exp _{p}: \mathcal{U}_{p} \rightarrow M$ é definida por:

$$
\exp _{p}(v)=\gamma(1, p, v)
$$

onde $\gamma$ é a única geodésica em $M$ que parte de $p$ com velocidade inicial $v$. 
Pelo Lema da Homogeneidade, $\exp _{p}(v)=\gamma(1, p, v)=\gamma\left(a, p, \frac{v}{a}\right)$ e se $a=\|v\|$, $\exp _{p}(v)=\gamma\left(\|v\|, p, \frac{v}{\|v\|}\right)$. Assim, geometricamente, $\exp _{p}(v)$ é o ponto de $M$ obtido percorrendo-se um comprimento $\|v\|$ a partir de $p$ sobre a geodésica com velocidade inicial $\frac{v}{\|v\|}$ e logo, normalizada.

LEMA 1.32. Dado $q \in M$, existe um $\epsilon>0$ tal que exp $p_{q}: \mathcal{B}(0, \epsilon) \rightarrow M$ é um difeomorfismo de $\mathcal{B}(0, \epsilon) \subset T_{q} M$ sobre um aberto de $M$.

A imagem $\exp _{q} \mathcal{B}(0, \epsilon)$, denotada por $\mathcal{B}(q, \epsilon)$, é chamada de bola geodésica de centro $q$ e raio $\epsilon$.

Prova. Basta mostrar que a diferencial de $\exp _{q}$ na origem de $T_{q} M$, $\left(\operatorname{dexp}_{q}\right)_{0}: T_{0}\left(T_{q} M\right) \simeq T_{q} M \rightarrow T_{\exp _{q} 0} M=T_{q} M$ é um isomorfismo, pois daí, pelo Teorema da Função Inversa, $\exp _{q}$ é um difeomorfismo local de uma vizinhança aberta da origem de $T_{q} M$ sobre uma vizinhança aberta de $q$ em $M$.

Dado $v \in T_{q} M$, pela definição de $\exp _{q}$ e pelo Lema da Homogeneidade (1.30):

$$
\begin{gathered}
\exp _{q} t v=\gamma(1, q, t v)=\gamma(t, q, v) \\
\left.\Longrightarrow \frac{d}{d t}\left(\exp _{q} t v\right)\right|_{t=0}=\left.\frac{d}{d t}(\gamma(t, q, v))\right|_{t=0}=\dot{\gamma}(0)=v
\end{gathered}
$$

Logo, $\left(\operatorname{dex} p_{q}\right)_{0}(v)=v$ e portanto $\left(\operatorname{dex} p_{q}\right)_{0} \equiv \mathrm{Id}$, a aplicação identidade de $T_{q} M . \square$

TEOREMA 1.33. Para todo $p \in M$ existe uma vizinhança $W$ de $p$ e um número $\epsilon^{\prime}>0$, tais que, para cada $q \in W$, exp $p_{q}$ é um difeomorfismo em $\mathcal{B}\left(0, \epsilon^{\prime}\right) \subset T_{q} M e$ $W \subset \exp _{q}\left(\mathcal{B}\left(0, \epsilon^{\prime}\right)\right)$.

$O$ conjunto $W$ é dito uma vizinhança normal ${ }^{1}$ (de centro) em $p$.

Prova. Seja $\mathcal{U}$ uma vizinhança de $(p, 0)$ em $T M$ onde a aplicação exponencial esteja definida. Quer dizer: $\mathcal{U} \subset T U$ onde $(U, \varphi)$ é um sistema de coordenadas local de $M$ em $p$ e tal que existem $\delta$ e $V \subset U \operatorname{com} p \in V$,

$\mathcal{U}=\left\{(q, v) \in T M: q \in V\right.$ e $\left.v \in T_{q} M,\|v\|<\delta\right\}$. Logo $\exp _{q}$ está definida para todo $q \in V$ (Definição 1.31).

Defina $F: \mathcal{U} \rightarrow M \times M$ por $F(q, v)=\left(q, \exp _{q} v\right) \mathrm{e}$ considere em $F(p, 0)=(p, p) \in M \times M$ o sistema de coordenadas $(U \times U ;(\varphi, \varphi))$. Assim, a matriz jacobiana de $\mathrm{d} F$ em $(p, 0)$ é dada por $\left(\begin{array}{ll}I & I \\ 0 & I\end{array}\right)$ pois, pelo Lema 1.32, $\left(\operatorname{dex} p_{p}\right)_{0} \equiv \mathrm{I}$. Como d $F_{(p, 0)}$ é não-singular, pelo Teorema da Função Inversa, existe uma vizinhança aberta $\mathcal{U}^{\prime} \subset \mathcal{U}$ de $(p, 0)$ e uma vizinhança aberta $W^{\prime}$ de $(p, p)$ em $M \times M$ tal que $F: \mathcal{U}^{\prime} \rightarrow W^{\prime}$ é um difeomorfismo local.

Suponha $\mathcal{U}^{\prime}=\left\{(q, v) \in T M: q \in V^{\prime}\right.$ e $\left.v \in T_{q} M,\|v\|<\epsilon^{\prime}\right\}$, onde, é claro, $V^{\prime} \subset V$ e $p \in V^{\prime}$. Escolha agora $W$, uma vizinhança aberta de $p$, tal que $W \times W \subset W^{\prime}$ e tal que $W \times W \subset F\left(\mathcal{U}^{\prime}\right)$ pois $\mathrm{F}$ deve ser bijetora.

\footnotetext{
${ }^{1}$ chamado de vizinhança totalmente normal em Carmo [Capítulo 3 - Teorema 3.7]
} 
Afirmamos que $W$ e $\epsilon^{\prime}$ assim obtidos satisfazem o enunciado do Teorema. De fato, se $q \in W$ e $\mathcal{B}\left(0, \epsilon^{\prime}\right) \subset T_{q} M$ então, como $F$ é um difeomorfismo de $\{q\} \times \mathcal{B}\left(0, \epsilon^{\prime}\right)$ sobre $\{q\} \times W,\{q\} \times W \subset F\left(\{q\} \times \mathcal{B}\left(0, \epsilon^{\prime}\right)\right)$.

Logo, pela definição de $F, F\left(\{q\} \times \mathcal{B}\left(0, \epsilon^{\prime}\right)\right)=\{q\} \times \exp _{q} \mathcal{B}\left(0, \epsilon^{\prime}\right)$ e portanto $W \subset \exp _{q} \mathcal{B}\left(0, \epsilon^{\prime}\right)$.

Conclui-se que:

(1) Todo ponto de $W$ pode ser ligado a $p$ por uma única geodésica;

(2) $W$ é uma vizinhança normal de cada um dos seus pontos ou seja, quaisquer dois pontos $q_{1}, q_{2} \in W$ podem ser ligados por uma única geodésica $\gamma:[0,1] \rightarrow M$ de comprimento menor que $\epsilon^{\prime}$ e que depende diferencialmente destes dois pontos no seguinte sentido:

dado $\left(q_{1}, q_{2}\right)$ existe um único $v \in T_{q_{1}} M$ (dado por $F^{-1}\left(q_{1}, q_{2}\right)=\left(q_{1}, v\right)$ com $\left.\exp _{q_{1}} v=q_{2}\right)$ que depende diferencialmente de $\left(q_{1}, q_{2}\right)$ e é tal que $\dot{\gamma}(0)=v$.

Em toda vizinhança normal de centro em $p$ existe um tipo especial de sistema de coordenadas que é particularmente simples em $M$ : se tomarmos um referencial ortonormal na origem de $T_{p} M$, a aplicação exponencial o "leva" através das geodésicas radiais para $M$, de acordo com o seguinte resultado. 
COROLÁRIO 1.34. Seja $\phi=\left(y_{1}, \cdots, y_{m}\right)$ um sistema de coordenadas cartesianas em $T_{p} M$ com relação à base canônica $\left\{e_{1}, \cdots, e_{m}\right\}$ ou seja

$$
\begin{aligned}
\phi: T_{p} M & \rightarrow \mathbb{R}^{m} \\
v & \rightarrow \phi(v)=\left(y_{1}(v), \cdots, y_{m}(v)\right)
\end{aligned}
$$

um isomorfismo. Então

$$
\begin{aligned}
\varphi: W \subset M & \rightarrow \mathbb{R}^{m} \\
q & \rightarrow \varphi(q)=\left(x_{1}(q), \cdots, x_{m}(q)\right)
\end{aligned}
$$

definida por $x_{i}=y_{i} \circ \exp _{p}^{-1}$, é um sistema de coordenadas local em $W$ chamado de sistema de coordenadas normal em torno de $p$.

Se $\gamma(t)=\exp _{p}(t v)$ é uma geodésica passando por $p$ em $t=0$ e tal que $v=\dot{\gamma}(0)$ então $\gamma(t)$ em coordenadas normais é dado por: $x_{i}(t)=t y_{i} i=1, \cdots, m$.

Observe que as geodésicas em $W$ que partem de $p$ são dadas por equações lineares pois $\varphi$ é uma isometria local. (Definição 1.5).

Assim, se $\varphi=\left(x_{1}, \cdots, x_{m}\right)$ é um sistema de coordenadas normal em $p \in M \mathrm{e}$ $\Gamma_{i j}^{k}$ são as componentes da conexão Levi-Civita de $g$ com relação a estas coordenadas então para todo $i, j, k=1, \cdots, m$

$$
\Gamma_{i j}^{k}(p)=0 \text { e } g_{i j}=\delta_{i j} .
$$

Decorre daí o seguinte resultado bem conhecido:

Sejam $p \in M, W$ uma vizinhança normal de $p$ e $B=\mathcal{B}(p, r) \subset W$.

Seja $\gamma:[0,1] \rightarrow B$ um segmento de geodésica ligando $p$ a $q$. Para qualquer outra curva $\alpha:[0,1] \rightarrow M$ ligando $p$ a q, tem-se que $l(\gamma) \leq l(\alpha)$ e se a igualdade vale então $\gamma([0,1])=\alpha([0,1])$.

Além disso, segmentos de curva que minimizam o comprimento de arco entre seus extremos são, a menos de parametrização, geodésicas.

([3, Capítulo 3 - Proposição 3.6, Corolário 3.9])

Diz-se que o segmento de geodésica $\gamma:[a, b] \rightarrow M$ é minimizante quando $l(\gamma) \leq l(\alpha)$ para toda curva $\alpha: I \rightarrow M$ diferenciável por partes ligando $\gamma(a)$ a $\gamma(b)$.

A função distância Riemanniana dist : $M \times M \rightarrow[0, \infty[$ é definida como o ínfimo dos comprimentos de todas as curvas diferenciáveis por partes ligando $p$ a $q$ :

$$
\operatorname{dist}(p, q)=\inf \left\{\mathcal{L}_{a}^{b}(\alpha), \alpha:[a, b] \rightarrow M \mid \alpha(a)=p \text { e } \alpha(b)=q\right\} .
$$

Assim, todo ponto de $M$ possui uma vizinhança normal $W$ tal que dois pontos quaisquer de $W$ podem ser ligados por uma única geodésica minimizante, e logo, que realiza a distância entre eles. 
3.2. Variações da Energia. Uma pergunta pertinente que se faz é:

Dada uma geodésica $\gamma:[0,1] \rightarrow M$, quais os instantes $t$ para os quais $\gamma$ minimiza o comprimento de arco desde $\gamma(0)$ até $\gamma(t)$ ?

Consideraremos este problema dentro de um contexto mais amplo, estudando as minimizações locais, ou seja, fixando $t$ e verificando se a restrição de $\gamma$ ao intervalo $[0, t]$ é de comprimento mínimo entre os segmentos que ligam $\gamma(0)$ a $\gamma(t)$ e que estão "suficientemente" próximos de $\gamma$, como será precisado a seguir. Neste sentido, as geodésicas são caracterizadas como soluções de um problema variacional.

DEFINIÇÃo 1.35. Seja $\alpha:[a, b] \rightarrow M$ uma curva diferenciável em $M$. Uma variação de $\alpha$ a um parâmetro é uma aplicação contínua

$$
f:]-\epsilon, \epsilon[\times[a, b] \rightarrow M
$$

tal que: $f(0, t)=\alpha(t), t \in[a, b]$.

Para cada $t \in[a, b]$, a curva $\left.f_{t}:\right]-\epsilon, \epsilon\left[\rightarrow M\right.$ dada por $f_{t}(s)=f(s, t)$ é chamada de curva transversal da variação $f$. O vetor velocidade de uma curva transversal em $s=0$ isto é,

$$
\left.\frac{\partial}{\partial s} f(s, t)\right|_{s=0}=V(t)
$$

é um campo vetorial diferenciável ao longo de $\alpha(t)$, dito o campo variacional de $f$.

Dado um campo $V \in \mathfrak{X}(M)$ pode-se sempre construir uma variação de $\alpha$ que o tenha como campo variacional, como será mostrado a seguir.

Proposição 1.36. Dado um campo $V \in \mathfrak{X}(M)$ ao longo de uma curva diferenciável $\alpha:[a, b] \rightarrow M$ existe uma variação $f:]-\epsilon, \epsilon[\times[a, b] \rightarrow M$ de $\alpha$ tal que $V(t)$ é o campo variacional de $f$.

Além disso, se $V(a)=V(b)=0$ é possivel escolher $f$ com extremos fixos $p$ e q. Tal variação é dita própria.

Prova. Como $\alpha([a, b]) \subset M$ é compacto, é possível determinar $\delta>0$ tal que $\exp _{\alpha(t)}, t \in[a, b]$, está bem definida para todo $v \in T_{\alpha(t)} M \operatorname{com}\|v\|<\delta$.

De fato, para cada $\alpha(t)$, pelo Teorema 1.33, existe uma vizinhança normal $W_{t}$ de $\alpha(t)$ e um número $\delta_{t}>0$ associado a esta vizinhança. A união $\bigcup_{t} W_{t}$ cobre o compacto $\alpha([a, b])$ e portanto, um número finito de $W_{t}^{\prime} s$, digamos $W_{1}, \cdots, W_{n}$, ainda cobre $\alpha([a, b])$. Basta escolher $\delta=\min \left\{\delta_{i}\right\}_{i=1, \cdots, n}$ onde $\delta_{i}>0$ é o número correspondente à vizinhança $W_{i}$.

Seja $N=\max _{t \in[a, b]}\|V(t)\|$ e $\epsilon<\frac{\delta}{N}$.

A chamada variação canônica própria de $\alpha$ é definida por

$$
\left.f(s, t)=\exp _{\alpha(t)} s V(t), s \in\right]-\epsilon, \epsilon[\mathrm{e} t \in[a, b] .
$$


Como $\|s V(t)\| \leq|s|\|V(t)\|<\epsilon N<\delta$, a aplicação $f:]-\epsilon, \epsilon[\times[a, b] \rightarrow M$ está bem definida. Além disso, $f(0, t)=\exp _{\alpha(t)} 0=\alpha(t)$ e o campo variacional de $f$ é dado por:

$$
\left.\frac{\partial}{\partial s} f(s, t)\right|_{s=0}=\left.\frac{\partial}{\partial s}\left(\exp _{\alpha(t)} s V(t)\right)\right|_{s=0}=\left(\mathrm{d} \exp _{\alpha(t)}\right)_{0} V(t)=V(t)
$$

e se $V(a)=V(b)=0$, a variação é própria, ou seja

$$
\left\{\begin{array}{c}
f(s, a)=\exp _{p} s V(a)=p \\
f(s, b)=\exp _{q} s V(b)=q
\end{array} \text { para todo } s \in\right]-\epsilon, \epsilon[.
$$

LEMA 1.37. Lema da Simetria.

Considere $f: A \subset \mathbb{R}^{2} \rightarrow M$ uma aplicação diferenciável isto é, $f(s, t)$ uma superfície parametrizada em $(M, g)$. Seja $\left(s_{0}, t_{0}\right)$ fixado em $A$ e denote

$$
\begin{aligned}
f_{s_{0}}^{\prime}(t) & =\left.\frac{\partial}{\partial t} f(s, t)\right|_{s=s_{0}} \text { por } \frac{\partial f}{\partial t} \\
f_{t_{0}}^{\prime}(s) & =\left.\frac{\partial}{\partial s} f(s, t)\right|_{t=t_{0}} \text { por } \frac{\partial f}{\partial s} .
\end{aligned}
$$

Então

$$
\frac{D}{d s} \frac{\partial f}{\partial t}=\frac{D}{d t} \frac{\partial f}{\partial s}
$$

Prova. Seja $(U, \varphi)$ um sistema de coordenadas em $f\left(s_{0}, t_{0}\right)$.

Se $\varphi \circ f(s, t)=\left(x_{1}(s, t), \cdots, x_{m}(s, t)\right)$, escreve-se

$$
\frac{\partial f}{\partial t}=\sum_{i=1}^{m} \frac{\partial x_{i}}{\partial t} \frac{\partial}{\partial x_{i}}, \quad \frac{\partial f}{\partial s}=\sum_{j=1}^{m} \frac{\partial x_{j}}{\partial s} \frac{\partial}{\partial x_{j}}
$$

obtendo-se:

$$
\begin{array}{r}
\frac{D}{d s} \frac{\partial f}{\partial t}=\frac{D}{d s}\left(\sum_{i=1}^{m} \frac{\partial x_{i}}{\partial t} \frac{\partial}{\partial x_{i}}\right)=\sum_{i=1}^{m} \frac{\partial^{2} x_{i}}{\partial s} \frac{\partial}{\partial x_{i}}+\sum_{i=1}^{m} \frac{\partial x_{i}}{\partial t} \nabla_{f_{t_{0}}^{\prime}(s)}^{\frac{\partial}{\partial x_{i}}} \\
=\sum_{i=1}^{m} \frac{\partial^{2} x_{i}}{\partial s} \frac{\partial}{\partial x_{i}}+\sum_{i, j=1}^{m} \frac{\partial x_{i}}{\partial t} \frac{\partial x_{j}}{\partial s} \nabla_{\frac{\partial}{\partial x_{j}}}^{\frac{\partial}{\partial x_{i}}}
\end{array}
$$

Pela simetria da conexão e pelo Teorema de Schwarz, vale a igualdade:

$$
\frac{D}{d s} \frac{\partial f}{\partial t}=\sum_{j=1}^{m} \frac{\partial^{2} x_{j}}{\partial t \partial s} \frac{\partial}{\partial x_{j}}+\sum_{i, j=1}^{m} \frac{\partial x_{j}}{\partial s} \frac{\partial x_{i}}{\partial t} \nabla_{\frac{\partial}{\partial x_{i}}}^{\frac{\partial}{\partial x_{j}}}=\frac{D}{d t} \frac{\partial f}{\partial s} .
$$

O Lema Fundamental do Cálculo de Variações estabelece que:

Seja $f:[0, T] \rightarrow \mathbb{R}$ uma função integrável. Uma condição necessária para que

$$
\int_{0}^{T} f(t) g(t) d t=0
$$

para toda função $g:[0, T] \rightarrow \mathbb{R}$ de classe $C^{1}$ com $g(0)=g(T)=0$ é que $f(t)=0$ para todo $t \in[0, T]$.

([18, Lema 2.4]) 
Corolário 1.38. Sejam $(M, g)$ uma variedade Riemanniana, $\alpha:[0, T] \rightarrow M$ uma curva diferenciável, e $W$ e $Z$ dois campos vetoriais atuando em $M$ ao longo de $\alpha$ isto é, $W(t), Z(t) \in T_{\alpha(t)} M$. Se

$$
\int_{0}^{T} g(W(t), Z(t)) d t=0
$$

para todo $\left.W\right|_{\alpha}$ então $Z(t)=0$ para quase todo $t \in[0, T]$.

A seguir é estabelecido o espaço das curvas ligando dois pontos fixados em $M$.

Uma aplicação $\alpha:[0,1] \rightarrow M$ é absolutamente contínua se, em coordenadas locais $(U, \varphi=x)$ tal que $\varphi(\alpha(0))=x(0)=0$, satisfaz a seguinte condição:

para todo $\epsilon>0$, existe $\delta>0$ tal que, dada qualquer sequência (crescente) finita $\left\{a_{i}\right\}_{0 \leq i \leq N}$ de pontos de $[0,1]$, ocorre que:

$$
\sum_{i=1}^{N}\left(a_{i}-a_{i-1}\right)<\delta \Longrightarrow \sum_{i=1}^{N}\left\|x\left(a_{i}\right)-x\left(a_{i-1}\right)\right\|<\epsilon .
$$

Daí, $\alpha$ admite derivada em quase todo lugar e além disso, para tal função, vale o Teorema Fundamental do Cálculo: $x(t)=\int_{0}^{t} \dot{x}(r) d r$ para todo $t$. Denota-se por $\Omega_{p, q}^{1}$ o espaço de todas as curvas absolutamente contínuas $\alpha:[0,1] \rightarrow$ M ligando dois pontos fixos $p=\alpha(0)$ e $q=\alpha(1)$ em $M$ e tendo $\int_{0}^{1} g(\dot{\alpha}, \dot{\alpha}) d t<+\infty$.

O espaço tangente a $\Omega_{p, q}^{1}$ em $\alpha$ é o espaço vetorial $T_{\alpha} \Omega_{p, q}^{1}$ de campos diferenciáveis $V$ ao longo de $\alpha$ tal que $V(0)=V(1)=0$ ou seja:

$$
T_{\alpha} \Omega_{p, q}^{1}=\left\{V:[0,1] \rightarrow T M, V(t) \in T_{\alpha(t)} M \text { e } V(0)=V(1)=0\right\} .
$$

O espaço tangente a $\Omega_{p, q}^{1}$ é um espaço de Hilbert de dimensão infinita. Um espaço de Hilbert é um espaço de Banach ${ }^{2}$ em que a norma é definida a partir de um produto interno ou seja, de uma forma bilinear definida positiva.

$\Omega_{p, q}^{1}$ tem a estrutura de uma variedade de Hilbert ([15, seção 13]) que, pode-se dizer grosseiramente, é uma variedade Riemanniana de dimensão infinita.

Para comparar o comprimento de arco de $\alpha \in \Omega_{p, q}^{1}$ com o comprimento das curvas vizinhas $\left\{f_{s}\right\}_{s \in \epsilon]-\epsilon, \epsilon[}$ de uma variação $\left.f:\right]-\epsilon, \epsilon[\times[0,1]$ de $\alpha$, define-se o funcional comprimento de arco $L\left[f_{s}\right]$ que a cada curva $f_{s}$ associa $\mathcal{L}_{0}^{1}\left(f_{s}\right) \in \mathbb{R}$.

Ou equivalentemente, a função $L:]-\epsilon, \epsilon[\rightarrow \mathbb{R}$ dada por

$$
L(s)=L\left[f_{s}\right]=\int_{0}^{1}\left\|f_{s}^{\prime}(t)\right\| d t=\int_{0}^{1} g\left(\frac{\partial f}{\partial t}(s, t), \frac{\partial f}{\partial t}(s, t)\right)^{\frac{1}{2}} d t .
$$

\footnotetext{
${ }^{2}$ Um espaço de Banach é um espaço vetorial normado com função distância definida por $d(x, y)=$ $\|x-y\|$ e tal que é um espaço métrico completo, no sentido de que toda sequência de Cauchy é convergente.
} 
DEFINIÇÃo 1.39. Seja $\alpha \in \Omega_{p, q}^{1}$. O funcional Energia $E$ em $\Omega_{p, q}^{1}$ é definido por

$$
E[\alpha]=\int_{0}^{1} \frac{1}{2}\|\dot{\alpha}(t)\|^{2} d t=\int_{0}^{1} \frac{1}{2} g(\dot{\alpha}(t), \dot{\alpha}(t)) d t .
$$

Esta é a chamada ação integral da curva $\alpha$ desde 0 até 1.

$\grave{A}$ ação integral $E[\alpha]$ falta o significado geométrico direto do funcional comprimento de arco $L[\alpha]$, mas seu uso simplifica os cálculos no seguinte sentido:

diferentemente de $L(s)$, a função $E(s)$ é sempre $C^{\infty}$. Além disso, não há prejuízo à análise da solução a ser determinada, pois $L(s)$ possui fórmulas análogas às da Energia para a primeira e segunda variações das curvas ([13, Capítulo 10]).

PROPOSIÇÃo 1.40. Primeira variação da Energia de uma curva.

Sejam $\alpha \in \Omega_{p, q}^{1}$ eV $\in T_{\alpha} \Omega_{p, q}^{1}$. A primeira variação do funcional Energia em $\alpha$ na direção de $V, \mathrm{~d} E[\alpha] V$, é dada por:

$$
\mathrm{d} E[\alpha] V=-\int_{0}^{1} g\left(V(t), \frac{D}{d t} \dot{\alpha}(t)\right) d t .
$$

Prova. Seja $f:]-\epsilon, \epsilon[\times[0,1] \rightarrow M$ uma variação própria de $\alpha$ com campo variacional $V \in T_{\alpha} \Omega_{p, q}^{1}$. Então $\mathrm{d} E[\alpha] V=\left.\frac{d}{d s}(E \circ f)(s, t)\right|_{s=0}$. Ou seja

$$
\begin{aligned}
E^{\prime}(0) & =\frac{d E}{d s}(0)=\left.\frac{d}{d s}(E \circ f)(s, t)\right|_{s=0} \\
& =\left.\frac{1}{2} \int_{0}^{1} \frac{d}{d s} g\left(\frac{\partial f}{\partial t}, \frac{\partial f}{\partial t}\right)\right|_{s=0} d t=\left.\int_{0}^{1} g\left(\frac{D}{d s} \frac{\partial f}{\partial t}, \frac{\partial f}{\partial t}\right)\right|_{s=0} d t
\end{aligned}
$$

Segue, pelo Lema da Simetria (1.37), que

$$
E^{\prime}(0)=\left.\int_{0}^{1} g\left(\frac{D}{d t} \frac{\partial f}{\partial s}, \frac{\partial f}{\partial t}\right)\right|_{s=0} d t=\int_{0}^{1} g\left(V^{\prime}(t), \dot{\alpha}(t)\right) d t .
$$

Pela compatibilidade da conexão com a métrica (Corolário 1.16):

$$
\begin{aligned}
\frac{d}{d t} g\left(\frac{\partial f}{\partial s}, \frac{\partial f}{\partial t}\right) & =g\left(\frac{D}{d t} \frac{\partial f}{\partial s}, \frac{\partial f}{\partial t}\right)+g\left(\frac{\partial f}{\partial s}, \frac{D}{d t} \frac{\partial f}{\partial t}\right) \text { tem-se que: } \\
E^{\prime}(0) & =\left.\int_{0}^{1} \frac{d}{d t} g\left(\frac{\partial f}{\partial s}, \frac{\partial f}{\partial t}\right)\right|_{s=0} d t-\left.\int_{0}^{1} g\left(\frac{\partial f}{\partial s}, \frac{D}{d t} \frac{\partial f}{\partial t}\right)\right|_{s=0} d t \\
& =\left.g(V(t), \dot{\alpha}(t))\right|_{0} ^{1}-\int_{0}^{1} g\left(V(t), \frac{D}{d t} \dot{\alpha}(t)\right) d t \\
& =-\int_{0}^{1} g\left(V(t), \frac{D}{d t} \dot{\alpha}(t)\right) d t
\end{aligned}
$$

pois $V(t)$ é o campo variacional da variação própria $f$ isto é, $V(0)=V(1)=0$.

DEFINIÇÃo 1.41. Uma curva $\alpha$ é crítica (ou elemento crítico) para o funcional Energia $E: \Omega_{p, q}^{1} \rightarrow \mathbb{R}$ se, para toda variação $f$ de $\alpha$,

$$
\left.\frac{d}{d s}(E \circ f)(s, t)\right|_{s=0}=E^{\prime}(0)=0 .
$$


Note que há uma analogia entre o que já sabíamos e a definição acima:

(1) $p \in M$ é um ponto crítico de uma função $F: M \rightarrow \mathbb{R}, C^{1}$, desde que $v[F]=\left.\frac{d}{d t}(F \circ \beta)(t)\right|_{t=0}=0$ para toda curva $\left.\beta:\right]-\epsilon, \epsilon[\rightarrow M$ passando por $p=\beta(0)$ com velocidade $v \in T_{p} M$ (Definição 1.24);

(2) $\alpha \in \Omega_{p, q}^{1}$ é um elemento crítico de $E: \Omega_{p, q}^{1} \rightarrow \mathbb{R}$ desde que $V[E]=0$ para toda variação $f:]-\epsilon, \epsilon\left[\times[0,1] \rightarrow M \operatorname{com} f_{0}(t)=\alpha(t)\right.$ e com campo variacional $\left.\frac{d}{d s} f(s, t)\right|_{s=0}=V(t) \in T_{\alpha(t)} M$. E portanto, $V[E]$ deve ser igual a $\left.\frac{d}{d s}(E \circ f)(s, t)\right|_{s=0}$.

O resultado dado a seguir é a confirmação de que as geodésicas em $\Omega_{p, q}^{1}$ são exatamente os pontos críticos do funcional Energia e, por decorrência, do funcional comprimento de $\operatorname{arco} L$.

COROLÁRIO 1.42. Uma curva $\alpha \in \Omega_{p, q}^{1}$ é crítica para E se e somente se $\alpha$ é uma geodésica. Ou de outro modo:

Uma curva de classe $C^{1} \alpha:[0,1] \rightarrow M$ é uma geodésica se e somente se, para toda variação própria $f$ de $\alpha$, tem-se que $E^{\prime}(0)=0$.

PROVA. Uma implicação é imediata: se $\alpha$ é uma geodésica, $\frac{D}{d t} \dot{\alpha}(t) \equiv 0$ e então $E^{\prime}(0)=-\int_{0}^{1} g\left(V(t), \frac{D}{d t} \dot{\alpha}(t)\right) d t=0$ para todo $V$ e, logo, para toda variação $f$ de $\alpha$. Portanto, por definição, $\alpha$ é um elemento crítico de $E$.

Suponha $E^{\prime}(0)=0$ para toda variação própria de $\alpha$. Para todo $V \in T_{\alpha} \Omega_{p, q}^{1}$, como $V(0)$ e $V(1)$ são nulos, $\exp _{\alpha(t)} s V(t)$ produz uma variação de $\alpha$ com extremos fixos e cujo campo variacional é $V$ (Proposição 1.36). Logo, por hipótese, $E^{\prime}(0)=-\int_{0}^{1} g\left(V(t), \frac{D}{d t} \dot{\alpha}(t)\right) d t=0$ para todo $V \in T_{\alpha} \Omega_{p, q}^{1}$.

Então, pelo Corolário $1.38, \frac{D}{d t} \dot{\alpha}=0$ para quase todo $t \in[0,1]$. Como $[0,1]$ é denso em $\mathbb{R}$, pode-se considerar $\frac{D}{d t} \dot{\alpha} \equiv 0$. E portanto $\alpha$ é uma geodésica em $M$.

Para estudar o comportamento de uma função $F: M \rightarrow \mathbb{R}, C^{2}$, próxima a um ponto crítico $p_{0}$ de $F$, considera-se o hessiano de $F$ em $p_{0}$ definido em $T_{p_{0}} M$. Analogamente, interessa tomar o hessiano ou segunda variação do funcional Energia $E$ em $\gamma$, elemento crítico de $E$, denotado por $\mathrm{d}^{2} E[\gamma]$ e definido em $T_{\gamma} \Omega_{p, q}^{1}$.

PROPOSIÇ̃̃o 1.43. Segunda variação da Energia de uma geodésica. Seja $\gamma:[0,1] \rightarrow M$ uma geodésica em $\Omega_{p, q}^{1}$ ef $\left.:\right]-\epsilon, \epsilon[\times[0,1] \rightarrow M$ uma variação própria de $\gamma$ com campo variacional $V$. Então

$$
\mathrm{d}^{2} E[\gamma](V, V)=\int_{0}^{1} g\left(V^{\prime}, V^{\prime}\right)+g(R(\dot{\gamma}, V) \dot{\gamma}, V) d t .
$$


PROVA. A prova será feita determinando-se

$$
E^{\prime \prime}(0)=\left.\frac{d^{2}}{d s^{2}}(E \circ f)(s, t)\right|_{s=0}=\mathrm{d}^{2} E[\gamma](V, V) .
$$

Pela Proposição 1.40 a primeira variação da Energia em uma curva $f_{s}(t)$ é dada por:

$$
E^{\prime}(s)=-\int_{0}^{1} g\left(\frac{\partial f}{\partial s}(s, t), \frac{D}{d t} \frac{\partial f}{\partial t}(s, t)\right) d t .
$$

Logo, obtem-se que:

$$
E^{\prime \prime}(s)=-\int_{0}^{1} g\left(\frac{D}{d s} \frac{\partial f}{\partial s}(s, t), \frac{D}{d t} \frac{\partial f}{\partial t}(s, t)\right)+g\left(\frac{\partial f}{\partial s}(s, t), \frac{D}{d s} \frac{D}{d t} \frac{\partial f}{\partial t}(s, t)\right) d t .
$$

Em $s=0$, como $\frac{D}{d t} \dot{\gamma}(t) \equiv 0$, segue que:

$$
E^{\prime \prime}(s)=-\int_{0}^{1} g\left(\frac{\partial f}{\partial s}(s, t), \frac{D}{d s} \frac{D}{d t} \frac{\partial f}{\partial t}(s, t)\right) d t .
$$

Como pelo Lema da Simetria (1.37) $\frac{D}{d s} \frac{\partial f}{\partial t}=\frac{D}{d t} \frac{\partial f}{\partial s} \Longrightarrow\left[\frac{\partial f}{\partial s}, \frac{\partial f}{\partial t}\right]=0$, a expressão do tensor curvatura de $g$ (Definição 1.19) é dada por:

$$
R\left(\frac{\partial f}{\partial t}, \frac{\partial f}{\partial s}\right) \frac{\partial f}{\partial t}=\frac{D}{d t} \frac{D}{d s} \frac{\partial f}{\partial t}-\frac{D}{d s} \frac{D}{d t} \frac{\partial f}{\partial t}
$$

e logo

$$
\frac{D}{d s} \frac{D}{d t} \frac{\partial f}{\partial t}=\frac{D}{d t} \frac{D}{d t} \frac{\partial f}{\partial s}-R\left(\frac{\partial f}{\partial t}, \frac{\partial f}{\partial s}\right) \frac{\partial f}{\partial t} .
$$

Como $\frac{d}{d t} g\left(V, \frac{D V}{d t}\right)=g\left(V, \frac{D^{2} V}{d t^{2}}\right)+g\left(\frac{D V}{d t}, \frac{D V}{d t}\right)$ e a variação é própria, obtem-se

$$
E^{\prime \prime}(0)=\int_{0}^{1} g\left(V^{\prime}, V^{\prime}\right)+g(R(\dot{\gamma}, V) \dot{\gamma}, V) d t .
$$

$\mathrm{d}^{2} E[\gamma](V, V)$ é também usualmente denotado por $I_{\gamma}[V, V]$.

Observe que a existência de $I[V, V]$ está garantida pela Proposição 1.43 pois $E^{\prime \prime}(0)$ depende apenas do campo variacional $V$ e não da particular variação $f$ considerada. E também que, de fato, $I[V, W]=\int_{0}^{1} g\left(V^{\prime}, W^{\prime}\right)+g(R(\dot{\gamma}, V) \dot{\gamma}, W) d t$, obtido a partir da forma quadrática pela identidade de polarização (Seção 2), é uma forma bilinear simétrica pois $g$ o é e para o tensor curvatura vale a propriedade (3-d) dada na Definição 1.19.

DEFINIÇÃo 1.44. Índice Morse

Dada uma aplicação $F, C^{2}$, em uma variedade diferenciável X e um ponto crítico $x \in X$ de $F$, então o índice Morse de $F$ em $x$ é o índice da segunda variação $\mathrm{d}^{2} F(x)$ em $T_{x} X$, que é o supremo das dimensões dos subespaços de $T_{x} X$ em que a forma bilinear simétrica $\mathrm{d}^{2} F(x)$ é definida negativa. 
Assim, o índice Morse de E em $\gamma$ é o supremo das dimensões dos subespaços de $T_{\gamma} \Omega_{p, q}^{1}$ nos quais $\mathrm{d}^{2} E[\gamma]$ é definido negativo.

Se $\gamma:[0,1] \rightarrow M$ é uma geodésica minimizante (por exemplo em uma vizinhança normal de $\gamma(0))$ e $V \in T_{\gamma} \Omega_{p, q}^{1}$ então, para toda variação própria $f$ de $\gamma$ associada a $V$, o funcional $E$ tem um mínimo local para $\gamma$ e portanto, d ${ }^{2} E[\gamma]$ é semidefinido positivo e o índice de $E$ em $\gamma$ é igual a zero. Vale, assim, uma recíproca análoga à da Proposição 1.28: Se $\gamma$ é uma geodésica minimizante ligando $p$ a $q$ então $\mathrm{d}^{2} E[\gamma]$ é semidefinido positivo.

De fato, se $\gamma$ é ponto de mínimo do funcional Energia então

$$
E\left[f_{s}\right] \geq E[\gamma]=E\left[f_{0}\right] .
$$

Portanto, como d $E[\gamma]=0, \mathrm{~d}^{2} E[\gamma]=\left.\frac{d^{2}}{d s^{2}}(E \circ f)(s, t)\right|_{s=0} \geq 0$.

Até aqui apenas situamos nossa questão inicial como um problema variacional. A teoria introduzida a seguir permitirá respondê-la.

\subsection{Conjugação e Campos de Jacobi.}

DEFINIÇÃo 1.45. Um campo de Jacobi $J$ é um campo vetorial ao longo de uma geodésica $\gamma:[0,1] \rightarrow M$ que satisfaz a equação de Jacobi:

$$
\frac{D^{2} J}{d t^{2}}-R(\dot{\gamma}, J) \dot{\gamma}=0
$$

onde $\left.J\right|_{\gamma(t)}=J(t)$ e R é o tensor curvatura de g, dado na Definição 1.19:

$$
R(X, Y) Z=\nabla_{X} \nabla_{Y} Z-\nabla_{Y} \nabla_{X} Z-\nabla_{[X, Y]} Z \text {. }
$$

Observe que $J$ satisfaz uma equação diferencial que corresponde a um sistema linear de $2 m$ equações de primeira ordem, e portanto, existem $2 m$ campos de Jacobi linearmente independentes ao longo de $\gamma$, e $J(t)$ é unicamente determinado pelas condições iniciais $J(0)$ e $J^{\prime}(0)=\frac{D J}{d t}(0)$.

LEMA 1.46. Sejam $p \in M, v \in T_{p} M$ e $w \in T_{v}\left(T_{p} M\right) \simeq T_{p} M$. Se fé uma superfície parametrizada em $M$, definida por

$$
\begin{aligned}
f:]-\epsilon, \epsilon[\times[0,1] & \rightarrow M \\
(s, t) & \rightarrow f(s, t)=\exp _{p} t(v+s w)
\end{aligned}
$$

então

$$
\left.\frac{\partial}{\partial s} f(s, t)\right|_{s=0}
$$

é um campo ao longo da geodésica $\gamma(t)=$ exp tv e satisfaz a equação de Jacobi. 
PRova. $\gamma:[0,1] \rightarrow M$ é a única geodésica passando por $p$ em $t=0$ e com $\dot{\gamma}(0)=v$. A família de geodésicas $\gamma_{s}(t)=\exp _{p} t(v+s w)$ é uma variação de $\gamma$ a um parâmetro tal que: $\gamma_{0} \equiv \gamma$ e satisfazendo:

$$
\begin{aligned}
& \left\{\begin{array}{l}
\gamma_{s}(0)=p \\
\gamma_{s}(1)=q(s)=\exp _{p}(v+s w)
\end{array} \quad \text { para todo } s \in\right]-\epsilon, \epsilon[\mathrm{e} \\
& \left.\left.\quad \frac{\partial}{\partial s} f(s, t)\right|_{s=0}=\left.\frac{\partial}{\partial s} \gamma_{t}(s)\right|_{s=0}=(\operatorname{dexp})_{p}\right)_{t v} t w=J(t), t \in[0,1]
\end{aligned}
$$

é o campo variacional de $f$ ao longo de $\gamma(t)$, segundo a Definição 1.35 .

Denotando $\dot{\gamma}_{s_{0}}(t)=\frac{\partial}{\partial t} f\left(s_{0}, t\right)$ para $s_{0}$ fixado em $]-\epsilon, \epsilon\left[\right.$ por $\frac{\partial f}{\partial t} \mathrm{e}$

$$
\dot{\gamma}_{t_{0}}(s)=\frac{\partial}{\partial s} f\left(s, t_{0}\right) \text { para } t_{0} \text { fixado em }[0,1] \text { por } \frac{\partial f}{\partial s}
$$

pelo Lema da Simetria (1.37) $\left[\frac{\partial f}{\partial s}, \frac{\partial f}{\partial t}\right]=0$, e o tensor curvatura de $g$ é dado por:

$$
R\left(\frac{\partial f}{\partial t}, \frac{\partial f}{\partial s}\right) \frac{\partial f}{\partial t}=\frac{D}{d t} \frac{D}{d s} \frac{\partial f}{\partial t}-\frac{D}{d s} \frac{D}{d t} \frac{\partial f}{\partial t} .
$$

Considerando que:

$$
\begin{aligned}
\frac{D}{d t} \dot{\gamma}_{s}(t) & =\frac{D}{d t} \frac{\partial}{\partial t} f(s, t) \equiv 0 \\
\frac{D}{d s} & \left(\frac{D}{d t} \frac{\partial f}{\partial t}\right)=\frac{D}{d t} \frac{D}{d t} \frac{\partial f}{\partial s}-R\left(\frac{\partial f}{\partial t}, \frac{\partial f}{\partial s}\right) \frac{\partial f}{\partial t}=0 .
\end{aligned}
$$

Então, em $s=0$, conclui-se que $J$ é um campo ao longo de $\gamma_{0}=\gamma$ que satisfaz

$$
\frac{D^{2} J}{d t^{2}}-R(\dot{\gamma}, J) \dot{\gamma}=0
$$

e portanto, por definição, é um campo de Jacobi.

Note que neste Lema:

(1) são dadas as seguintes condições iniciais para $J(t):\left\{\begin{array}{l}J(0)=0 \\ J^{\prime}(0)=\left.\frac{D J}{d t}\right|_{t=0}=w\end{array}\right.$ O campo de Jacobi $J$ ao longo de $\gamma$, que é o campo variacional de $f$, fica assim definido a partir do vetor $w \in T_{p} M$ dado e está unicamente determinado.

(2) $v(s)=v+s w$ é uma curva em $T_{p} M \operatorname{com} v(0)=v$ e $v^{\prime}(0)=w$.

Logo, $v=\dot{\gamma}(0)$ e $w=J^{\prime}(0)$ são ortogonais em $T_{p} M$. Na verdade, prova-se que:

$g\left(J^{\prime}(0), \dot{\gamma}(0)\right)=0 \Longleftrightarrow g(J, \dot{\gamma}) \equiv 0$. Este fato permite estudar a variação das geodésicas que partem de $p$ no sentido do seu afastamento transversal em relação a $\gamma$ isto é, a velocidade de afastamento das geodésicas que partem de $p$, dada por $\|J(t)\|$, relacionando-a com a curvatura seccional em $p$ segundo o plano $\sigma$ gerado por $\left\{J^{\prime}, \dot{\gamma}\right\}$, que é uma base ortonormal de cada $\sigma \subset T_{\gamma(t)} M$ e logo, ao longo de $\gamma$.

([3, Capítulo 5 - Corolário 2.9]) 
DEFINIÇÃo 1.47. Sejam $\gamma:[0,1] \rightarrow M$ uma geodésica em $M$ e $\left.\left.t_{0} \in\right] 0,1\right]$. $O$ ponto $\gamma\left(t_{0}\right)$ é dito conjugado a $\gamma(0)$ ao longo de $\gamma$ se existe um campo de Jacobi $J$, não nulo, ao longo de $\gamma$ tal que $J(0)=J\left(t_{0}\right)=0$.

O número máximo de campos de Jacobi linearmente independentes ao longo de $\gamma$, que se anulam em 0 e em $t_{0}$, é a multiplicidade do ponto conjugado $\gamma\left(t_{0}\right)$.

Observe que se $\gamma\left(t_{0}\right)$ é conjugado a $\gamma(0)$ então $\gamma(0)$ é conjugado a $\gamma\left(t_{0}\right)$, e assim:

Dois pontos $p$ e q são ditos conjugados em $M$ se existe uma geodésica $\gamma:[0,1] \rightarrow$ M ligando-os e tal que $p$ e q são conjugados ao longo de $\gamma$.

LEMA 1.48. O conjunto de campos de Jacobi $\left\{J_{i}(t): J_{i}(0)=0\right\}_{i=1, \cdots, k}$ é linearmente independente se e somente se $\left\{J_{1}^{\prime}(0), \cdots, J_{k}^{\prime}(0)\right\}$ é linearmente independente.

PRova. Seja $\left\{J_{1}, \cdots, J_{k}\right\}$ com $J_{i}(0)=0, i=1, \cdots, k$ l.i.

Os campos de Jacobi $J_{i}$ tal que $J_{i}(0)=0$, pelo Lema 1.46, são da forma

$$
J_{i}(t)=\left(\operatorname{dex} p_{p}\right)_{t v} t w_{i} \text { onde } w_{i}=J_{i}^{\prime}(0) \neq 0, i=1, \cdots, k .
$$

Suponha que $\left\{J_{i}^{\prime}(0)\right\}=\left\{w_{i}\right\}, i=1, \cdots, k$ é l.d. Então existe

$$
w_{l}=\sum_{\substack{i \neq l \\ i=1}}^{k} a_{i} w_{i}
$$

e como $\left(\mathrm{d} e x p_{p}\right)_{t v}$ é linear:

$$
J_{l}(t)=\left(\mathrm{d} \exp _{p}\right)_{t v} t w_{l}=\sum_{\substack{i=1 \\ i \neq l}}^{k} a_{i}\left(\mathrm{~d} \exp p_{p}\right)_{t v} t w_{i}=\sum_{\substack{i=1 \\ i \neq l}}^{k} a_{i} J_{i}(t)
$$

o que contradiz a hipótese de que $\left\{J_{i}(t)\right\}_{i=1, \cdots, k}$ é l.i.

A prova da recíproca é análoga.

Dados $J(0)=0$ e $J^{\prime}(0) \neq 0$, o campo de Jacobi $J$ que satisfaz a equação diferencial (1.7) fica unicamente determinado entre as $2 m$ soluções l.i. existentes.

Observe que $\dot{\gamma}(t)$ e $t \dot{\gamma}(t)$ são campos de Jacobi ao longo de $\gamma$. O primeiro nunca se anula, pois $\gamma$ é uma curva regular, e tem derivada zero. O segundo é nulo se e somente se $t=0$. Como são linearmente dependentes, no total de $2 m$ campos de Jacobi l.i. existentes, considere apenas $J(t)=t \dot{\gamma}(t)$.

Escolhidos $m J_{i}^{\prime}(0)$ 's l.i., pelo Lema 1.48, há $m$ escolhas de $J_{i}$ 's 1.i. com $J_{i}(0)=0$. Assim, existem exatamente $m$ campos de Jacobi l.i. ao longo de $\gamma$ que se anulam em $\gamma(0)$ e como $J(t)=t \dot{\gamma}(t)$ nunca se anula para $t \neq 0$, a multiplicidade de um ponto conjugado nunca excede $m-1$. 
DEFINIÇÃO 1.49. $q$ é um valor regular de $\exp _{p}: T_{p} M \rightarrow M s e$, para $\left.v_{0} \in \exp _{p}^{-1}(q),(\operatorname{dexp})_{p}\right)_{v_{0}}: T_{v_{0}}\left(T_{p} M\right) \simeq T_{p} M \rightarrow T_{\exp _{p} v_{0}} M \simeq T_{q} M \dot{e}$ sobrejetora sobre $T_{q} M$.

PROPOSIÇã̃o 1.50. Seja $\gamma:[0,1] \rightarrow M$ uma geodésica com $\gamma(0)=p e$ $\dot{\gamma}(0)=v$. O ponto $\left.\left.q=\gamma\left(t_{0}\right), t_{0} \in\right] 0,1\right]$, é conjugado a $p$ ao longo de $\gamma$ se $e$ somente se qé valor crítico de exp .

Além disto, a multiplicidade de q como ponto conjugado de pé igual à dimensão do núcleo da aplicação linear $\left(\operatorname{dexp} p_{p}\right)_{t_{0} v}$.

PROVA. Por definição, $q=\gamma\left(t_{0}\right)$ é conjugado a $p$ ao longo de $\gamma$ se e somente se existe um campo de Jacobi $J$, não nulo, ao longo de $\gamma \operatorname{com} J(0)=J\left(t_{0}\right)=0$.

Pelo Lema 1.46, J $(t)=\left(\operatorname{dexp}_{p}\right)_{t v} t w$ é um campo de Jacobi ao longo de $\gamma$ com $J(0)=0$ e $w=J^{\prime}(0) \neq 0$. Portanto $q=\gamma\left(t_{0}\right)$ é conjugado a $p$ se e somente se $J\left(t_{0}\right)=\mathrm{d}\left(\exp _{p}\right)_{t_{0} v} t_{0} w=0$, isto é, se e somente se $v_{0}=t_{0} v$ é ponto crítico de $\exp _{p}$. Ou seja, $\exp _{p} v_{0}=\gamma\left(t_{0}\right)=q$ é valor crítico de $\exp _{p}$.

Como $q$ é valor crítico de $e_{x} p_{p}$, dado $v_{0} \in \exp _{p}^{-1}(q),\left(\mathrm{d} \exp p_{p}\right)_{v_{0}}$ não é sobrejetora em $T_{p} M$ (Definição 1.49) e como $\left(\operatorname{dex} p_{p}\right)_{v_{0}}$ é linear e $\operatorname{dim} T_{p} M=\operatorname{dim} T_{q} M=m$, segue que $\left(\operatorname{dex} p_{p}\right)_{v_{0}}$ não é injetora, ou seja, seu núcleo não se reduz ao vetor nulo. Logo, existe $w_{0} \neq 0$ em $T_{p} M$ tal que $\left(\mathrm{d} e x p_{p}\right)_{v_{0}} w_{0}=0=J\left(t_{0}\right)$ pelo Lema 1.46. Então Nuc $\left(\left(\operatorname{dex} p_{p}\right)_{v_{0}}\right)=\left\{w_{i}=J_{i}^{\prime}(0): J_{i}\left(t_{0}\right)=0\right\}_{i=1, \cdots, k}$.

Pelo Lema $1.48 \operatorname{dim} \operatorname{Nuc}\left(\left(\operatorname{dex} p_{p}\right)_{v_{0}}\right)=k$ é igual ao número de campos de Jacobi $J_{i}(t)$, com $J_{i}(0)=0, i=1, \cdots, k$ l.i. e satisfazendo também $J_{i}\left(t_{0}\right)=0$. Este número é, por definição, a multiplicidade de $q=\gamma\left(t_{0}\right)$.

Seja $\gamma$ um ponto crítico para o funcional Energia em $\Omega_{p, q}^{1}$ (Corolário 1.42).

O espaço nulo de $\mathrm{d}^{2} E[\gamma]$, denotada por $I$, é o subespaço de $T_{\gamma} \Omega_{p, q}^{1}$ dado por: Nuc $(I)=\left\{V \in T_{\gamma} \Omega_{p, q}^{1}: I[V, W]=0\right.$ para todo $\left.W \in T_{\gamma} \Omega_{p, q}^{1}\right\}$ (Definição 1.25).

A dimensão de Nuc $(I)$ é dita a nulidade de $I$. Assim, a nulidade de $I$ é positiva se e somente se $I$ é uma forma bilinear degenerada. Portanto $\gamma$ é um ponto crítico não-degenerado de $E$ se e somente se $\operatorname{Nuc}(I)=\{0\}$.

Proposição 1.51. O espaço nulo de I é constituído por Campos de Jacobi. Ou seja, $J \in N u c(I)$ se e somente se J é um campo de Jacobi.

Prova. Se $J \in \operatorname{Nuc}(I)$ então $I[J, W]=0$ para todo $W \in T_{\gamma} \Omega_{p, q}([0, T], M)$. Por um argumento padrão de análise (boot-strap) não é difícil mostrar que $J$ é de classe $C^{2}$ e portanto

$$
\int_{0}^{1} g\left(J^{\prime}, W^{\prime}\right) d t=-\int_{0}^{1} g\left(J^{\prime \prime}, W\right) d t .
$$


Segue, pela Proposição 1.43, que

$$
I[J, W]=\int_{0}^{1} g\left(-J^{\prime \prime}, W\right)+g(R(\dot{\gamma}, J) \dot{\gamma}, W) d t=0
$$

para todo $W \in T_{\gamma} \Omega_{p, q}([0, T], M)$. Pelo Corolário 1.38 , tem-se que

$$
\frac{D^{2}}{d t^{2}} J-R(\dot{\gamma}, J) \dot{\gamma}=0
$$

para quase todo $t \in[0, T]$. Então $J$ é um campo de Jacobi (Definição 1.45). Se $J$ é um campo de Jacobi é imediato que $J \in$ Nuc $(I)$.

COROLÁRIO 1.52. I é uma forma bilinear degenerada se e somente se $p$ e $q$ são conjugados ao longo de $\gamma$. A nulidade de I é igual à multiplicidade de $p$ e $q$ como pontos conjugados; e logo a nulidade é finita.

Enuncia-se a seguir o importante Teorema do Índice, que é uma generalização de um resultado clássico de Jacobi que afirma que um segmento de geodésica minimiza o comprimento de arco em relação às curvas "vizinhas" de mesmas extremidades se e só se um tal segmento não possui pontos conjugados.

TEOREMA 1.53. Teorema do Índice (Morse)

$O$ índice Morse de $E$ é igual ao número de pontos $\gamma(t), t \in] 0,1[$, que são conjugados a $\gamma(0)$ ao longo de $\gamma$; cada ponto conjugado contado com sua multiplicidade. Este indice é finito.

([12, Teorema 15.1])

COROLÁRIO 1.54. Um segmento de geodésica $\gamma:[0,1] \rightarrow M$ pode conter apenas um número finito de pontos que são conjugados a $\gamma(0)$ ao longo de $\gamma$. Ou, em outras palavras: $O$ conjunto dos pontos conjugados ao longo de uma geodésica é um conjunto discreto.

Quanto à nossa questão inicial: dada uma geodésica $\gamma:[0,1] \rightarrow M$, para quais instantes $\gamma$ minimiza o comprimento de arco desde $p=\gamma(0)$ até $q=\gamma(t)$ ? Para $t \in] 0,1]$ desde que $p$ e $q$ sejam não-conjugados ao longo de $\gamma$.

De fato, pois neste caso, pelo Corolário $1.52, \operatorname{Nuc}(I)=\{0\}$ isto é, $\gamma$ é um ponto crítico não-degenerado de $E$. Pelo Teorema do Índice, o índice de $E$ em ]0, 1] é zero e segue que $\mathrm{d}^{2} E[\gamma]$ é definido positivo (Definição 1.44). Portanto $\gamma$ é um ponto de mínimo de $E$ em $\Omega_{p, q}^{1}$ ou seja, $\left.\gamma\right|_{[0, t]}$ é um segmento minimizante ligando $p$ a $q$.

\section{Completude}

PRoposição 1.55. A função dist $: M \times M \rightarrow \mathbb{R}^{+}$.dada por

$$
\operatorname{dist}(p, q)=\inf \left\{\mathcal{L}_{a}^{b}(\alpha), \alpha:[a, b] \rightarrow M \mid \alpha(a)=p \text { e } \alpha(b)=q\right\}
$$

é uma distância em $(M, g)$, isto é, satisfaz as propriedades de uma função distância: 
(1) $\operatorname{dist}(x, y)=\operatorname{dist}(y, x)$ para todo $x, y \in M$;

(2) $\operatorname{dist}(x, y) \geq 0$ e $\operatorname{dist}(x, y)=0 \Longleftrightarrow x=y$;

(3) $\operatorname{dist}(x, z) \leq \operatorname{dist}(x, y)+\operatorname{dist}(y, z)$ para todo $x, y, z \in M$.

Além disso, dist é contínua e a família das bolas métricas $\mathcal{B}(p, \epsilon)=\{q \in M$ : $\operatorname{dist}(p, q)<\epsilon\}$ para todo $\epsilon>0$ e $p \in M$ forma uma base para a topologia inicial da variedade. Portanto a topologia do espaço métrico ( $M$, dist) coincide com a topologia inicial de $M$.

([3, Capitulo 7 - Proposição 2.6])

TEOREMA 1.56. Teorema de Hopf-Rinow.

Seja $(M, g)$ uma variedade Riemanniana, conexa, com distância dist. Dado $p \in M$, as seguintes afirmações são equivalentes:

(1) $\exp _{p}$ está definida em todo $T_{p} M$;

(2) Os subconjuntos fechados e limitados de ( $M$, dist) são compactos;

(3) ( $M$, dist) é um espaço métrico completo;

(4) $(M, g)$ é geodesicamente completa ${ }^{3}$ : toda geodésica está definida para todo valor do parâmetro $t \in \mathbb{R}$;

(5) Existe uma sucessão de compactos $\left\{K_{n}\right\}_{n \in \mathbb{N}}, K_{n} \subset K_{n+1}, \bigcup_{n \in \mathbb{N}} K_{n}=M$, e uma sequência $\left\{p_{n}\right\}_{n \in \mathbb{N}}$, tal que se $p_{n} \notin K_{n}$ então $\lim _{n \rightarrow \infty} \operatorname{dist}\left(p, p_{n}\right)=+\infty$.

Cada uma das afirmações acima implica que:

(6) $(M, g)$ é geodesicamente conexa e além disso, para todo $q \in M$, existe pelo menos uma geodésica minimizante $\gamma$ ligando $p$ a $q$ tal que $\operatorname{dist}(p, q)=l(\gamma)$.

([3, Capítulo 7 - Teorema 2.8])

Uma variedade Riemanniana é dita completa se ocorre qualquer das afirmações de (1) a (4).

Toda variedade diferenciável suporta uma estrutura Riemanniana completa. Este fato foi provado em 1961 por Nomizu e Ozeki: toda variedade Riemanniana é conformemente equivalente a uma variedade Riemanniana completa. ([19, Capítulo I])

DEFINIÇÃo 1.57. Um ponto $p_{0}$ de uma variedade Riemanniana, não-compacta e completa, é um polo se possui a propriedade de não ter pontos conjugados.

A existência de um polo $p_{0}$ em uma variedade, que também seja simplesmente conexa, garante vários resultados globais importantes, centralizados no Teorema de Hadamard-Cartan. Estes resultados tem interesse independente e serão apresentados em seguida.

TEOREMA 1.58. Teorema de Hadamard-Cartan

\footnotetext{
${ }^{3}$ as soluções maximais da equação $\nabla_{\dot{\gamma}} \dot{\gamma}$ estão definidas em toda reta real para todas as condições iniciais
} 
Seja $(M, g)$ uma variedade Riemanniana completa, simplesmente conexa, com curvatura seccional não-positiva em todo ponto. Então $M$ é difeomorfa a $\mathbb{R}^{m}$.

Além disso, existe uma línica geodésica ligando qualquer par de pontos de $M$.

Os dois lemas seguintes mostram, em conjunto, que a aplicação exponencial em todo ponto de uma variedade de curvatura não-positiva é um difeomorfismo local.

LEMA 1.59. Seja $(M, g)$ completa, com curvatura não-positiva. Então todo ponto de $M$ é um polo.

Prova. Sejam $\gamma:\left[0, t_{0}\right] \rightarrow M$ uma geodésica em $M$, ligando $p$ a $q$ e $J$ o único campo de Jacobi, não nulo, ao longo de $\gamma, \operatorname{com} J(0)=0$ e $J^{\prime}(0)=w \in T_{p} M$. Suponha que $q$ é conjugado a $p$. Então $J(0)=J\left(t_{0}\right)=0$.

Calcula-se:

$$
\frac{d^{2}}{d t^{2}} g(J, J)=2 \frac{d}{d t} g\left(\frac{D J}{d t}, J\right)=2 g\left(\frac{D^{2} J}{d t^{2}}, J\right)+2 g\left(\frac{D J}{d t}, \frac{D J}{d t}\right) ;
$$

pela Equação de Jacobi:

$$
\frac{d^{2}}{d t^{2}} g(J, J)=2\left\|J^{\prime}\right\|^{2}+2 g(R(\dot{\gamma}(t), J(t)) \dot{\gamma}(t), J(t))
$$

e pela definição de curvatura seccional (1.20), obtem-se que:

$$
\frac{d^{2}}{d t^{2}} g(J, J)=2\left\|J^{\prime}\right\|^{2}-2 K_{p}(\{\dot{\gamma}, J\})\|\dot{\gamma} \times J\|^{2}
$$

Como $K_{p}(\sigma) \leq 0$ para todo $\sigma \in T_{p} M$, segue que $\frac{d^{2}}{d t^{2}} g(J, J) \geq 0$ e logo, $\frac{d}{d t} g(J, J)$ é não-decrescente ao longo de $\gamma$.

Por hipótese, $J(0)=J\left(t_{0}\right)=0$ e daí, $\frac{d}{d t} g(J, J)(0)=\frac{d}{d t} g(J, J)\left(t_{0}\right)=0$.

Mas, para $0 \leq t \leq t_{0}, 0=\frac{d}{d t} g(J, J)\left(t_{0}\right) \geq \frac{d}{d t} g(J, J)(t) \geq \frac{d}{d t} g(J, J)(0)=0$,

e assim $\frac{d}{d t} g(J, J)(t) \equiv 0$, para $t \in\left[0, t_{0}\right]$.

Então $\|J\|^{2}=$ cte e como $\|J(0)\|=0$, segue que $\|J\| \equiv 0$, o que contradiz a existência de um campo de Jacobi não trivial. Portanto, o absurdo está em supor que $p$ tenha algum conjugado. Pela arbitrariedade de $p$, todo ponto de $M$ é um polo.

LEMA 1.60. Dada uma variedade completa $(M, g)$, se $p_{0} \in M$ é um polo então $\exp _{p_{0}}: T_{p_{0}} M \rightarrow M$ é um difeomorfismo local.

Prova. Como $M$ é completa, pelo Teorema de Hopf-Rinow (1.56),para todo $q \in M$ existe pelo menos uma geodésica minimizante $\gamma:[0,1] \rightarrow M$ ligando $p_{0}=\gamma(0)$ a $q=\gamma(1)$. Seja $\dot{\gamma}(0)=v_{0}$.

Dado um polo $p_{0}$ em $M$, por definição, $p_{0}$ e $q$ são não-conjugados para todo $q \in M$. Segue, pela Proposição 1.50, que $q$ é valor regular de $\exp _{p_{0}}: T_{p_{0}} M \rightarrow M$, isto é, pela Definição 1.49 , se $v \in \exp _{p_{0}}^{-1}(q)$ então $\left(\operatorname{dex} p_{p_{0}}\right)_{v}$ é sobrejetora sobre $T_{q} M$. Logo $\left(\operatorname{dex} p_{p_{0}}\right)_{v}$ é um isomorfismo, pois é linear e $\operatorname{dim} T_{p_{0}} M=\operatorname{dim} T_{q} M$. 
Pelo Teorema da Função Inversa, existem abertos $\mathcal{U}_{v_{0}} \subset T_{p_{0}} M$ e $U \subset M$ com $\exp _{p_{0}} v_{0}=q \in U$ tal que $\exp _{p_{0}}: \mathcal{U}_{v_{0}} \rightarrow U$ é um difeomorfismo.

O ponto crucial da demonstração do Teorema de Hadamard-Cartan é dado pelo seguinte resultado geral:

LEMA 1.61. Sejam $(M, g),\left(N, g^{\prime}\right)$ variedades Riemannianas conexas de mesma dimensão $m \geq 2$, sendo $M$ completa. Se $f: M \rightarrow N$ é uma isometria local, então $N$ é completa e fé uma aplicação de recobrimento. $\quad$ ([3, Capítulo 7 - Lema 3.3])

Este Lema (1.61) relaciona dois conceitos fundamentais:

(1) $f: \mathcal{U} \subset M \rightarrow N$ é uma isometria local, se $f$ é um difeomorfismo em $\mathcal{U}$ e logo, para todo $p \in \mathcal{U}, d f_{p}: T_{p} M \rightarrow T_{f(p)} N$ é um isomorfismo tal que

$g(v, w)_{p}=g^{\prime}\left(d f_{p}(v), d f_{p}(w)\right)_{f(p)}$ (Definição 1.5), e

(2) $f: M \rightarrow N$ é uma aplicação de recobrimento de $N$ se para todo $q \in N$ existe um aberto conexo $U \subset N, q \in U$ tal que $f$ é sobrejetora e

$$
f^{-1}(U)=\bigcup_{\alpha \in I} U_{\alpha}
$$

onde $U_{\alpha}$ é um aberto de $M$ e tal que $\left.f\right|_{U_{\alpha}}: U_{\alpha} \rightarrow U$ é um difeomorfismo local.

COROLÁRIO 1.62. Seja $(M, g)$ uma variedade Riemanniana completa, conexa, com dimensão $m \geq 2$. Suponha que existe $p_{0} \in M$ tal que $p_{0}$ é um polo de $M$. Então $\exp _{p_{0}}: T_{p_{0}} M \rightarrow M$ é uma aplicação de recobrimento (neste ponto).

Prova. Como $M$ é completa e conexa, pelo Teorema de Hopf-Rinow, $\exp _{p_{0}}: T_{p_{0}} M \rightarrow M$ está bem definida em todo $T_{p_{0}} M$ e é sobrejetora. Como $p_{0}$ é polo de $M$, pelo Lema 1.60, $\exp _{p_{0}}$ é um difeomorfismo local. Isto permite introduzir uma métrica Riemanniana em $T_{p_{0}} M$ tal que $f=\exp _{p_{0}}: T_{p_{0}} M \rightarrow M$ seja uma isometria local, tomando-se a chamada métrica "pull-back" de $g$ : para cada $u \in T_{p_{0}} M$, define-se $f^{*}(g)(u)(v, w)=g\left(\left(\operatorname{dex} p_{p_{0}}\right)_{u}(v),\left(\operatorname{dex} p_{p_{0}}\right)_{u}(w)\right)$.

E como tal métrica é completa, pois as geodésicas de $T_{p_{0}} M$ passando pela origem são retas, pelo Teorema de Hopf-Rinow $\left(T_{p_{0}} M, f^{*}(g)\right)$ é uma variedade Riemanniana completa. Portanto, pelo Lema 1.61, exp $p_{p_{0}}$ é uma aplicação de recobrimento.

Prova-se a seguir que $M$ é difeomorfa a $\mathbb{R}^{m}$, já que $T_{p_{0}} M$ e $\mathbb{R}^{m}$ são isomorfos:

PROPOSIÇão 1.63. Se $(M, g)$ é simplesmente conexa e completa e contém um polo $p_{0}$ então $\exp _{p_{0}}: T_{p_{0}} M \rightarrow M$ é um difeomorfismo global.

PROVA. Pelo Corolário 1.62, se $p_{0} \in M$ é um polo então $e x p_{p_{0}}$ é uma aplicação de recobrimento e como $M$ é simplesmente conexa e $T_{p_{0}} M$ é conexa, por uma propriedade de espaços de recobrimento, $\exp _{p_{0}}$ é um homeomorfismo global. Como, 
pelo Lema 1.60, $\exp _{p_{0}}$ também é um difeo local, conclui-se que $\exp _{p_{0}}: T_{p_{0}} M \rightarrow M$ é um difeomorfismo global.

Finalmente com o próximo lema, um resultado fundamental por si só, completase a prova do Teorema de Hadamard-Cartan.

LEMA 1.64. Dada $(M, g)$, simplesmente conexa e completa, se $p_{0} \in M$ é um polo então para todo $q \in M$ existe uma única geodésica $\gamma_{q}:[0,1] \rightarrow M$ ligando $p_{0}$ $a q$.

Prova. Pela Proposição 1.63, $\exp _{p_{0}}: T_{p_{0}} M \rightarrow M$ é um difeomorfismo e além disso, é uma isometria (Corolário 1.62). Como as isometrias preservam as geodésicas isto é, existe uma correspondência biunívoca entre as geodésicas (retas) de $\mathbb{R}^{m}$ passando pela origem e as de $M$ passando por $p_{0}$, conclui-se que, para todo $q \in M$, existe uma única geodésica $\gamma_{q}:[0,1] \rightarrow M$ ligando $p_{0}$ a $q$.

É importante lembrar que não são apenas variedades com curvatura não-positiva que possuem polos. O parabolóide $S: z=x^{2}+y^{2}$ (Exemplo 2.44) tem curvatura positiva e admite o polo $(0,0,0)$ e tal que as geodésicas que passam por $(0,0,0)$ são exatamente as intersecções de $S$ com os planos contendo o eixo $z$.

DEFINIÇÃO 1.65. Um raio em $(M, g)$, uma variedade Riemanniana completa e não-compacta, é uma geodésica $\gamma:\left[0, \infty\left[\rightarrow M\right.\right.$ tal que $\left.\gamma\right|_{[0, T]}$, normalizada, é minimizante para todo $T>0$ ou seja, dist $(\gamma(s), \gamma(t))=|s-t|$ para todo $s, t \geq 0$.

LEMA 1.66. Passando por todo ponto de uma variedade Riemanniana nãocompacta, completa, existe um raio.

Prova. Como $(M, g)$ é completa, é geodesicamente conexa ou seja, para todo par de pontos $p, q \in M$ existe $\gamma_{p q}$, normalizado e minimizante que os liga.

Considere uma sequência de pontos $\left\{p_{n}\right\}_{n \in \mathbb{N}}$ divergente ao infinito e os segmentos geodésicos $\gamma_{p p_{n}}$ correspondentes.

A sequência $\left\{\dot{\gamma}_{p p_{n}}(0)\right\}_{n \in \mathbb{N}}$ possui um ponto de acumulação $v \in T_{p} M$, pois, como $\left\|\dot{\gamma}_{p p_{n}}\right\|=1$, esta é uma sequência infinita contida num compacto de $T_{p} M$ e logo, pelo Teorema de Bolzano-Weierstrass, possui um ponto de acumulação. Além disso, $\|v\|=1$, pois $v=\lim _{n \rightarrow \infty} \dot{\gamma}_{p p_{n}}(0)$ e $\left\|\dot{\gamma}_{p p_{n}}\right\|=1$ para todo $n \in \mathbb{N}$.

Sejam $\gamma_{v}:[0,+\infty[\rightarrow M$ a geodésica partindo de p e tal que $\dot{\gamma}(0)=v$; e $\left\{\gamma_{p p_{n}}\right\}_{n \in \mathbb{N}}$ uma subsequência que convirja a $\gamma_{v}$. Pelo Teorema de Hopf-Rinow (1.56) existe uma sequência de compactos $\left\{K_{n}\right\}_{n \in \mathbb{N}}, K_{n} \subset K_{n+1}, \bigcup_{n \in \mathbb{N}} K_{n}=M$, e uma sequência $\left\{p_{n}\right\}_{n \in \mathbb{N}}$, tal que se $p_{n} \notin K_{n}$ então $\lim _{n \rightarrow \infty} \operatorname{dist}\left(p, p_{n}\right)=+\infty$. Mas como $p_{n} \in K_{i}$ para algum $i,\left\{\gamma_{p p_{n}}\right\}_{n \in \mathbb{N}}$ converge uniformemente a $\gamma_{v}$ em $K_{i}$ para 
todo $i \in \mathbb{N}$ fixado. Então $\gamma_{v}$ é um segmento geodésico normalizado e minimizante em todo $K_{i}, i \in \mathbb{N}$. Portanto $\gamma_{v}$ é um raio. Como $p$ é arbitrário, conclui-se que por todo ponto de $M$ passa um raio.

Recordemos o seguinte teorema da teoria das equações diferenciais:

TEOREMA 1.67. Dados um campo vetorial $X \in \mathfrak{X}(M)$, definido em um aberto $V$ de uma variedade $M$ e $p \in V$, existem:

(1) um aberto $V_{0} \subset V$ com $p \in V_{0}$;

(2) um número $\delta>0$;

(3) uma aplicação diferenciável $\psi:]-\delta, \delta\left[\times V_{0} \rightarrow V\right.$ tal que para cada $q \in V_{0}$, a curva que a cada $t$ associa $\psi(t, q)$ ou seja, $\left.\gamma_{q}:\right]-\delta, \delta[\rightarrow V$ definida por $\gamma_{q}(t)=\psi(t, q)$, é a única curva integral de $X$ que no instante $t=0$ passa por $q$, ou seja, $\dot{\gamma}_{q}(t)=X(\gamma(t))$ e $\dot{\gamma}_{q}(0)=X_{q}$ (Definição 1.I3).

A imagem $\gamma_{q}(]-\delta, \delta[)$ de uma curva integral de $X$ é chamada uma órbita ou trajetória de $X$. Diz-se também que $\gamma_{q}$ é uma solução do sistema dinâmico $\frac{d \gamma}{d t}=X(\gamma)$ com condição inicial $\gamma(0)=q$.

A aplicação obtida fixando $t, \psi_{t}: V_{0} \rightarrow V$ definida por $\psi_{t}(q)=\psi(t, q)$ é o fluxo (local) do campo $X$ em $V$.

DEFINIÇÃo 1.68. $O$ fluxo de um campo vetorial completo $X \in \mathfrak{X}(M)$ é a aplicação $\psi: \mathbb{R} \times M \rightarrow M$ dada por $\psi_{t}(q)=\psi(t, q)$, sendo $\gamma_{q}(t)=\psi(t, q)$ a curva integral maximal de $X$ que passa por $q$.

DEFINIÇÃo 1.69. Um campo vetorial $X \in \mathfrak{X}(M)$ é de Killing se e somente se as aplicações $\psi_{t}: M \rightarrow M$ definidas por $\psi_{t}(q)=\psi(t, q)$ forem isometrias locais, isto é, pela Definição 1.5, para cada $t \in \mathbb{R}$

$$
g\left(\mathrm{~d} \psi_{(t, p)}(v), \mathrm{d} \psi_{(t, p)}(w)\right)_{\psi(t, q)}=g(v, w)_{p}
$$

para todo $v, w \in T_{p} M$.

Prova-se que:

Se $X \in \mathfrak{X}(M)$ é um campo de Killing então $g\left(\nabla_{Y} X, Z\right)=-g\left(\nabla_{Z} X, Y\right)$ para todo $Y, Z \in \mathfrak{X}(M)$.

Além disso, $\left.X\right|_{\gamma(t)}$ é um campo de Jacobi ao longo da geodésica $\gamma$.

([19, Capítulo 5 - Teorema 6.1]). 


\section{CAPíTULO 2}

\section{Convexidade em Variedades Riemannianas}

A noção de convexidade é estabelecida por dois conceitos estreitamente relacionados: conjuntos convexos e funções convexas. Estes conceitos são naturalmente estendidos do plano ao $\mathbb{R}^{n}$ e a variedades em geral, o que será feito ao longo deste capítulo.

\section{Funções convexas em $\mathbb{R}$}

DEFINIÇÃo 2.1. Um subconjunto $A$ do $\mathbb{R}^{n}$ é dito convexo se para todo par de pontos $p, q \in A$, o segmento $\overline{p q}$ está contido em $A$.

DEFINIÇÃo 2.2. Uma função real $f: I \rightarrow \mathbb{R}$, definida no intervalo fechado $I$ é dita convexa se $f(\lambda x+(1-\lambda) y) \leq \lambda f(x)+(1-\lambda) f(y)$ para todo $\lambda \in[0,1] e$ para todo par $x, y \in I$.

Uma função $f: I \rightarrow \mathbb{R}$ é dita estritamente convexa se esta desigualdade for estrita para todo $\lambda \in] 0,1[$.

LEMA 2.3. $f: A \subset \mathbb{R}^{n} \rightarrow \mathbb{R}$ é convexa se e somente se

$S(f)=\{(x, y): f(x) \leq y\} \subset A \times \mathbb{R}$ é convexo.

PROVA. Suponha que $f$ é convexa.

Sejam $\left(x_{1}, y_{1}\right),\left(x_{2}, y_{2}\right) \in S(f)$. Logo $f\left(x_{1}\right) \leq y_{1}$ e $f\left(x_{2}\right) \leq y_{2}$.

Considere o segmento de reta ligando $x_{1}$ a $x_{2} \mathrm{em} A$ :

$r(t)=x_{1}+t\left(x_{2}-x_{1}\right)=(1-t) x_{1}+t x_{2}, t \in[0,1]$.

Como $f$ é convexa, $f(r(t)) \leq(1-t) f\left(x_{1}\right)+t f\left(x_{2}\right) \leq(1-t) y_{1}+t y_{2}$.

Segue que $\left(r(t),(1-t) y_{1}+t y_{2}\right) \in S(f)$ e portanto $S(f)$ é convexo.

Suponha agora que $S(f)$ é convexo. Sejam $x_{1}, x_{2} \in A$. Logo

$\left(x_{1}, f\left(x_{1}\right)\right),\left(x_{2}, f\left(x_{2}\right)\right) \in S(f)$. Além disso, $\left(r(t),(1-t) f\left(x_{1}\right)+t f\left(x_{2}\right)\right) \in S(f)$ o que implica que $f(r(t)) \leq(1-t) f\left(x_{1}\right)+t f\left(x_{2}\right)$. Portanto $f$ é convexa em $A$.

Serão provadas as seguintes propriedades básicas de funções convexas em $\mathbb{R}$ :

- Se $f: I \rightarrow \mathbb{R}$ é convexa em I então $f$ é contínua no interior de I.

- Se fé contínua então

$f$ é convexa $\Longleftrightarrow$ para todo $x, y \in I, f\left(\frac{1}{2} x+\frac{1}{2} y\right) \leq \frac{1}{2} f(x)+\frac{1}{2} f(y)$. 
- Se f é convexa em I então existem as derivadas à direita e à esquerda, $f_{+}^{\prime}$ e $f_{-}^{\prime}$, em todo ponto interior de $I$.

- Se $f$ é diferenciável, então $f$ é convexa $\Longleftrightarrow f^{\prime}$ é não-decrescente em $I$.

- Se $f$ admite derivada segunda em todos os pontos então $f$ é convexa $\Longleftrightarrow f^{\prime \prime}(x) \geq 0$, em todo $x \in I$.

Inicialmente mostra-se um resultado que será bastante útil nas demonstrações destas propriedades:

LEMA 2.4. Sejam $a, x, x_{0}, b \in I$, com $a<x<x_{0}<b$. Se $f: I \rightarrow \mathbb{R}$ é convexa então

$$
\frac{f(x)-f(a)}{x-a} \leq \frac{f\left(x_{0}\right)-f(a)}{x_{0}-a} \leq \frac{f\left(x_{0}\right)-f(x)}{x_{0}-x} \leq \frac{f(b)-f(x)}{b-x} \leq \frac{f(b)-f\left(x_{0}\right)}{b-x_{0}} .
$$

Prova. Como $f$ é convexa e $x=(1-\lambda) a+\lambda x_{0}$ para $\left.\lambda=\frac{x-a}{x_{0}-a}, \lambda \in\right] 0,1[$,

$$
\begin{aligned}
f(x) & \leq(1-\lambda) f(a)+\lambda f\left(x_{0}\right) \\
f(x)-f(a) & \leq \frac{x-a}{x_{0}-a}\left(f\left(x_{0}\right)-f(a)\right) \\
\frac{f(x)-f(a)}{x-a} & \leq \frac{f\left(x_{0}\right)-f(a)}{x_{0}-a} .
\end{aligned}
$$

Escrevendo $\lambda=\frac{x-a}{x_{0}-a}=\frac{\left(x-x_{0}\right)+\left(x_{0}-a\right)}{x_{0}-a}$

e como $1-\lambda=\frac{x_{0}-x}{x o-a}$, segue que:

$$
\begin{aligned}
& f(x) \leq(1-\lambda) f(a)+\lambda f\left(x_{0}\right) \\
& f(x) \leq \frac{x_{0}-x}{x_{0}-a} f(a)+\frac{\left[\left(x-x_{0}\right)+\left(x_{0}-a\right)\right]}{x_{0}-a} f\left(x_{0}\right) \\
& \left(f(x)-f\left(x_{0}\right)\right)\left(x_{0}-a\right) \leq\left(f(a)-f\left(x_{0}\right)\right)\left(x_{0}-x\right) \\
& \frac{f\left(x_{0}\right)-f(a)}{x_{0}-a} \leq \frac{f\left(x_{0}\right)-f(x)}{x_{0}-x} .
\end{aligned}
$$

Analogamente, tomando $x<x_{0}<b$, obtem-se que:

$$
\frac{f\left(x_{0}\right)-f(x)}{x_{0}-x} \leq \frac{f(b)-f(x)}{b-x} \leq \frac{f(b)-f\left(x_{0}\right)}{b-x_{0}} .
$$

PROPOSIÇão 2.5. Se $f: I \rightarrow \mathbb{R}$ é convexa então $f$ é contínua no interior de $I$.

PRovA. Seja $\left.x_{0} \in\right] a, b[\subset I$. Defina

$$
\begin{aligned}
& g:] a, b\left[\rightarrow \mathbb{R} \quad \text { por } \quad g(x)=\frac{f(x)-f(a)}{x-a}\left(x_{0}-x\right)\right. \\
& h:] a, b\left[\rightarrow \mathbb{R} \quad \text { por } \quad h(x)=\frac{f(b)-f(x)}{b-x}\left(x_{0}-x\right) .\right.
\end{aligned}
$$

Como $f$ é convexa em $I$, pelo Lema $2.4, g(x) \leq f\left(x_{0}\right)-f(x) \leq h(x)$ para todo $x \in] a, b\left[, x \neq x_{0}\right.$. E como $\lim _{x \rightarrow x_{0}} g(x)=\lim _{x \rightarrow x_{0}} h(x)=0$, segue que 
$\lim _{x \rightarrow x_{0}} f\left(x_{0}\right)-f(x)=0$. Portanto, $\lim _{x \rightarrow x_{0}} f(x)=f\left(x_{0}\right)$ e, pela arbitrariedade de $x_{0}, f$ é contínua no interior de $I$.

Proposição 2.6. Se $f: I \rightarrow \mathbb{R}$ é contínua então

$f$ é convexa $\Longleftrightarrow$ para todo $x, y \in I, f\left(\frac{1}{2} x+\frac{1}{2} y\right) \leq \frac{1}{2} f(x)+\frac{1}{2} f(y)$.

PROVA. Suponha que $f\left(\frac{1}{2} x+\frac{1}{2} y\right) \leq \frac{1}{2} f(x)+\frac{1}{2} f(y)$, para todo $x, y \in I$. Então, mostra-se por indução finita sobre $k=0,1, \ldots, 2^{m}-1, m \in \mathbb{N}$ que, para $\lambda_{k}=\frac{k}{2^{m}}$ :

$$
f\left(\lambda_{k} x+\left(1-\lambda_{k}\right) y\right) \leq \lambda_{k} f(x)+\left(1-\lambda_{k}\right) f(y) .
$$

Como $D=\left\{\frac{k}{2^{m}} \in \mathbb{Q}, 0 \leq k<2^{m}, m \in \mathbb{N}\right\}$ é denso em $[0,1]$, para cada $\lambda \in[0,1]$ existe uma sequência de números racionais $\lambda_{n} \in D$ tal que $\lim _{n \rightarrow \infty} \lambda_{n}=\lambda$.

Como $f$ é contínua,

$$
\begin{aligned}
f(\lambda x+(1-\lambda) y) & =\lim _{n \rightarrow \infty} f\left(\lambda_{n} x+\left(1-\lambda_{n}\right) y\right) \\
& \leq \lim _{n \rightarrow \infty}\left[\lambda_{n} f(x)+\left(1-\lambda_{n}\right) f(y)\right]=\lambda f(x)+(1-\lambda) f(y) .
\end{aligned}
$$

E portanto $f$ é convexa em I.

Se fé convexa em $I, f(\lambda x+(1-\lambda) y) \leq \lambda f(x)+(1-\lambda) f(y)$ para todo $x, y \in I$, e para todo $\lambda \in[0,1]$ e em particular para $\lambda=\frac{1}{2}$.

PRoposição 2.7. Se $f: I \rightarrow \mathbb{R}$ é convexa então existem $f_{+}^{\prime}$ e $f_{-}^{\prime}$ em todo ponto interior de $I$.

PROVA. Seja $\left.x_{0} \in\right] a, b[\subset I$. Considere a função $g:] x_{0}, b[\rightarrow \mathbb{R}$ dada por

$$
g(x)=\frac{f(x)-f\left(x_{0}\right)}{x-x_{0}} .
$$

A prova será feita mostrando-se que existe $\lim _{x \rightarrow x_{0+}} g(x)=f_{+}^{\prime}\left(x_{0}\right)$.

Como f é convexa, tomando $x_{0}<x_{1}<x_{2}<b$ no Lema 2.4, obtem-se que:

$$
\frac{f\left(x_{1}\right)-f\left(x_{0}\right)}{x_{1}-x_{0}} \leq \frac{f\left(x_{2}\right)-f\left(x_{0}\right)}{x_{2}-x_{0}}
$$

e logo $g$ é não-decrescente em $] x_{0}, b\left[\right.$. Para $a<x_{0}<x<b$ obtem-se também que:

$$
\frac{f\left(x_{0}\right)-f(a)}{x_{0}-a} \leq g(x) \leq \frac{f(b)-f\left(x_{0}\right)}{b-x_{0}}
$$

e logo $g$ é limitada em $] x_{0}, b[$.

Portanto, existe inf $\{g(x), x \in] x_{0}, b\left[\operatorname{com} x>x_{0}\right\}=\lim _{x \rightarrow x_{0+}} g(x)=f_{+}^{\prime}\left(x_{0}\right)$.

Analogamente, defina a função $h:] a, x_{0}[\rightarrow \mathbb{R}$ por

$$
h(x)=\frac{f\left(x_{0}\right)-f(x)}{x_{0}-x} .
$$

Como $h$ é monótona (não-crescente) e limitada em $] a, x_{0}[$ existe

$$
\lim _{x \rightarrow x_{0-}} h(x)=\sup \{h(x), x \in] a, x_{0}\left[, x<x_{0}\right\}=f_{-}^{\prime}\left(x_{0}\right) .
$$


LEMA 2.8. Se $f: I \rightarrow \mathbb{R}$ é uma função convexa em $I$ então $f_{+}^{\prime}$ e $f_{-}^{\prime}$ são não-decrescentes no interior de $I$.

Prova. Sejam $\left.x, y, t_{1}, t_{2}, t_{3} \in\right] a, b\left[\subset I\right.$ tal que $t_{1}<x<t_{2}<y<t_{3}$.

Pelo Lema 2.4, obtem-se as desigualdades abaixo, e como, pela Proposição 2.7, existem as derivadas laterais de $f$, segue que:

$$
\begin{aligned}
\frac{f(x)-f\left(t_{1}\right)}{x-t_{1}} & \leq \frac{f\left(t_{2}\right)-f(x)}{t_{2}-x} \\
& \leq \frac{f(y)-f(x)}{y-x} \leq \frac{f(y)-f\left(t_{2}\right)}{y-t_{2}} \leq \frac{f\left(t_{3}\right)-f(y)}{t_{3}-y} \\
f_{-}^{\prime}(x) & \leq f_{+}^{\prime}(x) \\
& \leq \frac{f(y)-f(x)}{y-x} \leq f_{-}^{\prime}(y) \leq f_{+}^{\prime}(y) .
\end{aligned}
$$

Portanto $f_{+}^{\prime}(x) \leq f_{+}^{\prime}(y)$ e $f_{-}^{\prime}(x) \leq f_{-}^{\prime}(y)$.

Proposição 2.9. Seja $f: I \rightarrow \mathbb{R}$ diferenciável. Então $f$ é convexa em I se e somente se $f^{\prime}$ é não-decrescente em $I$.

PROVA. Pelo Lema 2.8, $f_{+}^{\prime}(x) \leq f_{+}^{\prime}(y)$, para todo $\left.x, y \in\right] a, b[\subset I \operatorname{com} x<y$. Como $f$ é diferenciável $f^{\prime}(x)=f_{+}^{\prime}(x) \leq f_{+}^{\prime}(y)=f^{\prime}(y)$, e portanto, $f^{\prime}$ é nãodecrescente em $I$, pela arbitrariedade da escolha de $a$ e $b$.

Suponha agora que $f^{\prime}$ é não-decrescente em $] a, b[$.

Mostra-se a seguir que $\lambda f(x)+(1-\lambda) f(y)-f(\lambda x+(1-\lambda) y) \geq 0$, para $a<x<y<b, \lambda \in[0,1]$. E logo que $f$ é convexa em $[a, b]$.

De fato, pelo Teorema Fundamental do Cálculo, para cada $c \in] a, b[$, $f(x)-f(c)=\int_{c}^{x} f^{\prime}(t) d t$ para todo $\left.x \in\right] a, b\left[\right.$, e fazendo-se $g=f^{\prime}$, obtem-se que:

$$
\begin{aligned}
& \lambda f(x)+(1-\lambda) f(y)-f(\lambda x+(1-\lambda) y) \\
= & \lambda\left[f(c)+\int_{c}^{x} g(t) d t\right]+(1-\lambda)\left[f(c)+\int_{c}^{y} g(t) d t\right] \\
& -[\lambda+(1-\lambda)]\left[f(c)+\int_{c}^{\lambda x+(1-\lambda) y} g(t) d t\right] \\
= & -\lambda \int_{x}^{c} g(t) d t+(1-\lambda) \int_{c}^{y} g(t) d t \\
& -\lambda \int_{c}^{\lambda x+(1-\lambda) y} g(t) d t-(1-\lambda) \int_{c}^{\lambda x+(1-\lambda) y} g(t) d t \\
= & -\lambda \int_{x}^{\lambda x+(1-\lambda) y} g(t) d t+(1-\lambda) \int_{\lambda x+(1-\lambda) y}^{y} g(t) d t .
\end{aligned}
$$

Como $g$ é não-decrescente, vale para todo $\left.t_{0}, t_{1} \in\right] a, b[$ que

$$
g\left(t_{0}\right)\left(t_{1}-t_{0}\right) \leq \int_{t_{0}}^{t_{1}} g(t) d t \leq g\left(t_{1}\right)\left(t_{1}-t_{0}\right) .
$$


Segue que:

$$
\begin{aligned}
& \lambda f(x)+(1-\lambda) f(y)-f(\lambda x+(1-\lambda) y) \\
\geq & -\lambda g(\lambda x+(1-\lambda) y)[\lambda x+(1-\lambda) y-x] \\
& +(1-\lambda) g(\lambda x+(1-\lambda) y)[y-\lambda x+(1-\lambda) y] \\
= & g(\lambda x+(1-\lambda) y) \\
& {\left[-\lambda^{2} x-\lambda(1-\lambda) y+\lambda x+(1-\lambda) y-\lambda(1-\lambda) x+(1-\lambda)^{2} y\right]=0 . }
\end{aligned}
$$

Portanto, $f$ é convexa em $I$, pois $a$ e $b$ são arbitrários.

Como consequência da Proposição anterior (2.9) prova-se que o gráfico de uma função convexa $f$, diferenciável, situa-se acima de toda reta tangente, que é uma reta-suporte para $f$ em cada ponto que se considerar. E a partir deste resultado, demonstra-se a última propriedade enunciada. Assim, precisa-se estabelecer a noção de reta-suporte. Mas, para tanto, é interessante introduzir-se antes uma propriedade fundamental das funções convexas e que vai recomendar seu uso em estudos com abordagem global: a convexidade local é equivalente à convexidade global.

DEFINIÇão 2.10. Uma função $f: \mathbb{R} \rightarrow \mathbb{R}$ é dita localmente convexa se para todo $x \in \mathbb{R}$, existir um intervalo aberto $I_{x}$ que contenha $x$, no qual $f$ seja convexa.

LEMA 2.11. Sejam $I_{1}, I_{2}$ intervalos abertos de $I$, tal que $I_{1} \cap I_{2} \neq \emptyset$.

Considere $f: I_{1} \cup I_{2} \rightarrow \mathbb{R}$ com $f \mid I_{1}$ e $f \mid I_{2}$ convexas. Então $f$ é convexa em $I_{1} \cup I_{2}$.

Prova. Suponha que $f$ não seja convexa em $I_{1} \cup I_{2}$.

Então existem $\left.x_{0}, y_{0} \in I_{1} \cup I_{2}, \lambda \in\right] 0,1\left[\right.$ tal que para $z=(1-\lambda) x_{0}+\lambda y_{0}$ ocorre que $f(z)>(1-\lambda) f\left(x_{0}\right)+\lambda f\left(y_{0}\right)$.

Sem perda de generalidade, suponha que $x_{0} \in I_{1}, y_{0} \in I_{2} \backslash I_{1}$, pois $y_{0} \in I_{1}$ contradiz o fato de $f \mid I_{1}$ ser convexa; tome também $z \in I_{2}$ e $x_{0}<z<y_{0}$.

Como $f(z)>(1-\lambda) f\left(x_{0}\right)+\lambda f\left(y_{0}\right) \Longrightarrow f(z)-f\left(x_{0}\right)>\lambda\left(f\left(y_{0}\right)-f\left(x_{0}\right)\right)$ com $\lambda=\frac{z-x_{0}}{y_{0}-x_{0}}$, segue que:

$$
\frac{f\left(y_{0}\right)-f\left(x_{0}\right)}{y_{0}-x_{0}}<\frac{f(z)-f\left(x_{0}\right)}{z-x_{0}} .
$$

Escrevendo $\lambda=\frac{\left(z-y_{0}\right)+\left(y_{0}-x_{0}\right)}{y_{0}-x_{0}}$, como $1-\lambda=\frac{y_{0}-z}{y_{0}-x_{0}}$ e $z-y_{0}<0$, obtem-se que:

$$
\begin{aligned}
f(z) & >(1-\lambda) f\left(x_{0}\right)+\lambda f\left(y_{0}\right) \\
f(z) & >\frac{y_{0}-z}{y_{0}-x_{0}} f\left(x_{0}\right)+\left[\frac{\left(z-y_{0}\right)+\left(y_{0}-x_{0}\right)}{y_{0}-x_{0}}\right] f\left(y_{0}\right) \\
\left(y_{0}-x_{0}\right)\left(f(z)-f\left(y_{0}\right)\right) & >\left(z-y_{0}\right)\left(f\left(y_{0}\right)-f\left(x_{0}\right)\right) \\
\frac{f(z)-f\left(y_{0}\right)}{z-y_{0}} & <\frac{f\left(y_{0}\right)-f\left(x_{0}\right)}{y_{0}-x_{0}} .
\end{aligned}
$$


Portanto

$$
\frac{f(z)-f\left(y_{0}\right)}{z-y_{0}}<\frac{f\left(y_{0}\right)-f\left(x_{0}\right)}{y_{0}-x_{0}}<\frac{f(z)-f\left(x_{0}\right)}{z-x_{0}} .
$$

Como $I_{2}$ é aberto, existe $\delta>0$ tal que $x_{1}=z-\delta \in I_{2}$. Tome $\epsilon>0$ tal que $y_{1}=z+\epsilon<y_{0}$ satisfazendo, para $\delta$ suficientemente pequeno,

$$
\frac{f\left(y_{1}\right)-f\left(x_{1}\right)}{y_{1}-x_{1}}=\frac{f\left(y_{0}\right)-f\left(x_{0}\right)}{y_{0}-x_{0}} .
$$

Como $f \mid I_{2}$ é convexa e $x_{1}<z<y_{1}<y_{0}$, pelo Lema 2.4,

$$
\frac{f\left(y_{1}\right)-f\left(x_{1}\right)}{y_{1}-x_{1}} \leq \frac{f\left(y_{0}\right)-f(z)}{y_{0}-z} .
$$

Mas, então, pelas desigualdades acima e por construção, obtem-se que:

$$
\frac{f\left(y_{1}\right)-f\left(x_{1}\right)}{y_{1}-x_{1}} \leq \frac{f\left(y_{0}\right)-f(z)}{y_{0}-z}<\frac{f\left(y_{0}\right)-f\left(x_{0}\right)}{y_{0}-x_{0}}=\frac{f\left(y_{1}\right)-f\left(x_{1}\right)}{y_{1}-x_{1}} .
$$

E chega-se a um absurdo que foi devido a se supor inicialmente que $f$ não fosse convexa em $I_{1} \cup I_{2}$.

PRoposição 2.12. $f: \mathbb{R} \rightarrow \mathbb{R}$ é uma função convexa se e somente se $f$ é localmente convexa.

PRova. Como para cada $x \in \mathbb{R}$ existe uma vizinhança aberta $I_{x}$ tal que $\left.f\right|_{I_{x}}$ é convexa, segue que $f$ é localmente convexa.

Suponha $f$ localmente convexa e sejam $a, b \in \mathbb{R}$. Então, para todo $t_{i} \in[a, b], i \in$ $\mathbb{N}$, existe $\delta_{t_{i}}>0$ tal que, para $\left.K_{i}=\right] t_{i}-\delta_{t_{i}}, t_{i}+\delta_{t_{i}}\left[, f \mid K_{i}\right.$ é convexa.

Como a família de conjuntos abertos $\left\{K_{i}\right\}_{t_{i} \in[a, b]}$ é uma cobertura de $[a, b]$, um intervalo compacto, existe uma subcoleção finita $\left\{K_{i}\right\}_{i=1, \ldots, n}$ tal que $[a, b] \subset \bigcup_{i=1}^{n} K_{i}$.

Pelo Lema 2.11, $f$ é convexa em $K_{i} \cup K_{i+1}$ para todo $i=1, \ldots, n-1$.

Portanto $f$ é convexa em $[a, b]$. Como $a$ e $b$ são arbitrários, f é convexa em $\mathbb{R}$.

DEFINIÇÃo 2.13. Uma função afim $s: I \rightarrow \mathbb{R}$ é uma reta-suporte para $f: I \rightarrow \mathbb{R}$ em $x_{0} \in I$ se $s\left(x_{0}\right)=f\left(x_{0}\right)$ e $f(x) \geq s(x)$ para todo $x \neq x_{0}, x \in I$.

COROLÁRIO 2.14. $f: I \rightarrow \mathbb{R}$ é convexa se e somente se $f$ admite uma retasuporte em cada ponto de $I$.

Prova. Considere $f: J \rightarrow \mathbb{R}$ uma extensão de $f$ tal que $I$ esteja contido no interior de $J$. Como, pela Proposição $2.12 f$ é convexa em $J$, segue, pela demonstração do Lema 2.8 que, para $x_{0}, x \in$ interior de $J \operatorname{com} \mathrm{x}_{0}<\mathrm{x}$

$$
f_{-}^{\prime}\left(x_{0}\right) \leq f_{+}^{\prime}\left(x_{0}\right) \leq \frac{f(x)-f\left(x_{0}\right)}{x-x_{0}} .
$$


Assim, se $m \in\left[f_{-}^{\prime}\left(x_{0}\right), f_{+}^{\prime}\left(x_{0}\right)\right]$ então $\frac{f(x)-f\left(x_{0}\right)}{x-x_{0}} \geq m$.

Para $\mathrm{x}_{0}>\mathrm{x}$, ocorre que

$$
\frac{f\left(x_{0}\right)-f(x)}{x_{0}-x} \leq f_{-}^{\prime}\left(x_{0}\right) \leq f_{+}^{\prime}\left(x_{0}\right) .
$$

Assim, se $m \in\left[f_{-}^{\prime}\left(x_{0}\right), f_{+}^{\prime}\left(x_{0}\right)\right]$ então $\frac{f\left(x_{0}\right)-f(x)}{x_{0}-x} \leq m$.

Logo, em ambos os casos,

$$
f(x) \geq f\left(x_{0}\right)+m\left(x-x_{0}\right)
$$

e portanto $s(x)=f\left(x_{0}\right)+m\left(x-x_{0}\right)$ é uma reta-suporte para $f$ em $x_{0}$.

Reciprocamente, dado $\left.x_{0} \in[a, b] \subset J, x_{0}=\lambda a+(1-\lambda) b, \lambda \in\right] 0,1[$ se $f$ admite uma reta-suporte em $x_{0} \in J$ então existe $s(x)=f\left(x_{0}\right)+m\left(x-x_{0}\right)$ tal que $s\left(x_{0}\right)=f\left(x_{0}\right)$ e $f(x) \geq s(x)$ para todo $x \in[a, b], x \neq x_{0}$. Como

$$
\begin{aligned}
\lambda s(a)+(1-\lambda) s(b) & =\lambda f\left(x_{0}\right)+\lambda m\left(a-x_{0}\right)+(1-\lambda) f\left(x_{0}\right)+(1-\lambda) m\left(b-x_{0}\right) \\
& =f\left(x_{0}\right)+m\left(\lambda a+\left(b-x_{0}\right)-\lambda b\right)=s(\lambda a+(1-\lambda) b) \\
s\left(x_{0}\right)=f\left(x_{0}\right) & =s(\lambda a+(1-\lambda) b)=\lambda s(a)+(1-\lambda) s(b) \leq \lambda f(a)+(1-\lambda) f(b),
\end{aligned}
$$

$f$ é convexa em $[a, b]$ e portanto, em $I$.

Corolário 2.15. Se $f: I \rightarrow \mathbb{R}$ admite segunda derivada em todos os pontos então, $f$ é convexa se e somente se $f^{\prime \prime}(x) \geq 0$ para todo $x \in I$.

Prova. Se $f: I \rightarrow \mathbb{R}$ é convexa, pela Proposição $2.9, f^{\prime}$ é não-decrescente em $I$, e portanto $f^{\prime \prime}(x) \geq 0$ para todo $x \in I$.

Suponha agora que existe $\left.x_{0} \in\right] a, b\left[\right.$ tal que $f^{\prime \prime}\left(x_{0}\right)<0$. Pela fórmula de Taylor com resto de Lagrange para $x_{0}<x$,

$f(x)=f\left(x_{0}\right)+f^{\prime}\left(x_{0}\right)\left(x-x_{0}\right)+f^{\prime \prime}\left(x_{0}\right)\left(x-x_{0}\right)^{2}$.

Então $f(x)<f\left(x_{0}\right)+f^{\prime}\left(x_{0}\right)\left(x-x_{0}\right)$, e o gráfico de $f$ estaria abaixo da reta $s(x)=f\left(x_{0}\right)+f^{\prime}\left(x_{0}\right)\left(x-x_{0}\right)$. Pelo Corolário 2.14 conclui-se que $f$ não seria convexa em $] a, b[$.

OBSERVAÇÃO 2.16. Se $f^{\prime \prime}(x)>0$ para todo $x \in I$ então $f$ é estritamente convexa. Mas a recíproca não é verdadeira, pois por exemplo $f(x)=x^{4}$ é estritamente convexa mas $f^{\prime \prime}(0)=0$.

Geometricamente, ocorre que, em um ponto intermediário do segmento $\overline{x y}$, $x_{0}=\lambda x+(1-\lambda) y$ para algum $\left.\lambda \in\right] 0,1\left[\right.$, o valor de uma função convexa, $f\left(x_{0}\right)$, é tal que o ponto $\left(x_{0}, f\left(x_{0}\right)\right)$ fica abaixo do ponto correspondente no segmento que une os pontos do gráfico de $f:(x, f(x))$ e $(y, f(y))$, pela Definição 2.2 e como pode ser constatado nos seguintes exemplos de funções convexas definidas em $\mathbb{R}$. 
EXEMPLOS 2.17. Seja $f: \mathbb{R} \rightarrow \mathbb{R}$.

(1) $f(x)=a x+b$ é convexa, mas não é estritamente convexa pois $f^{\prime \prime}(x)=0$ para todo $x \in \mathbb{R}$.

(2) $f(x)=a_{0}+a_{1} x+\cdots+a_{n} x^{n}, a_{j}>0$ é estritamente convexa em $\mathbb{R}^{+}$:

$$
f^{\prime \prime}(x)=2 a_{2}+6 a_{3} x+\cdots+n(n-1) a_{n} x^{n-2}>0
$$

para todo $x \in \mathbb{R}^{+}$.

(3) $f(x)=e^{x}$ é estritamente convexa pois $f^{\prime \prime}(x)>0$ para todo $x \in \mathbb{R}$.

(4) $f(x)=|x|$ é convexa pois para todo $\lambda \in[0,1]$ :

$$
f(\lambda x+(1-\lambda) y)=|\lambda x+(1-\lambda) y| \leq \lambda f(x)+(1-\lambda) f(y) .
$$

DEFINIÇÃo 2.18. Um subconjunto $U$ de um espaço vetorial $V$ é dito convexo se $\lambda x+(1-\lambda) y \in U$ para todo par $x, y \in U$ e para todo $\lambda \in[0,1]$.

Uma função $f: U \subset V \rightarrow \mathbb{R}$ é dita convexa em $U$ se para todo par $x, y \in U$ $f(\lambda x+(1-\lambda) y) \leq \lambda f(x)+(1-\lambda) f(y)$ para todo $\lambda \in[0,1]$.

$A$ função $f$ é estritamente convexa em $U$ se esta desigualdade for estrita para todo $\lambda \in] 0,1[$.

Todo espaço vetorial normado admite funções convexas não-constantes.

EXEMPLOS 2.19. Sejam $x, y \in V, \lambda \in[0,1]$.

(1) A função norma $\|\|:. V \rightarrow \mathbb{R}$ de um espaço vetorial é uma função convexa. De fato:

$$
\|\lambda x+(1-\lambda) y\| \leq\|\lambda x\|+\|(1-\lambda) y\|=\lambda\|x\|+(1-\lambda)\|y\| .
$$

(2) A função distância a um ponto p fixado de um espaço vetorial normado $d: V \rightarrow \mathbb{R}$ definida por $d(x)=\operatorname{dist}(x, p)=\|x-p\|$ é uma função convexa.

De fato:

$$
\begin{aligned}
& d(\lambda x+(1-\lambda) y)=\|\lambda x+(1-\lambda) y-p\| \\
& =\|\lambda(x-p)+(1-\lambda)(y-p)\| \leq|\lambda|\|x-p\|+|1-\lambda|\|y-p\| \\
& =\lambda d(x)+(1-\lambda) d(y) .
\end{aligned}
$$

(3) Em particular, a função distância à origem no $\mathbb{R}^{n}$ é convexa

$$
\operatorname{dist}(x, 0)=\|x\|=\sqrt{\sum_{i=1}^{n}\left(x_{i}\right)^{2}},
$$

e a função dist ${ }^{2}(x, 0)=\|x\|^{2}$ é estritamente convexa. 
Destaca-se ainda as seguintes importantes propriedades de funções convexas definidas em $\mathbb{R}$.

LEMA 2.20. Uma função estritamente convexa, diferenciável $f: \mathbb{R} \rightarrow \mathbb{R}$ é ilimitada superiormente.

Prova. Como $f$ é estritamente convexa, existem $t_{0}<t_{1}$ tal que $f\left(t_{0}\right) \neq f\left(t_{1}\right)$. Suponha $f\left(t_{1}\right)>f\left(t_{0}\right)$. Então existe $\tau \in\left[t_{0}, t_{1}\right]$ tal que $f^{\prime}(\tau)>0$.

Como $f$ é convexa, pela Proposição $2.9, f^{\prime}(t) \geq f^{\prime}(\tau)>0$ para todo $t \geq \tau$. E também, pelo Corolário 2.14, $f(t) \geq f^{\prime}(\tau)(t-\tau)+f(\tau)$ para todo $t>\tau$. Segue que $\lim _{t \rightarrow+\infty} f(t)=+\infty$.

Se $f\left(t_{1}\right)<f\left(t_{0}\right)$, analogamente, existe $\tau \in\left[t_{0}, t_{1}\right]$ tal que $f^{\prime}(\tau)<0$.

Como $f^{\prime}$ é não-decrescente em $\mathbb{R}, f^{\prime}(t) \leq f^{\prime}(\tau)<0$ para todo $t \leq \tau$. E também, $f(t) \geq f^{\prime}(\tau)(t-\tau)+f(\tau)$ para todo $t<\tau$. Então $\lim _{t \rightarrow-\infty} f(t)=+\infty$.

Portanto, em ambos os casos, $f$ é ilimitada superiormente.

LEMA 2.21. Uma função convexa, diferenciável, $f: I \rightarrow \mathbb{R}$ que tenha dois pontos críticos a e $b, a<b$ em $I$, deve ser constante em $[a, b]$.

PROVA. Como $a$ e $b$ são pontos críticos, $f^{\prime}(a)=f^{\prime}(b)=0$.

Como a derivada $f^{\prime}$ é uma função não-decrescente, $0=f^{\prime}(a) \leq f^{\prime}(x) \leq f^{\prime}(b)=0$ para todo $x \in[a, b]$. Logo, $f^{\prime}(x)=0$ para todo $x \in[a, b]$. Portanto, $f(x)$ é constante $\mathrm{em}[a, b]$.

LEMA 2.22. Uma função estritamente convexa $f:[a, b] \rightarrow \mathbb{R}$ assume valor máximo somente em a elou em b, ou seja, nos extremos do intervalo de definição.

Prova. Seja $x=\lambda a+(1-\lambda) b, \lambda \in[0,1]$. Como $f$ é estritamente convexa,

$$
\begin{aligned}
f(x) & <\lambda f(a)+(1-\lambda) f(b) \\
& \leq f\left(x_{0}\right)+(1-\lambda) f\left(x_{0}\right)=f\left(x_{0}\right)
\end{aligned}
$$

onde $f\left(x_{0}\right)=\max \{f(a), f(b)\}$. Logo, $f(x)<f\left(x_{0}\right)$ para todo $\left.x \in\right] a, b[$. Portanto, $f$ tem ponto de máximo em $\{a, b\}$.

LEMA 2.23. Todo ponto crítico de uma função convexa $f: I \rightarrow \mathbb{R}$ não-constante é um ponto de mínimo global.

PROVA. Se $a$ é um ponto crítico de $f$, então $f^{\prime}(a)=0$.

Pelo Corolário 2.14, $f(t) \geq f(a)+f^{\prime}(a)(t-a)$, para todo $t \in I, t \neq a$.

Logo $f(t) \geq f(a)$, para todo $t \in I$ e portanto $a$ é um ponto de mínimo global. 
Assim, por estes resultados, se uma função convexa $f$, não-constante, tiver um ponto crítico no interior de $I$, este será um ponto de mínimo global e também será o único ponto crítico de $f$.

PRoposição 2.24. Se $f: I \rightarrow \mathbb{R}$ é convexa então $f$ é Lipschitz ou seja, existe $K>0$ tal que $|f(x)-f(y)|<K|x-y|$ para todo $x, y \in I$.

Prova. Sejam $x, y \in[a, b] \subset I$ com $x<y$. Seja $\epsilon>0$ tal que $[a-\epsilon, b+\epsilon]$ está contido no interior de $I$. Tome $z=y+\epsilon$. Logo, $y=\lambda z+(1-\lambda) x$ para $\lambda=\frac{y-x}{(y+\epsilon)-x} \in(0,1)$. Como $f$ é convexa:

$$
\begin{aligned}
f(y) & \leq \lambda f(z)+(1-\lambda) f(x) \\
f(y)-f(x) & \leq \lambda(f(z)-f(x))=\frac{y-x}{y-x+\epsilon}(f(z)-f(x)) \\
& <\frac{y-x}{\epsilon}(f(z)-f(x))<\frac{(M-m)}{\epsilon} y-x .
\end{aligned}
$$

onde $m$ e $M$ são limitantes inferior e superior de $f$ em $[a-\epsilon, b+\epsilon]$, respectivamente. Portanto, $f$ é Lipschitz com $K=\frac{(M-m)}{\epsilon}$.

\section{Funções convexas em variedades Riemannianas}

DEFINIÇño 2.25. Seja $(M, g)$ uma variedade Riemanniana. Um subconjunto aberto $B \subset M$ é dito convexo ${ }^{1}$ se para todo par de pontos $p, q \in B$ existe uma única geodésica minimizante em $M$ ligando $p$ a q e inteiramente contida em $B$.

Quando $M=\mathbb{R}^{n}$, esta é a bem conhecida definição (2.1) de conjuntos convexos.

No caso geral para garantir a existência de pelo menos uma geodésica ligando quaisquer pontos em $M$, precisamos assumir $(M, g)$ uma variedade Riemanniana completa, e logo geodesicamente conexa pelo Teorema de Hopf-Rinow (1.56). Assim, daqui em diante, $(M, g)$ denotará uma variedade Riemanniana completa.

Mas, diferentemente do que ocorre em espaços euclidianos com a métrica usual, podem existir outras geodésicas ligando $p$ a $q$ em $B$ convexo, mas que não permanecem em $B$. Como as propriedades geométricas das funções convexas definidas em $M$ requerem que isto não aconteça, quando não há unicidade de geodésicas é necessário adotar-se a seguinte noção de convexidade:

DEFINIÇÃO 2.26. Um subconjunto $A \subset M$ é dito totalmente convexo se contém todo segmento de geodésica entre quaisquer dois de seus pontos.

Como foi visto no Teorema 1.33, cada ponto $p \in M$ possui uma vizinhança normal isto é, uma vizinhança aberta $W$ e um número $\epsilon^{\prime}>0$ tais que quaisquer dois pontos de $W \subset B\left(p, \epsilon^{\prime}\right)$ podem ser ligados por uma geodésica minimizante de

\footnotetext{
${ }^{1}$ dito fortemente convexo em Carmo [3, Capítulo 3 - seção 4].
} 
comprimento menor que $\epsilon^{\prime}$. Pode acontecer, no entanto, que esta geodésica não esteja inteiramente contida em $W$ e portanto $W$ não será convexa.

É claro que se $W$ estiver contido em $A$, um subconjunto totalmente convexo de $M$, então $W$ será uma vizinhança normal convexa. Caso contrário, é possível escolher $r>0$ tal que $\mathcal{B}(p, r) \subset W$ se torne uma vizinhança normal convexa de $p$.

LeMA 2.27. Para cada $p \in M$ existe um número $r>0$ tal que a bola geodésica $\mathcal{B}(p, r)$ contida em uma vizinhança normal de $p$ é convexa.

$\mathcal{B}(p, r)$ é dita a bola normal em $p$ de raio $r . \quad$ ([19, Capitulo 3 - Teorema 1.2])

DEFINIÇÃo 2.28. Uma função $F: A \subset M \rightarrow \mathbb{R}$ definida em um subconjunto A de $M$ totalmente convexo, é dita convexa se para toda geodésica $\gamma:[a, b] \rightarrow A, a$ aplicação composta $F \circ \gamma:[a, b] \rightarrow \mathbb{R}$ é convexa em $[a, b]$.

Uma função $F: A \subset M \rightarrow \mathbb{R}$ é dita estritamente convexa se Fo $\gamma$ é estritamente convexa para toda geodésica não-constante em $A$.

Observe que a existência de funções convexas em $M$ depende crucialmente da métrica que determina as geodésicas em $M$. Uma função pode ser convexa em $\left(M, g_{1}\right)$ mas não o ser em $\left(M, g_{2}\right)$, com $g_{2} \neq g_{1}$.

LEMA 2.29. Uma função $F: A \subset M \rightarrow \mathbb{R}$ é convexa se e somente se

$$
F(\gamma(t)) \leq(1-t) F(p)+t F(q)
$$

para todo $p, q \in A$, para toda geodésica $\gamma:[0,1] \rightarrow$ A ligando p a q e todo $t \in[0,1]$.

Prova. Sejam $p, q \in A$ e $\gamma:[0,1] \rightarrow A$. Por definição (2.28), se $F$ é convexa em $A, f=F \circ \gamma$ é convexa em $[0,1]$ e segue, pela Definição 2.2 , que para todo par $x, y \in[0,1], s \in[x, y]$, dado por $s=(1-t) x+t y \operatorname{com} t \in[0,1]$ ocorre que

$$
f(s) \leq(1-t) f(x)+t f(y) .
$$

Em particular, para $x=0$ e $y=1$ vale que: $f(t) \leq(1-t) f(0)+t f(1)$ e portanto

$$
F(\gamma(t)) \leq(1-t) F(p)+t F(q) .
$$

Considere agora a restrição de $\gamma:[0,1] \rightarrow A$ a $[x, y] \subset[0,1]$ ligando os pontos $\gamma(x)$ e $\gamma(y)$. Tomando uma reparametrização desta restrição, $\alpha=\gamma \circ \phi$ onde

$$
\begin{aligned}
\phi:[0,1] & \rightarrow[x, y] \\
t & \rightarrow \phi(t)=x+(y-x) t=(1-t) x+t y
\end{aligned}
$$

obtem-se que: $\alpha(t)=\gamma((1-t) x+t y), t \in[0,1]$.

Como, reciprocamente, $F(\alpha(t)) \leq(1-t) F(\alpha(0))+t F(\alpha(1))$ ou seja,

$$
F(\gamma((1-t) x+t y)) \leq(1-t) F(\gamma(x))+t F(\gamma(y))
$$

$f=F \circ \gamma:[0,1] \rightarrow \mathbb{R}$ é convexa em $[0,1]$ e portanto $F$ é convexa em $A$. 
LEMA 2.30. Seja $F: A \subset M \rightarrow \mathbb{R}$ uma função convexa em $A$.

Para $c \in \mathbb{R}$, denota-se por $F^{c}$ o c-subnível fechado de $\mathrm{F}$

$$
\left.\left.F^{c}=F^{-1}(]-\infty, c\right]\right)=\{x \in M: F(x) \leq c\} .
$$

Para todo $c \in \mathbb{R}$, o subnível $F^{c}$ é um subconjunto totalmente convexo. Em particular $F^{c}$ é conexo.

Prova. Sejam $p$ e $q$ dois pontos de $A$ satisfazendo $F(p) \leq c$ e $F(q) \leq c$, e $\gamma(t)=x$ um ponto arbitrário na geodésica ligando $p$ a $q$.

Como $F$ é convexa, tem-se, pelo Lema 2.29, que

$$
F(x)=F(\gamma(t)) \leq(1-t) F(p)+t F(q) \leq(1-t) c+t c=c
$$

Então, todo ponto $x=\gamma(t)$ satisfaz $F(x) \leq c$ quando $p$ e $q$ satisfazem esta desigualdade, ou seja $\gamma(t) \in F^{c}$ quando $p, q \in F^{c}$. Portanto, pela Definição 2.26, $F^{c}$ é totalmente convexo.

Para funções de classe $C^{1}$ ou $C^{2}$ em $M$, a noção de convexidade pode ser dada em termos do gradiente e do hessiano de $F, \nabla F$ e Hess $F$ respectivamente, definidos na Seção 2 do Capítulo 1.

PRoposição 2.31. Uma função $C^{1}, F: A \rightarrow \mathbb{R}$ é convexa se e somente se para todo $p, q \in A$ e todo segmento de geodésica $\gamma:[a, b] \rightarrow$ A ligando $p$ e $q$, temos que:

$$
F(q) \geq F(p)+g\left(\nabla F_{p}, \dot{\gamma}(a)\right) .
$$

Além disso, F é estritamente convexa se a desigualdade é estrita, quando $p \neq q$.

Prova. Sejam $p, q \in A$.

Como $M$ é completa, existe $\gamma_{p q}$ um segmento geodésico em $A$ ligando $p$ a $q$.

Considere $\gamma_{p q}$ definida em $[0,1]$ por uma reparametrização adequada (Lema 1.29). Seja $f=F \circ \gamma_{p q}:[0,1] \rightarrow M$ convexa, pois $F$ o é. Como $F$ é diferenciável em $p$, pelo Corolário 2.14, para todo $t \in[0,1]$

$$
f(t) \geq f(0)+f^{\prime}(0)
$$

Como $f^{\prime}(0)=\left.\frac{d}{d t}\left(F \circ \gamma_{p q}\right)(t)\right|_{t=0}=g\left(\nabla F_{p}, \dot{\gamma}_{p q}(0)\right)$, em particular para $t=1$ :

$$
F(q)=f(1) \geq F(p)+g\left(\nabla F_{p}, \dot{\gamma}_{p q}(0)\right) .
$$

Suponha agora que para cada $p \in A, F(q) \geq F(p)+g\left(\nabla F_{p}, \dot{\gamma}_{p q}(a)\right)$ para todo $q \in A$ e todo $\gamma_{p q}$, definido em $[a, b]$. Então, para uma reparametrização conveniente de $\gamma$ em $[0,1]$, vale que:

$$
F(q) \geq F(p)+g\left(\nabla F_{p}, \dot{\gamma}_{p q}(0)\right)
$$


Tomando-se $\gamma_{q p}(t)=\gamma_{p q}(1-t), t \in[0,1]$ um segmento geodésico ligando $q$ a $p$, também vale que:

$$
F(p) \geq F(q)+g\left(\nabla F_{q}, \dot{\gamma}_{q p}(0)\right)
$$

Fixe $t$ em $[0,1]$ e tome a restrição de $\gamma_{p q}$ tal que $\gamma_{p q}(u), u \in[t, 1]$ é um segmento geodésico ligando $\gamma_{p q}(t)$ a $q$ ou seja, considere uma reparametrização de $\gamma_{p q}$ dada por $\alpha_{t}=\gamma_{p q} \circ \sigma_{t}:[0,1] \rightarrow A$ onde

$$
\begin{aligned}
& \sigma_{t}=\sigma:[0,1] \rightarrow[t, 1] \\
& s \rightarrow u=\sigma(s)=(1-s) t+s=t+s(1-t) \\
& \alpha_{t}(s)=\alpha(s)=\gamma_{p q}(\sigma(s))=\gamma_{p q}(t+s(1-t)) \\
& \alpha(0)=\gamma_{p q}(t) \\
& \frac{d \alpha}{d s}(0)=(1-t) \dot{\gamma}_{p q}(t) .
\end{aligned}
$$

A restrição $\gamma_{q p}(r)=\gamma_{p q}(1-r), r \in[1-t, 1]$ é um segmento geodésico ligando $\gamma_{p q}(t)\left(=\gamma_{q p}(1-t)\right)$ a $p\left(=\gamma_{q p}(1)\right)$ ou seja, tome $\beta=\gamma_{q p} \circ \rho:[0,1] \rightarrow A$ onde

$$
\begin{aligned}
\rho:[0,1] \rightarrow[ & {[1-t, 1] } \\
s \rightarrow r & =\rho(s)=(1-s)(1-t)+s=1-t+s t \\
\beta(s) & =\gamma_{q p}(\rho(s))=\gamma_{q p}(r)=\gamma_{p q}(t-s t) \\
\beta(0) & =\gamma_{q p}(1-t)=\gamma_{p q}(t) \\
\frac{d \beta}{d s}(0) & =-t \dot{\gamma}_{p q}(t) .
\end{aligned}
$$

(2.1) é verdadeira se substituimos $p$ por $\gamma_{p q}(t)=\alpha(0)$ e $\dot{\gamma}_{p q}(0)$ por $\frac{d \alpha}{d s}(0)$ obtendo:

$$
F(q) \geq F\left(\gamma_{p q}(t)\right)+(1-t) g\left(\nabla F\left(\gamma_{p q}(t)\right), \dot{\gamma}_{p q}(t)\right)
$$

Analogamente, em (2.2) substitui-se $q$ por $\gamma_{p q}(t)=\beta(0)$ e $\dot{\gamma}_{q p}(0)$ por $\frac{d \beta}{d s}(0)$ obtendo:

$$
F(p) \geq F\left(\gamma_{p q}(t)\right)+-t g\left(\nabla F\left(\gamma_{p q}(t)\right), \dot{\gamma}_{p q}(t)\right)
$$

Multiplicando (2.3) por $t$ e (2.4) por $(1-t)$, e somando, obtem-se:

$$
t F(q)+(1-t) F(p) \geq F\left(\gamma_{p q}(t)\right) .
$$

Como $t$ é arbitrário em [0,1], pelo Lema 2.29, conclui-se que $F$ é convexa em $A$.

Analogamente ao caso real (Proposição 2.7), se $F$ é convexa existem as chamadas derivadas direcionais unilaterais de F em p numa dada direção $v$, conforme a teoria desenvolvida em Udriste [19, Seção 3.4 - Derivadas direcionais e subgradientes]. Para estas derivadas unilaterais também vale uma propriedade análoga à da Proposição 2.31, dada no Teorema 4.10 . 
PRoposiç̃̃o 2.32. Uma função $C^{2}, F: A \rightarrow \mathbb{R}$ é convexa se e somente se seu hessiano Hess $F$ é semidefinido positivo em $A$.

Prova. Sejam $p \in A$ e $v \in T_{p} M$. Considere $\left.\gamma:\right]-\delta, \delta[\rightarrow A$ a única geodésica passando por $p=\gamma(0) \operatorname{com} \dot{\gamma}(0)=v$.

Como $f=F$ o $\gamma$ é convexa em ] $-\delta, \delta$ [ pois $F$ o é, pelo Corolário $2.15, f^{\prime \prime}(t) \geq 0$ para todo $t \in]-\delta, \delta\left[\right.$. Em particular $f^{\prime \prime}(0) \geq 0$. Segue, pelo Lema 1.23, que

$$
f^{\prime \prime}(0)=\left.\frac{d^{2}}{d t^{2}}(F \circ \gamma)(t)\right|_{t=0}=\operatorname{Hess} F(p)(v, v) \geq 0 .
$$

Portanto Hess $F$ é semidefinido positivo em A, pois $v$ e $p$ são arbitrários.

Seja $F: A \rightarrow \mathbb{R}$ de classe $C^{2}$ e suponha que Hess $F(p)(v, v) \geq 0$ para todo $v \in T_{p} M$ e todo $p \in A$. Fixemos $p$ em $A$. Então, tomando $\left.\gamma:\right]-\delta, \delta[\rightarrow A$ uma geodésica qualquer passando por $p=\gamma(0)$, para cada $t \in]-\delta, \delta[$ vale que Hess $F(\gamma(t))[\dot{\gamma}(t), \dot{\gamma}(t)] \geq 0$. Pelo Lema 1.23, obtem-se que

$$
\text { Hess } F(\gamma(t))[\dot{\gamma}(t), \dot{\gamma}(t)]=\frac{d^{2}}{d t^{2}}(F \circ \gamma)(t)=f^{\prime \prime}(t) \geq 0
$$

onde $f=F \circ \gamma$. Segue, pelo Corolário 2.15, que $f$ é convexa em $]-\delta, \delta[$. Como, para cada $p \in A, \gamma$ é uma geodésica arbitrária em $A, f=F \circ \gamma:]-\delta, \delta[\rightarrow \mathbb{R}$ é convexa em ] $-\delta, \delta[$ para toda geodésica passando por $p=\gamma(0)$. Portanto, por definição, $F$ é convexa $\mathrm{em} A$.

OBSERVAÇÃo 2.33. Se Hess $F$ é definido positivo exceto em um conjunto discreto então $F$ é estritamente convexa.

DEFINIÇão 2.34. Uma função de classe $C^{2}, F: M \rightarrow \mathbb{R}$, é dita fortemente convexa se existe uma função (contínua) $\lambda: M \rightarrow \mathbb{R}^{+}$tal que

$$
\text { Hess } F(p)(v, v) \geq \lambda(p) g(v, v)
$$

para todo $p \in M$, e para todo $v \in T_{p} M$.

Se $F$ é fortemente convexa, Hess $F(p)$ é definido positivo em $M$, exceto talvez num conjunto discreto: os zeros de $\lambda$, e então, $F$ é estritamente convexa.

Em particular, se $K \subset M$ é compacto, $\left.\lambda\right|_{K}$ assume um valor máximo $\lambda_{0}>0 \mathrm{em} K$ tal que Hess $F(p)$ é definido positivo em $K$ e logo $\left.F\right|_{K}$ é estritamente convexa.

Mas a recíproca não vale. Por exemplo, $f(x)=\mathrm{e}^{x}$ é estritamenta convexa em $\mathbb{R}$ mas $f$ não é fortemente convexa pois não existe um número $m>0$ tal que $f^{\prime \prime}(x)=\mathrm{e}^{x} \geq m>0$ já que inf ${ }_{x}\left\{\mathrm{e}^{x}\right\}=0$.

As propriedades de continuidade, dadas nas Proposições 2.5 e 2.24, são a seguir estendidas para funções convexas definidas em $(M, g): F$ é contínua em todo ponto interior de $A$, subconjunto totalmente convexo de $M$, e além disso, $F$ é Lipschitz 
em todo subconjunto compacto de $M$. Assim, a maioria dos resultados envolvendo funções convexas não pressupõem nenhuma regularidade sobre elas.

Proposição 2.35. Se $F: A \subset M \rightarrow \mathbb{R}$ é convexa em A então $F$ é contínua em todo ponto interior de A.

Prova. Seja $p_{0} \in \operatorname{int}(A)$. Escolha $c \in \mathbb{R}$ tal que o conjunto totalmente convexo $F^{c}=\{x \in M: F(x) \leq c\}$ (Lema 2.30) contenha $\mathcal{B}\left(p_{0}, r\right)$, com $r$ suficientemente pequeno.

Seja $\gamma:[-1,1] \rightarrow A$ uma geodésica em $\overline{\mathcal{B}\left(p_{0}, r\right)}$ tal que $\gamma(-1)=p_{1}, \gamma(0)=p_{0}$ e $\gamma(1)=p_{2}$, e parametrizada proporcionalmente à distância $\operatorname{dist}\left(p_{0}, p\right)$ ou seja, faça $\gamma(t)=p$ onde

$$
t=\frac{\operatorname{dist}\left(p_{0}, p\right)}{r} \in[0,1] .
$$

Como $F$ é convexa tem-se, pelo Lema 2.29, que:

$$
F(\gamma(t)) \leq(1-t) F\left(p_{0}\right)+t F\left(p_{2}\right) \leq(1-t) F\left(p_{0}\right)+t c
$$

para todo $t \in[0,1]$ isto é

$$
F(p)-F\left(p_{0}\right) \leq t\left(c-F\left(p_{0}\right)\right) .
$$

$\mathrm{O}$ arco de geodésica ligando $p_{1}$ e $p$ é a restrição $\gamma(u), u \in[-1, t]$. Fazendo $u=-1+s(t+1), s \in[0,1]$ obtem-se a reparametrização: $\alpha(s)=\gamma(-1+s(t+1))$ $\operatorname{com} \alpha(0)=\gamma(-1)=p_{1}, \alpha\left(\frac{1}{1+t}\right)=\gamma(0)=p_{0}$ e $\alpha(1)=\gamma(t)=p$.

Devido à convexidade de $F, F \circ \alpha$ é convexa ou seja,

$$
F(\alpha(s)) \leq(1-s) F\left(p_{1}\right)+s F(p) \leq(1-s) c+s F(p) .
$$

Segue que, para $s=\frac{1}{1+t}$

$$
F\left(p_{0}\right) \leq \frac{t}{1+t} c+\frac{1}{1+t} F(p) \Longrightarrow F\left(p_{0}\right) \leq t c-t F\left(p_{0}\right)+F(p)
$$

ou seja,

$$
F(p)-F\left(p_{0}\right) \geq-t\left(c-F\left(p_{0}\right)\right)
$$

Das expressões (2.5) e (2.6), obtem-se que:

$$
\left|F(p)-F\left(p_{0}\right)\right| \leq t\left(c-F\left(p_{0}\right)\right) .
$$

Como $t=\frac{\operatorname{dist}\left(p_{0}, p\right)}{r}$, segue que $\lim _{p \rightarrow p_{0}} F(p)=F\left(p_{0}\right)$.

Proposição 2.36. Seja $(M, g)$ uma variedade Riemanniana completa. Se $F: M \rightarrow \mathbb{R}$ é convexa e limitada superiormente então $F$ é constante em $M$. 
Prova. Suponha que $F$ é uma função convexa não-constante em $M$. Então, por definição, existe $\gamma: \mathbb{R} \rightarrow M$ uma geodésica não-constante em $M$ completa, tal que $F \circ \gamma$ é convexa e não-constante em $\mathbb{R}$. Pelo Lema 2.20, $F$ o $\gamma$ é ilimitada ao infinito. E portanto $F$ também o é em $M$.

COROLÁrio 2.37. Se $M$ possui uma geodésica fechada isto é, se $\gamma: \mathbb{R} \rightarrow M$ é uma geodésica não-constante periódica em $M$, então $F: M \rightarrow \mathbb{R}$ convexa, deve ser constante ao longo de $\gamma$.

Prova. Seja $\gamma: \mathbb{R} \rightarrow M$ uma geodésica fechada em $M$. Como $F$ é convexa, pela Proposição 2.35, $F$ é contínua em todo ponto interior de $M$ e logo $\left.F\right|_{\gamma(\mathbb{R})}$ é contínua no compacto $\gamma(\mathbb{R}) \subset M$. Então $F$ assume valores máximo e mínimo em $\gamma(\mathbb{R})$ e assim, $F$ é limitada superiormente em $\gamma(\mathbb{R})$. Portanto, pela Proposição 2.36, $F$ é constante ao longo de $\gamma$.

A existência de funções convexas em $(M, g)$ não depende exclusivamente da métrica $g$ em $M$, mas também da topologia de $M$. Decorre da Proposição 2.36 que, em particular, variedades Riemannianas compactas não admitem funções convexas não-constantes. Mais geralmente, se uma variedade Riemanniana completa $(M, g)$ admite uma função convexa não-constante então tem volume infinito. Este resultado foi mostrado por Bishop ([1, Proposição 2.2]) em 1969 para funções de classe $C^{2}$. Esta é a única, por enquanto conhecida, consequência da existência de uma função convexa não-constante na estrutura métrica da variedade.

É claro que, existem variedades Riemannianas não completas com volume finito que admitem funções convexas não-constantes. Este é o caso de $f: \mathbb{R}^{n} \rightarrow \mathbb{R}$ definida por $f(x)=\|x\|^{2}$, dada no Exemplo 2.19, e tomando-se sua restrição à bola unitária $\mathcal{B}(0,1)$ em $\mathbb{R}^{n}$ com a métrica euclidiana induzida.

Assim, somente a existência de uma função convexa não-constante não tem implicações na estrutura topológica da variedade exceto quanto às propriedades de nãocompacidade. Sabe-se, por um resultado de Green e Shiohama [8], que em toda variedade não-compacta $M$ existem uma métrica Riemanniana completa $g$ em $M$ e uma função não-constante $C^{\infty}, F: M \rightarrow \mathbb{R}$ que é convexa relativamente a $g$.

No entanto, a existência de uma função convexa não-constante satisfazendo às propriedades dadas a seguir, como por exemplo admitindo um ponto de mínimo isolado, ou tal que seus subníveis são compactos, implica em substancial informação sobre a estrutura topológica e métrica da variedade. Por exemplo, será mostrado que se $(M, g)$ admite uma função convexa que tem um ponto de mínimo não-degenerado então $M$ é contrátil. Além disso, sob as mesmas condições, $M$ é difeomorfa a $\mathbb{R}^{m}$. 
Proposição 2.38. Seja $F: M \rightarrow \mathbb{R}$ uma função convexa, duas vezes diferenciável. Se F tem um ponto crítico não-degenerado $p_{0}$ então $p_{0}$ é um ponto de mínimo global de F e êle é o único ponto crítico de F.

Prova. Sejam $q \in M, q \neq p_{0}$ e $\gamma:[a, b] \rightarrow M$, uma geodésica qualquer não-constante ligando $p_{0}=\gamma(a)$ a $q=\gamma(b)$.

Por definição, $f=F \circ \gamma:[a, b] \rightarrow \mathbb{R}$ é convexa em $[a, b]$.

Como $p_{0}$ é ponto crítico de $F$, e $f^{\prime}(a)=g(\nabla F(\gamma(a)), \dot{\gamma}(a))=g\left(\nabla F\left(p_{0}\right), \dot{\gamma}(a)\right)=0$ segue que $a$ é ponto crítico de $f$.

Além disso, $f^{\prime \prime}(s)=\frac{d}{d s} g(\nabla F(\gamma(s)), \dot{\gamma}(s))=g\left(\right.$ hess $\left.F(\gamma(s))_{\dot{\gamma}(s)}, \dot{\gamma}(s)\right)$ e logo, $f^{\prime \prime}(a)=g\left(\right.$ hess $\left.F\left(p_{0}\right)_{\dot{\gamma}(a)}, \dot{\gamma}(a)\right)$.

Como $p_{0}$ é ponto crítico não-degenerado de $F$, o operador hess $F\left(p_{0}\right)$ é invertível ou seja, hess $F\left(p_{0}\right)_{v} \neq 0$, para todo $v \in T_{p_{0}} M \backslash\{0\}$. Logo $f^{\prime \prime}(a) \neq 0$ e $a$ também é um ponto crítico não-degenerado de $f$.

Como $f$ é convexa, pelo Corolário $2.15, f^{\prime \prime}(s) \geq 0$ para todo $s \in[a, b]$, e então $f^{\prime \prime}(a)>0$ e $a$ é ponto de mínimo de $f$ (Proposição 1.28).

Logo $F\left(p_{0}\right)=f(a) \leq f(s)=F(\gamma(s))$ para todo $s \in[a, b]$. E além disso, Hess $F\left(p_{0}\right)$ é definido positivo. Portanto $p_{0}$ é ponto de mínimo global de $F$, pois $q$ é arbitrário em $M$ completa, e logo geodesicamente conexa.

Suponha que $F$ tenha outro ponto crítico $q_{0} \neq p_{0}$ e seja $\gamma:[a, b] \rightarrow M$ qualquer geodésica ligando $p_{0}$ a $q_{0}$. Então a função $f(s)=F(\gamma(s))$ tem dois pontos críticos em $a$ e $b$. Segue, pelo Lema 2.21, que $f$ é constante em $[a, b]$, e logo $\left.g\left(\text { hess } F\left(p_{0}\right)\right)_{\dot{\gamma}(a)}, \dot{\gamma}(a)\right)=f^{\prime \prime}(a)=0$, o que contradiz a hipótese de que $p_{0}$ é ponto crítico não-degenerado. Portanto $p_{0}$ é o único ponto crítico não-degenerado de $F$ e é ponto de mínimo global de $F$.

Proposição 2.39. Seja $F: M \rightarrow \mathbb{R}$ uma função convexa, duas vezes diferenciável. Se F tem um ponto crítico não-degenerado $p_{0}$ então

$$
\lim _{x \rightarrow \infty} F(x)=+\infty \text {. }
$$

Prova. Pela Proposição 2.38, $p_{0}$ é o único ponto de mínimo de $F$.

Seja $\left\{x_{n}\right\}_{n \in \mathbb{N}}$ uma sequência qualquer divergente em $M$ isto é, uma sequência que satisfaz

$$
\lim _{n \rightarrow \infty} \operatorname{dist}\left(p_{0}, x_{n}\right)=+\infty .
$$

Precisa-se mostrar que $\lim _{n \rightarrow \infty} F\left(x_{n}\right)=+\infty$.

Para tanto, seja $B$ uma vizinhança aberta, limitada, fixa de $p_{0}$, e $A$ uma vizinhança convexa de $p_{0}$ (Lema 2.27), contendo o fecho de $B$. Tomando $A$ pequena o suficiente, pode-se assumir que o fecho de $B$ e sua fronteira $\partial B=\bar{B} \backslash B$ são subconjuntos compactos em $M$. 
Para todo $x \in A$, considere $\gamma_{x}:[0, d x] \rightarrow A$ a única geodésica minimizante ligando $p_{0}$ a $x$ isto é, $\gamma_{x}(0)=p_{0}, \gamma_{x}(d x)=x$ e $\operatorname{dist}\left(p_{0}, x\right)=d x$.

E defina em $A$ a seguinte função: $\phi: A \rightarrow \mathbb{R}$ dada por

$$
\phi(x)=g\left(\nabla F(x), \dot{\gamma}_{x}(d x)\right)=\left.\frac{d}{d s} F\left(\gamma_{x}(s)\right)\right|_{s=d x} \text { e } \operatorname{com} \phi\left(p_{0}\right)=0 .
$$

Como $f=F \circ \gamma_{x}$ é convexa, $\phi(x)=f^{\prime}(d x)$ e a derivada de funções convexas é não-decrescente (Proposição 2.9), $\phi(x) \geq 0$ para $x \in A$. Seja

$$
\delta_{0}=\min _{\partial B} \phi .
$$

Então $\delta_{0}>0$. Pois, caso contrário, se $\delta_{0}=0$, existiria $x \in \partial B$ tal que $\phi(x)=0$, e a derivada de $f$ se anularia nos dois pontos extremos 0 e $d x$. Logo $f$ seria constante em $[0, d x]$, contradizendo a hipótese de que Hess $F\left(p_{0}\right)$ é uma forma não-degenerada.

Faça também

$$
f_{0}=\min _{\partial B} F>-\infty \quad \epsilon_{0}=\max _{x \in \partial B}\left\{\operatorname{dist}\left(p_{0}, x\right)\right\}<\infty .
$$

Seja $\gamma_{n}:\left[0, b_{n}\right] \rightarrow M$ a única geodésica minimizante ligando $p_{0}$ e $x_{n}$.

Pela convexidade de $A$, para todo $n \in \mathbb{N}$, existe um único $s_{n} \in\left[0, b_{n}\right]$ tal que $\gamma_{n}\left(s_{n}\right)=p_{n} \in \partial B \subset A$.

Considere $g_{n}=F \circ \gamma_{n}$ que é convexa em $\left[0, b_{n}\right]$. Pelo Corolário 2.14 e como $\operatorname{dist}\left(p_{n}, x_{n}\right) \geq \operatorname{dist}\left(p_{0}, x_{n}\right)-\operatorname{dist}\left(p_{0}, p_{n}\right) \geq \operatorname{dist}\left(p_{0}, x_{n}\right)-\epsilon_{0}$,

$$
\begin{aligned}
F\left(x_{n}\right)=g_{n}\left(b_{n}\right) & \geq g_{n}\left(s_{n}\right)+g_{n}^{\prime}\left(s_{n}\right)\left(b_{n}-s_{n}\right) \\
& =F\left(p_{n}\right)+\phi\left(\gamma_{n}\left(s_{n}\right)\right) \operatorname{dist}\left(p_{n}, x_{n}\right) \\
& \geq f_{0}+\delta_{0}\left(\operatorname{dist}\left(p_{0}, x_{n}\right)-\epsilon_{0}\right) .
\end{aligned}
$$

Então $\lim _{n \rightarrow \infty} F\left(x_{n}\right)=+\infty$. Como $\lim _{n \rightarrow-\infty} \operatorname{dist}\left(p_{0}, x_{n}\right)=+\infty$, ocorre também que $\lim _{n \rightarrow-\infty} F\left(x_{n}\right)=+\infty$. Portanto $\lim F(x)=+\infty$ tanto para $x \rightarrow-\infty$ como para $x \rightarrow+\infty$.

COROLÁrio 2.40. Seja $F: M \rightarrow \mathbb{R}$ uma função convexa não-constante, duas vezes diferenciável. Se F tem um ponto crítico não-degenerado $p_{0}$ então para todo $c \in \mathbb{R}$, o subnível $F^{c}$ é compacto em $M$.

Prova. Suponha que existe $c \in \mathbb{R}$, tal que $F^{c}=\{x \in M: F(x) \leq c\}$ é ilimitado. Então existe $\left(x_{n}\right) \subset F^{c}$ uma sequência divergente que satisfaz:

$$
\lim _{n \rightarrow \infty} \operatorname{dist}\left(p_{0}, x_{n}\right)=+\infty .
$$

Como pela Proposição 2.39, $F$ é ilimitada, $\lim _{n \rightarrow \infty} F\left(x_{n}\right)=+\infty$, o que contradiz o fato de que $F\left(x_{n}\right) \leq c$. Portanto, para todo $c \in \mathbb{R}$, o subnível $F^{c}$ é compacto em $M$ completa, pois todo subconjunto fechado e limitado em $M$, pelo Teorema de Hopf-Hinow (1.56), é compacto. 
Observe que $F^{c}=F^{-1}\left(\left[F\left(p_{0}\right), c\right]\right)$ compacto em $M$ implica que $F$ se comporta como uma função própria isto é: uma aplicação contínua tal que a imagem inversa $F^{-1}(I)$ de todo compacto $I \subset \mathbb{R}$ é um conjunto compacto.

Além disso, o significado intuitivo de uma aplicação $f: X \rightarrow Y$ ser própria é que "se x tende para a fronteira de $X$ então $f(x)$ tende para a fronteira de $Y$ ". Mais precisamente, pode-se afirmar que: Uma aplicação contínua $f: X \rightarrow Y$ é própria se e somente se a imagem $\left(f\left(x_{n}\right)\right)$ de toda sequência $\left(x_{n}\right)$ em $X$ sem subsequências convergentes é uma sequência em $Y$ que também não possui subsequências convergentes. E de fato este é o resultado dado pela Proposição 2.39.

Lembremos que: Um espaço topológico $M$ é dito contrátil se, para algum $p_{0} \in M$, a aplicação constante $p \rightarrow p_{0}$ é homotópica à aplicação identidade de $M$, ou seja, se existe uma aplicação contínua $h: M \times[a, b] \rightarrow M$ tal que $\left\{\begin{array}{l}h(p, a)=p \\ h(p, b)=p_{0}\end{array}\right.$ para todo $p \in M$.

PROPOSIÇÃo 2.41. Se M admite uma função convexa $F$ que tem um ponto de mínimo não-degenerado então $M$ é contrátil.

Prova. A idéia central é usar o fluxo do campo gradiente de F. Seja

$\eta: M \times \mathbb{R} \rightarrow M$

$$
(x, t) \rightarrow \eta(x, t)
$$

o fluxo de $-\nabla F$. Pela Definição 1.68 , para cada $x \in M$, a aplicação contínua $\gamma_{x}(t)=\eta(x, t)$ é a única curva integral de $-\nabla F$ tal que $\gamma_{x}(0)=x$.

Observe que, como $\nabla F$ é de classe $C^{1}$ (se $F$ é pelo menos $C^{2}$ ), $\nabla F$ é localmente lipschitz em $M$ e portanto o problema de Cauchy $\left\{\begin{array}{l}\dot{\gamma}(t)=\nabla F(\gamma(t)) \\ \gamma(0)=x\end{array}\right.$ tem uma única solução maximal, definida em $[0,+\infty[$.

Como $F$ possui um (único) ponto de mínimo $p_{0}$, pelo Corolário 2.40 , todo subnível $F^{c}$ de $F$ é compacto e pode-se afirmar que, para todo $x \in M$

$$
\lim _{t \rightarrow \infty} \eta(x, t)=p_{0}
$$

De fato, $h(t)=F(\eta(x, t))$ é decrescente pois:

$$
h^{\prime}(t)=g\left(\nabla F(\eta(x, t)), \dot{\gamma}_{x}(t)\right)=-g(\nabla F(\eta(x, t)), \nabla F(\eta(x, t))) \leq 0,
$$

logo $F(\eta(x, t)) \leq F(\eta(x, 0))=F(x)$ e tomando-se $c=F(x), \eta(x, t)$ está contida no subconjunto compacto $F^{c}$. Se (2.7) não ocorrer, como $F$ é contínua,

$$
\eta(x, t) \in F^{c} \backslash F^{\delta}
$$

tal que $\delta$ é o valor mínimo de $F(\eta(x, t))$ em $F^{c}$. Como $p_{0}$ é o único ponto crítico de $F, \delta$ não é valor crítico de $F$ e logo $\nabla F$ não se anula ao longo de $\eta(x, t)$. Assim,

$$
g(\nabla F(\eta(x, t)), \nabla F(\eta(x, t))) \geq \rho_{0}>0
$$


para todo $t \geq 0$. Logo, $h^{\prime}(t) \leq-\rho_{0}<0$ para todo $t \geq 0$ e então

$$
\lim _{t \rightarrow \infty} F(\eta(x, t))=-\infty
$$

Isto contradiz o fato de que $F$ tem um mínimo global em $M$ e portanto

$$
\lim _{t \rightarrow \infty} \eta(x, t)=p_{0}
$$

Denotando-se por $[0,+\infty]$ a compactificação a um ponto da semi-reta $[0,+\infty[$, homeomorfa ao intervalo compacto $[a, b]$, segue que $\eta$ pode ser estendida a uma aplicação contínua $\tilde{\eta}: M \times[0,+\infty] \rightarrow M$ fazendo-se $\tilde{\eta}(x,+\infty)=p_{0}$ e tal que a aplicação $\tilde{\eta}$ dá-nos uma homotopia entre $M$ e a singularidade $\left\{p_{0}\right\}$, provando que $M$ é contrátil.

PROPOSIÇ̃̃o 2.42. Se M admite uma função convexa $F$ que tem um ponto de mínimo não-degenerado então $M$ é difeomorfa a $\mathbb{R}^{m}$.

Prova. Seja $p_{0}$ o ponto de mínimo não-degenerado de $F$, aliás o único ponto crítico de $F$. Pela Definição 1.44, o índice Morse de $F, \lambda$, é igual a 0 . E além disso, $F^{c}$ é compacto para todo $c \in \mathbb{R}$.

Seja $U$ uma vizinhança aberta de $p_{0}$. Pelo Lema de Morse (1.27) existe um sistema de coordenadas $\left(U, \varphi=\left(x_{1}, x_{2}, \cdots, x_{m}\right)\right), \varphi: U \rightarrow \mathbb{R}^{m}$, tal que $x_{i}\left(p_{0}\right)=0$ para todo $i$, e $F$ é escrita em coordenadas como

$$
F\left(x_{1}, x_{2}, \cdots, x_{m}\right)=F\left(p_{0}\right)+\sum_{i=1}^{m} x_{i}^{2} .
$$

ou seja, para todo $q \in U$

$$
F(\varphi(q))=F\left(x_{1}(q), \cdots, x_{m}(q)\right)=F\left(p_{0}\right)+\sum_{i=1}^{m} x_{i}^{2}(q) .
$$

Seja $\epsilon>0$, escolhido pequeno o suficiente para que

$$
\overline{\mathcal{B}(0, \epsilon)}=\left\{\left(x_{1}, x_{2}, \cdots, x_{m}\right) \in \mathbb{R}^{m}: \sum_{i=1}^{m} x_{i}^{2} \leq \epsilon\right\} \subset \varphi(U) .
$$

Então o subnível $F^{\epsilon}=\{q \in M: F(q) \leq \epsilon\}$ é homeomorfo à bola fechada $\overline{\mathcal{B}(0,1)} \subset \mathbb{R}^{m}$. Seja $\phi: F^{\epsilon} \rightarrow \overline{\mathcal{B}(0,1)}$ tal homeomorfismo; $\phi$ induz um homeomorfismo entre os bordos $\partial F^{\epsilon}$ e $\partial \overline{\mathcal{B}(0,1)} \simeq S^{m-1}$, a esfera unitária em $\mathbb{R}^{m}$.

Seja $\psi(q, t)$ o fluxo do campo vetorial $X=\frac{-\nabla F}{\|\nabla F\|^{2}}$.

Denote por $\alpha(t)=\psi_{q}(t)$ a única curva integral de $X$ tal que $\alpha(0)=q, q \neq p_{0}$.

Como

$$
\frac{d}{d t} F(\alpha(t))=g(\nabla F(\alpha(t)), \dot{\alpha}(t))=-g\left(\nabla F(\alpha(t)), \frac{\nabla F(\alpha(t))}{\|\nabla F(\alpha(t))\|^{2}}\right)=-1
$$


$F$ ao longo de $\alpha$ é estritamente decrescente ou seja $F(\alpha(t))<F(\alpha(0))=F(q)$ para todo $t>0$. E também $\int_{0}^{t} \frac{d}{d r} F(\alpha(r)) d r=-t \Longrightarrow F(\alpha(t))=-t+F(q)$.

Assim, para todo $q \in M \backslash\left\{p_{0}\right\}$, a aplicação $\psi(q,)=.\alpha$ está definida em $]-\infty, F(q)[$. Defina a aplicação $H: M \backslash F^{\epsilon} \rightarrow \mathbb{R}^{m} \backslash \overline{\mathcal{B}(0,1)}$ por

$$
H(y)=\frac{1}{\epsilon} F(y) \cdot \phi(\psi(y, F(y)-\epsilon)) .
$$

A aplicação $H$ é contínua e $K: \mathbb{R}^{m} \backslash \overline{\mathcal{B}(0,1)} \rightarrow M \backslash F^{\epsilon}$ dada por

$$
K(z)=\psi\left(\phi^{-1}\left(\frac{z}{|z|}\right), \epsilon(1-|z|)\right)
$$

é a inversa contínua de $H$. Logo $H$ é um homeomorfismo.

Além disso, $H$ estende-se continuamente ao $\partial F^{\epsilon}$ fazendo-se $H(y)=\phi(y)$, para todo $y \in \partial F^{\epsilon}$. Segue que a aplicação $H: M \rightarrow \mathbb{R}^{m}$ dada por $H(y)=\left\{\begin{array}{l}\phi(y) \text { se } y \in F^{\epsilon} \\ H(y) \text { se } y \notin F^{\epsilon}\end{array}\right.$ é um homeomorfismo entre $M$ e $\mathbb{R}^{m}$.

2.1. Exemplos. A noção de convexidade tem há tempos sido associada com curvatura negativa, pois funções convexas aparecem naturalmente em variedades com curvatura não-positiva (Exemplos 2.48, 2.49), mas há inúmeros exemplos de variedades com curvatura positiva que admitem funções convexas. O Exemplo 2.44 sobre o parabolóide $z=x^{2}+y^{2}$ em $\mathbb{R}^{3}$ ilustra este fato. Na verdade, toda variedade nãocompacta com curvatura seccional não-negativa admite uma função de Busemann, definida a seguir, que é convexa (Exemplos 2.43 e 2.46). Portanto não há relação entre a curvatura de $(M, g)$ e a existência de funções convexas definidas em $M$.

Por outro lado, como a existência de funções convexas depende da métrica, pode-se estabelecer condições sobre a métrica que garantam sua existência, como mostra os Exemplos 2.50 e 2.51 .

Seja $(M, g)$ uma variedade Riemanniana completa e não-compacta.

Fixe $p_{0} \in M$. Pelo Lema 1.66 existe o raio $\gamma:\left[0,+\infty\left[\rightarrow M\right.\right.$ partindo de $p_{0}$ com $\dot{\gamma}(0)=v$, e tal que $\operatorname{dist}\left(p_{0}, \gamma(t)\right)=t$. Logo $\lim _{t \rightarrow \infty} \operatorname{dist}\left(p_{0}, \gamma(t)\right)=+\infty$.

Seja $\eta_{t}(p)=t-\operatorname{dist}(p, \gamma(t)), p \in M, t \in[0,+\infty[$.

A família de funções $\eta_{t}: M \rightarrow \mathbb{R}$ é Lipschitz, com constante $K=1$, pois $\left\|\eta_{t}\left(p_{1}\right)-\eta_{t}\left(p_{2}\right)\right\|=\left\|t-\operatorname{dist}\left(p_{1}, \gamma(t)\right)-t+\operatorname{dist}\left(p_{2}, \gamma(t)\right)\right\| \leq \operatorname{dist}\left(p_{1}, p_{2}\right) \mathrm{e}$ também satisfaz $\left\|\eta_{t}(p)\right\| \leq \operatorname{dist}\left(p, p_{0}\right)$, pela desigualdade triangular.

É portanto uma família equicontínua, uniformemente limitada em subconjuntos compactos de $M$. Segue, pelo Teorema de Ascoli-Arzelá ([9, pág. 329]), que existe uma subsequência $\left(\eta_{n}\right)$ da família $\left(\eta_{t}\right)$ que converge a uma função contínua 
$\eta: M \rightarrow \mathbb{R}$. Além disso,

$$
\begin{aligned}
\eta_{t}(p)-\eta_{s}(p) & =t-s+\operatorname{dist}(p, \gamma(s))-\operatorname{dist}(p, \gamma(t)) \\
& =\operatorname{dist}(\gamma(t), \gamma(s))-\operatorname{dist}(p, \gamma(t))+\operatorname{dist}(p, \gamma(s)) \geq 0
\end{aligned}
$$

pois $\operatorname{dist}(\gamma(t), \gamma(s))+\operatorname{dist}(\gamma(s), p) \geq \operatorname{dist}(p, \gamma(t))$ e então a família $\left(\eta_{t}\right)$ é monótona, não-decrescente. Logo, além de subsequências, o $\lim _{t \rightarrow \infty} \eta_{t}(p)=\eta(p)$ existe e é uniforme em subconjuntos compactos de $M$.

A função $\eta_{\gamma}: M \rightarrow \mathbb{R}$ dada por:

$$
\eta_{\gamma}(p)=\lim _{t \rightarrow \infty}[t-\operatorname{dist}(p, \gamma(t))]
$$

é a função de Busemann associada ao raio $\gamma$.

EXEMPLO 2.43. Se $(M, g)$ tem curvatura seccional K não-negativa (na verdade é suficiente que se tenha curvatura não-negativa fora de um subconjunto compacto de M) então cada função de Busemann $\eta_{\gamma}$ é convexa.

Se $K>0$ então a função de Busemann $\eta_{\gamma}$ é estritamente convexa.

O supremo $\eta_{p_{0}}$ de todas funções de Busemann associadas aos raios partindo de um ponto fixado $p_{0} \in M$ é uma função convexa que é não-constante em toda geodésica não-constante de $M$, quando $K \geq 0$; além disso, os subníveis de $\eta_{p_{0}}$ são compactos.

([19, Capítulo 4 - seção 4])

Considera-se a seguir quatro subvariedades do $\mathbb{R}^{3}$, todas com a métrica euclidiana induzida e exemplos de funções convexas definidas nestas superfícies.

EXEMPLO 2.44. A função altura $(x, y, z) \rightarrow z$ definida no parabolóide $z=x^{2}+y^{2}$, é um exemplo de função estritamente convexa, $C^{\infty}$, com um ponto de mínimo isolado em $(0,0,0)$, em uma variedade com curvatura positiva.

Escrevendo o parabolóide $S$ em equações paramétricas, obtem-se:

$$
\left\{\begin{array} { l } 
{ x = v \operatorname { c o s } u } \\
{ y = v \operatorname { s e n } u } \\
{ z = v ^ { 2 } }
\end{array} \quad ( u , v ) \in \left[0,2 \pi\left[\times \mathbb{R}^{+} .\right.\right.\right.
$$

As equações das geodésicas em $S$ são dadas por:

$$
\left\{\begin{array}{l}
u^{\prime \prime}+\frac{2}{v} u^{\prime} v^{\prime}=0 \\
v^{\prime \prime}-\frac{v}{1+4 v^{2}}\left(u^{\prime}\right)^{2}+\frac{4 v}{1+4 v^{2}}\left(v^{\prime}\right)^{2}=0 .
\end{array}\right.
$$

Sejam $F(x, y, z)=\frac{1}{2} z$ a função altura definida em $S$ e $\gamma$ uma geodésica não-constante em $S$, de modo que $\gamma(t)=(x(t), y(t), z(t))$ e $f(t)=F(\gamma(t))=\frac{1}{2} z(t)=\frac{1}{2}(v(t))^{2}$. Segue que:

$$
\begin{aligned}
& f^{\prime}=v v^{\prime} \\
& f^{\prime \prime}=\left(v^{\prime}\right)^{2}+v v^{\prime \prime} \\
& f^{\prime \prime}=\left(v^{\prime}\right)^{2}+v\left(\frac{v}{1+4 v^{2}}\left(u^{\prime}\right)^{2}-\frac{4 v}{1+4 v^{2}}\left(v^{\prime}\right)^{2}\right)=\frac{\left(v^{\prime}\right)^{2}+v^{2}\left(u^{\prime}\right)^{2}}{1+4 v^{2}}>0 .
\end{aligned}
$$


Portanto $F$ é estritamente convexa em $S$.

Observe que, como $F$ é estritamente convexa com ponto de mínimo isolado, vale a Proposição 2.41 e, de fato, o parabolóide é uma superfície contrátil. Mostra-se também que o parabolóide admite uma função de Busemann que é a função altura considerada acima.

EXEMPLO 2.45. Uma variedade simplesmente conexa e completa pode não admitir nenhuma função convexa não-constante, como é o caso da esfera $S^{2}$ já que é compacta (Corolário 2.37).

EXEMPLO 2.46. O cilindro $\left\{(x, y, z) \in \mathbb{R}^{3}: x^{2}+y^{2}=1\right\}$, uma superfície não-contrátil, admite funçôes convexas não-constantes, mas nenhuma estritamente convexa.

Uma geodésica normalizada no cilindro é dada por

$$
\left.\gamma(t)=\frac{1}{\sqrt{a^{2}+c^{2}}}(\cos (a t+b), \operatorname{sen}(a t+b)), c t+d\right), t \in \mathbb{R}
$$

Logo, as geodésicas são retas se $a=0$, círculos se $a \neq 0$ e $c=0$, ou hélices se $a \neq 0$ e $c \neq 0$. Destas, só as semi-retas são raios.

Dado $p=(1,0,0) \in S, \gamma(t)=(1,0, t), t \in[0,+\infty[$ é um raio que parte de $p$.

A função de Busemann relativa ao raio $\gamma, \eta_{\gamma}(x, y, z)=z$, é uma função convexa em $S$.

([19, página 120])

Como o cilindro possui geodésicas fechadas, pelo Corolário 2.37, $\eta_{\gamma}$ é constante ao longo delas, e portanto esta não é uma função convexa estrita.

Os raios partindo de $p=(1,0,0)$ são $(1,0, t)$ e $(1,0,-t), t \geq 0$. Segue que $h(x, y, z)=\sup _{\gamma} \eta_{\gamma}(x, y, z)=|z|$ também é convexa.

EXEMPLO 2.47. O hiperbolóide $\left\{(x, y, z) \in \mathbb{R}^{3} \backslash\{(x, y, 0)\}: x^{2}+y^{2}=\frac{1}{z^{2}}\right\}$ admite uma função convexa estrita $F(x, y, z)=\frac{1}{z}$, sem pontos críticos.

O hiperbolóide $S$ é dado pelas equações paramétricas:

$\left\{\begin{array}{l}x=v \cos u \\ y=v \operatorname{sen} u \\ z=\frac{1}{v}\end{array} \quad(u, v) \in\left[0,2 \pi\left[\times \mathbb{R}^{+} \backslash\{0\}\right.\right.\right.$.

As equações das geodésicas em $S$ são: $\left\{\begin{array}{l}u^{\prime \prime}+\frac{2}{v} u^{\prime} v^{\prime}=0 \\ v^{\prime \prime}-\frac{v^{5}}{1+v^{4}}\left(u^{\prime}\right)^{2}-\frac{2}{v+v^{5}}\left(v^{\prime}\right)^{2}=0 .\end{array}\right.$

Sejam $\gamma: \mathbb{R} \rightarrow S$ uma geodésica não-constante em $S$ e $f(t)=F(\gamma(t))=v(t)$. Tem-se que: $f^{\prime \prime}(t)=v^{\prime \prime}(t)=\frac{v^{5}}{1+v^{4}}\left(u^{\prime}\right)^{2}+\frac{2}{v+v^{5}}\left(v^{\prime}\right)^{2}>0$ para todo $t \in \mathbb{R}$. Portanto $F$ é estritamente convexa em $S$.

Note que $S$ não possui nenhuma geodésica fechada e daí a função $F$ é estritamente convexa; esta superfície é homotópica a um círculo, e logo é não-contrátil e de fato, $F$ não possui ponto crítico em $S$. Mesmo que uma variedade $S$, como é o 
caso deste hiperbolóide, seja difeomorfa ao cilindro que não admite nenhuma função estritamente convexa, a variedade $S$ pode admiti-la. Portanto, a existência de funções estritamente convexas não é invariante por difeomorfismos. É invariante por isometrias, que preservam as geodésicas.

Seja $(M, g)$ uma variedade Riemanniana completa, não-compacta e simplesmente conexa com um polo $p_{0}$ (Definição 1.57). Segue, pelo Lema 1.64, que cada $p \in M \backslash\left\{p_{0}\right\}$ é ligado a $p_{0}$ por uma única geodésica $\gamma_{p}:[0,1] \rightarrow M$ onde $\gamma_{p}(0)=p$ e $\gamma_{p}(1)=p_{0}$.

A curvatura radial relativa ao polo $p_{0}$ em $p$, denotada por $K_{r}(p)$, é definida como o máximo das curvaturas seccionais $K_{p}(\sigma)$ em todos os planos $\sigma$ contidos em $T_{p} M$ contendo a direção $\dot{\gamma}_{p}(1)$.

EXEMPLO 2.48. Se $(M, g)$ admite um polo $p_{0}$ tal que sua curvatura radial $K_{r} e ́$ não-positiva em todo ponto de $M$ então

$$
\begin{aligned}
d: M & \rightarrow \mathbb{R}^{+} \\
p & \rightarrow d(p)=\operatorname{dist}^{2}\left(p, p_{0}\right)
\end{aligned}
$$

é uma função estritamente convexa em $M$.

Prova. Será feita mostrando-se que Hess $d(p)$ é definido positivo para todo $p \in M$ ou seja, Hess $d(p)[v, v]>0$ para todo $v \in T_{p} M \backslash\{0\}$.

Sejam $p \in M$ e $v \in T_{p} M, v \neq 0$.

Considere $h:]-\epsilon, \epsilon[\rightarrow M$ a única geodésica passando por $p$ no instante $s=0 \mathrm{com}$ velocidade inicial $v=\dot{h}(0)$. Pelo Lema 1.23 ,

$$
\operatorname{Hess} d(p)[v, v]=\left.\frac{d^{2}}{d s^{2}} d(h(s))\right|_{s=0} .
$$

Denote $h(s)$ por $p_{s}$ e faça $\gamma_{s}=\gamma_{p_{s}}:[0,1] \rightarrow M$, a única geodésica em $M$ ligando $p_{s}$ a $p_{0}$. Ou seja, $\left.\gamma:\right]-\epsilon, \epsilon[\times[0,1] \rightarrow M$ é uma variação a um parâmetro da geodésica $\gamma_{p}$ tal que $\gamma_{s}(t)=\gamma_{p_{s}}(t)$ e $\gamma_{0}(s)=h(s)$; com um extremo fixo: $\gamma_{s}(1)=p_{0}$ para todo $s \in]-\epsilon, \epsilon\left[\right.$; e com campo variacional $v(t)$ ao longo de $\gamma_{p}$ dado por

$$
v(t)=\left.\frac{\partial}{\partial s} \gamma_{t}(s)\right|_{s=0}
$$

e tal que $v(0)=v$ e $v(1)=0$.

Como $\operatorname{dist}\left(p_{0}, p\right)=\int_{0}^{1}\left\|\dot{\gamma}_{p}(r)\right\| d r=\left\|\dot{\gamma}_{p}(t)\right\|=$ cte, $t \in[0,1]$,

tome $\left\|\dot{\gamma}_{p}(t)\right\|=\left\|\dot{\gamma}_{p}(T)\right\|$ para algum $T \in[0,1]$.

$\operatorname{Logo} \operatorname{dist}\left(p_{0}, p_{s}\right)=\int_{0}^{1}\left\|\dot{\gamma}_{s}(r)\right\| d r=\left\|\dot{\gamma}_{s}(T)\right\|=L(s)$. 
Assim, $d(h(s))=d\left(p_{s}\right)=L^{2}(s)=\left(\int_{0}^{1}\left\|\dot{\gamma}_{s}(T)\right\| d t\right)^{2}=\int_{0}^{1}\left\|\dot{\gamma}_{s}(t)\right\|^{2} d t \mathrm{e}$

$$
\begin{aligned}
\operatorname{Hess} d(p)[v, v] & =\left.\frac{d^{2}}{d s^{2}}\left(\int_{0}^{1} g\left(\dot{\gamma}_{s}(t), \dot{\gamma}_{s}(t)\right) d t\right)\right|_{s=0}=\left.\int_{0}^{1} \frac{d^{2}}{d s^{2}} g\left(\dot{\gamma}_{s}(t), \dot{\gamma}_{s}(t)\right)\right|_{s=0} d t \\
& =\left.\int_{0}^{1} 2 \frac{d}{d s} g\left(\frac{D}{d s} \dot{\gamma}_{s}(t), \dot{\gamma}_{s}(t)\right)\right|_{s=0} d t \\
& =2 \int_{0}^{1}\left[g\left(\frac{D}{d s} \frac{D}{d s} \dot{\gamma}_{s}(t), \dot{\gamma}_{s}(t)\right)+g\left(\frac{D}{d s} \dot{\gamma}_{s}(t), \frac{D}{d s} \dot{\gamma}_{s}(t)\right)\right]_{s=0} d t
\end{aligned}
$$

Como $\frac{D}{d s} \dot{\gamma}_{s}(t)=\frac{D}{d s} \frac{\partial}{\partial t} \gamma_{s}(t)$, pelo Lema da Simetria,

$$
\left.\frac{D}{d s} \dot{\gamma}_{s}(t)\right|_{s=0}=\left.\frac{D}{d t} \frac{\partial}{\partial s} \gamma_{t}(s)\right|_{s=0}=\frac{D}{d t} v(t)=\dot{v}(t) .
$$

Note que $\dot{v}(t) \neq 0$ pois, caso contrário, $v(t)$ seria um campo paralelo, e como $v(1)=0, v(t) \equiv 0$ o que é absurdo já que $v(0)=v \neq 0$.

E também, pela Proposição 1.8,

$$
\left.\frac{D}{d s} \frac{D}{d s} \dot{\gamma}_{s}(t)\right|_{s=0}=\left.\frac{D}{d s} \frac{D}{d t} \frac{\partial}{\partial s} \gamma_{t}(s)\right|_{s=0}=\frac{D}{d s} \frac{D}{d t} v(t)=\nabla_{v(t)} \nabla_{\dot{\gamma}(t)} v(t)
$$

onde $\nabla$ é a conexão Levi-Civita de $g$. Logo

$$
\text { Hess } d(p)[v, v]=2 \int_{0}^{1}\|\dot{v}(t)\|^{2}+g\left(\nabla_{v(t)} \nabla_{\dot{\gamma}(t)} v(t), \dot{\gamma}(t)\right) d t
$$

Por outro lado, pela definição de curvatura radial e usando $v$ e $\dot{\gamma}$ ao invés de $v(t)$ e $\dot{\gamma}(t)$ para simplificar a notação, tem-se por hipótese que:

$$
K_{p}(\sigma)=\frac{g(R(v, \dot{\gamma}) \dot{\gamma}, v)}{\|v \times \dot{\gamma}\|^{2}} \leq K_{r}(p) \leq 0
$$

onde $\sigma \subset T_{p} M$ é o plano gerado por $\{v, \dot{\gamma}\}$. Logo $g(R(v, \dot{\gamma}) v, \dot{\gamma}) \geq 0$.

Como $R(v, \dot{\gamma}) v=\nabla_{v} \nabla_{\dot{\gamma}} v-\nabla_{\dot{\gamma}} \nabla_{v} v-\nabla_{[v, \dot{\gamma}]} v$, pela simetria de $\nabla$ :

$$
[v(t), \dot{\gamma}(t)]=\left[\frac{D}{d s} \frac{\partial}{\partial t} \gamma(s, t)-\frac{D}{d t} \frac{\partial}{\partial s} \gamma(s, t)\right]_{s=0}=0
$$

e como $\nabla_{v} v=\nabla_{\dot{h}_{(0)}} \dot{h} \equiv 0$ obtem-se que $\nabla_{v} \nabla_{\dot{\gamma}} v=R(v, \dot{\gamma}) v$. Portanto

$$
\text { Hess } \begin{aligned}
d(p)[v, v] & =2 \int_{0}^{1}\|\dot{v}(t)\|^{2}+g(R(v(t), \dot{\gamma}(t)) v(t), \dot{\gamma}(t)) d t \\
& \geq 2 \int_{0}^{1}\|\dot{v}(t)\|^{2} d t>0 .
\end{aligned}
$$

Mostremos também que em $p_{0}$, Hess $d\left(p_{0}\right)$ é definido positivo.

Seja $\gamma:]-\delta, \delta\left[\rightarrow M\right.$ a única geodésica passando por $p_{0}$ no instante $t=0 \mathrm{com}$ velocidade $v \neq 0$. Então $d(\gamma(t))=\operatorname{dist}^{2}\left(p_{0}, \gamma(t)\right)=\|v\|^{2} t^{2}$. Pelo Lema 1.23,

$$
\operatorname{Hess} d\left(p_{0}\right)[v, v]=\left.\frac{d^{2}}{d t^{2}} d(\gamma(t))\right|_{t=0}=\left.\frac{d^{2}}{d t^{2}}\|v\|^{2} t^{2}\right|_{t=0}=2\|v\|^{2}>0 .
$$

Portanto, Hess $d(p)$ é definido positivo em todo $p \in M$ e pela Observação 2.33, $d$ é estritamente convexa em $M$. 
Observe que, neste caso, $p_{0}$ é um ponto de mínimo isolado de $d$ e logo, pela Proposição 2.41, $M$ é contrátil. E a Proposição 2.42 fornece um resultado análogo ao do Teorema de Hadamard-Cartan (1.58): M é difeomorfa a $\mathbb{R}^{m}$.

Em particular, sob as hipóteses do Teorema de Hadamard-Cartan ou seja, dada uma variedade Riemanniana simplesmente conexa, completa, com curvatura seccional não-positiva, para cada $p_{0} \in M$ fixado, a função

$$
d(p)=d i s t^{2}\left(p_{0}, p\right)
$$

é estritamente convexa em $M$. Pois, pelo Lema 1.59, todo ponto de $M$ é um polo.

Seja $X \in \mathfrak{X}(M)$ e considere a função real diferenciável $\left(C^{\infty}\right), F: M \rightarrow \mathbb{R}$

$$
F(p)=\frac{1}{2} g\left(X_{p}, X_{p}\right)
$$

a chamada função energia de $X$.

A análise da velocidade de variação de $F$ ao longo das trajetórias de $X$ assim como de seus pontos críticos permite conhecer as propriedades de um particular campo vetorial $X$. Como $g$ é definida positiva, os zeros de $F$ coincidem com os zeros de $X$, e estes zeros são pontos de mínimo global, e logo, pontos críticos de $F$. Por outro lado, existem muitos exemplos nos quais nem todos os pontos críticos de $F$ são os zeros de $X$.

Este tipo de problema, incluindo aqueles casos nos quais as energias são funções convexas, interessa diretamente à Física. Por exemplo, os pontos críticos da função Energia de um campo magnético $B$ (um campo solenoidal e irrotacional num aberto do $\mathbb{R}^{3}$ ) que não são zeros do campo são importantes na pesquisa sobre a Teoria da Estabilidade do Plasma e na Fusão Termonuclear controlada, porque estes pontos dão valores mínimos ou de sela para a energia com intensidade não-nula de $B$.

([19, Capítulo 5 - Teorema 5.11]).

Se $X \in \mathfrak{X}(M)$ é um campo irrotacional então satisfaz a propriedade de que: $g\left(\nabla_{Y} X, Z\right)=g\left(\nabla_{Z} X, Y\right)$ para todo $\operatorname{par} Y, Z \in \mathfrak{X}(M)$.

Considere $\alpha: I \subset \mathbb{R} \rightarrow M$ uma curva integral qualquer de $X$. Então a velocidade de variação da energia $F$ ao longo das órbitas de $X$, e em particular ao longo de $\alpha$, é dada por

$$
d F(Y)=\frac{1}{2} Y g(X, X)=g\left(\nabla_{Y} X, X\right)=g\left(\nabla_{X} X, Y\right) .
$$

Logo $\nabla F=\nabla_{X} X$. Se $\nabla_{X} X=0$ então $\alpha$ é uma geodésica de $(M, g)$.

Portanto, além de os zeros de $X$ serem pontos críticos de $F$, o conjunto dos pontos críticos de $F$ incluem as órbitas de $X$ que são geodésicas. 
Se $X \in \mathfrak{X}(M)$ é um campo de Killing (Definição 1.69) vale a propriedade:

$$
g\left(\nabla_{Y} X, Z\right)=-g\left(\nabla_{Z} X, Y\right)
$$

para todo $Y, Z \in \mathfrak{X}(M)$. Segue que:

$$
d F(Y)=\frac{1}{2} Y g(X, X)=g\left(\nabla_{Y} X, X\right)=-g\left(\nabla_{X} X, Y\right)
$$

e logo $d F(X)=0$ e também $\nabla F=-\nabla_{X} X$.

Portanto os zeros de $X$ são pontos críticos de $F$, aliás pontos de mínimo global, e além disso, os pontos críticos de $F$ são precisamente os pontos $p \in M$ tal que a curva integral de $X$, que passa por $p$, é uma geodésica.

Além disso, campos de Killing originam funções energia que são convexas com a condição de que a curvatura Killing associada a $M$ seja não-positiva.

A curvatura Killing associada em $p \in M, K(p)$, é definida como o máximo das curvaturas seccionais em todos os planos de $T_{p} M$ contendo a direção $X(p)$.

EXEMPLO 2.49. Se $X$ é um campo de Killing em $(M, g)$ cuja curvatura Killing associada a $M$ é não-positiva então a função energia de $X$,

$$
F(p)=\frac{1}{2} g\left(X_{p}, X_{p}\right)
$$

é convexa.

Se a curvatura Killing associada a todo $p$ em $M$ for estritamente negativa então F é estritamente convexa.

PROVA. É necessário mostrar que Hess $F(p)$ é semi-definido positivo ou seja, que Hess $F(p)[v, v] \geq 0$ para todo $v \in T_{p} M$ e em todo $p \in M$.

Sejam $p \in M$ e $v \in T_{p} M$. Considere $\left.\gamma:\right]-\epsilon, \epsilon[\rightarrow M$ a única geodésica em $M$ passando por $p$ em $t=0$ com velocidade inicial $v=\dot{\gamma}(0)$.

Pelo Lema 1.23,

$$
\text { Hess } \left.F(p)[v, v]=\frac{d^{2}}{d t^{2}} F(\gamma)(t)\right)\left.\right|_{t=0}=\left.\frac{1}{2} \frac{d^{2}}{d t^{2}} g(X(\gamma(t)), X(\gamma(t)))\right|_{t=0} .
$$

Denote por $X(t)$ o campo de Killing $X$ tomado ao longo de $\gamma$ e tal que $X(0)=X_{p}$. Então

$$
\begin{aligned}
& \frac{D}{d t} X(\gamma(t))=\nabla_{\dot{\gamma}(t)} X \mathrm{e} \\
& \text { Hess } F(p)[v, v]=\left.\frac{d}{d t} g\left(\nabla_{\dot{\gamma}(t)} X, X(t)\right)\right|_{t=0} .
\end{aligned}
$$

Como $X$ é um campo de Killing, vale a propriedade (2.9): $g\left(\nabla_{Y} X, Z\right)=-g\left(\nabla_{Z} X, Y\right)$ para todo par $Y, Z \in \mathfrak{X}(M)$. Assim,

$$
\operatorname{Hess} F(p)[v, v]=-\left.\frac{d}{d t} g\left(\nabla_{X(t)} X, \dot{\gamma}(t)\right)\right|_{t=0}=-\left.g\left(\nabla_{\dot{\gamma}(t)} \nabla_{X(t)} X, \dot{\gamma}(t)\right)\right|_{t=0}
$$

pois $\nabla_{\dot{\gamma}} \dot{\gamma} \equiv 0$ 
Por outro lado, como a curvatura Killing associada a $p$, por hipótese, é não-positiva:

$$
K_{p}(\sigma)=\frac{g\left(R\left(v, X_{p}\right) X_{p}, v\right)}{\left\|v \times X_{p}\right\|^{2}} \leq K(p) \leq 0
$$

onde $\sigma \subset T_{p} M$ é o plano gerado por $\left\{v, X_{p}\right\}$.

E simplificando a notação ou seja, tomando $X$ e $\dot{\gamma}$ ao invés de $X(t)$ e $\dot{\gamma}(t)$, a expressão do tensor curvatura de $g$ em $M$ é dada por:

$$
R(\dot{\gamma}, X) X=\nabla_{\dot{\gamma}} \nabla_{X} X-\nabla_{X} \nabla_{\dot{\gamma}} X-\nabla_{[\dot{\gamma}, X]} X \text {. Logo }
$$

Hess $F(p)[v, v]=-g(R(\dot{\gamma}, X) X, \dot{\gamma})-g\left(\nabla_{X} \nabla_{\dot{\gamma}} X, \dot{\gamma}\right)-\left.g\left(\nabla_{[\dot{\gamma}, X]} X, \dot{\gamma}\right)\right|_{t=0}$.

Por (2.9) e pela simetria da conexão, isto é, $[\dot{\gamma}, X]=\nabla_{\dot{\gamma}} X-\nabla_{X} \dot{\gamma}$, segue que:

$$
g\left(\nabla_{[\dot{\gamma}, X]} X, \dot{\gamma}\right)=-g\left(\nabla_{\dot{\gamma}} X,[\dot{\gamma}, X]\right)=-g\left(\nabla_{\dot{\gamma}} X, \nabla_{\dot{\gamma}} X\right)+g\left(\nabla_{\dot{\gamma}} X, \nabla_{X} \dot{\gamma}\right) .
$$

Ainda por (2.9) $g\left(\nabla_{\dot{\gamma}} X, \dot{\gamma}\right)=-g\left(\nabla_{\dot{\gamma}} X, \dot{\gamma}\right)$ e logo $g\left(\nabla_{\dot{\gamma}} X, \dot{\gamma}\right)=0$. Tomando-se a derivada covariante na direção do campo $X, X g\left(\nabla_{\dot{\gamma}} X, \dot{\gamma}\right)=0$ e então $g\left(\nabla_{X} \nabla_{\dot{\gamma}} X, \dot{\gamma}\right)=-g\left(\nabla_{\dot{\gamma}} X, \nabla_{X} \dot{\gamma}\right)$. Daí:

$$
\begin{aligned}
\text { Hess } F(p)[v, v]=-g(R(\dot{\gamma}, X) X, \dot{\gamma}) & -g\left(\nabla_{X} \nabla_{\dot{\gamma}} X, \dot{\gamma}\right) \\
& +g\left(\nabla_{X} \nabla_{\dot{\gamma}} X, \dot{\gamma}\right)+\left.g\left(\nabla_{\dot{\gamma}} X, \nabla_{\dot{\gamma}} X\right)\right|_{t=0} .
\end{aligned}
$$

Denotando $\nabla_{\dot{\gamma}(t)} X$ por $X^{\prime}(t)$, segue que:

$$
\text { Hess } F(p)[v, v]=-g\left(R\left(v, X_{p}\right) X_{p}, v\right)+\left\|X_{p}^{\prime}\right\|^{2} \geq 0 .
$$

Suponha agora que a curvatura Killing $K(p)$ é estritamente negativa. Afirma-se, então, que $X^{\prime}(t) \neq 0$ o que implica que Hess $F(p)[v, v]>0$ e portanto $F$ é estritamente convexa em $M$.

De fato, pois caso contrário, como

$$
K_{p}(\sigma)=\frac{g(R(\dot{\gamma}, X) X, \dot{\gamma})}{\|\dot{\gamma} \times X\|^{2}}
$$

e $X$ sendo de Killing, $X(t)$ é um campo de Jacobi ao longo da geodésica $\gamma$ e pela Definição 1.45, satisfaz: $X^{\prime \prime}=\nabla_{\dot{\gamma}} \nabla_{\dot{\gamma}} X=R(\dot{\gamma}, X) \dot{\gamma}=0$.

Então $K_{p}(\sigma) \equiv 0$ o que é absurdo pois $K_{p}(\sigma) \leq K(p)<0$.

No próximo exemplo, estabelece-se uma condição sobre a métrica $g$, conformemente plana, que garante a existência de uma função estritamente convexa em $(M, g)$, no caso particular em que $M$ é difeomorfa ao $\mathbb{R}^{m}$.

Seja $M$ difeomorfa a $\mathbb{R}^{m}$. Considere $H: \mathbb{R}^{m} \rightarrow M$ tal difeomorfismo. Seja $g$ conformemente plana em $M$ isto é, existe uma função diferenciável $\phi: \mathbb{R}^{m} \rightarrow \mathbb{R}^{+}$tal que $g=\phi \cdot g_{0}$ onde $g_{0}:=\langle$,$\rangle é a métrica euclidiana de \mathbb{R}^{m}$ ou 
seja, pela Definição 1.5 , se $H(x)=p$ e $v=\mathrm{d} H_{x}(y) \in T_{p} M$

(2.10) $g(v, v)_{p}=\phi(x)\langle y, y\rangle_{x}$

para todo $x, y \in \mathbb{R}^{m}$.

Denote por $\|$.$\| a norma e por \nabla$ o gradiente euclidianos isto é, $\mathrm{d} \phi_{x}(y)=\left\langle\nabla \phi_{x}, y\right\rangle$.

EXEMPLO 2.50. Se $\phi$ satisfaz $\phi(x)-\frac{3}{2}\|\nabla \phi(x)\| .\|x\|>0$ para todo $x \in \mathbb{R}^{m}$ então a função

$$
F(p)=F(H(x))=\frac{1}{2}\|x\|^{2}
$$

é estritamente convexa em $M$.

Prova. Seja $\gamma:[0,1] \rightarrow M$ uma geodésica normalizada em $M$, ié. $g(\dot{\gamma}, \dot{\gamma}) \equiv 1$; e $z:[0,1] \rightarrow \mathbb{R}^{m}$ a curva em $\mathbb{R}^{m}$ tal que $\gamma(s)=H(z(s))$. Logo $\dot{\gamma}=\mathrm{d} H_{z}(\dot{z})$ e segue, por (2.10), que

$$
g(\dot{\gamma}, \dot{\gamma})=\phi(z)\langle\dot{z}, \dot{z}\rangle
$$

Considere o funcional Energia $E[\gamma]=\frac{1}{2} \int_{0}^{1} g(\dot{\gamma}(t), \dot{\gamma}(t)) d t=\frac{1}{2} \int_{0}^{1} \phi(z)\langle\dot{z}, \dot{z}\rangle d t$. Diferenciando a ação integral $E_{\phi}(\gamma)$, e como $E_{\phi}^{\prime}(\gamma)=0$, pois $\gamma$ é um elemento crítico de $E$ (Corolário 1.42), obtem-se que:

$$
\begin{array}{ll} 
& \frac{\mathrm{d}}{\mathrm{d} s}(\phi(z)\langle\dot{z}, \dot{z}\rangle)=\langle\nabla \phi(z), \dot{z}\rangle\langle\dot{z}, \dot{z}\rangle+2 \phi(z)\langle\dot{z}, \ddot{z}\rangle=0 \\
\Longrightarrow \quad & -\langle\nabla \phi(z), \dot{z}\rangle\langle\dot{z}, \dot{z}\rangle-2 \phi(z)\langle\dot{z}, \ddot{z}\rangle=0 \\
\Longrightarrow & \langle\nabla \phi(z), \dot{z}\rangle\langle\dot{z}, \dot{z}\rangle-2\langle\nabla \phi(z), \dot{z}\rangle\langle\dot{z}, \dot{z}\rangle-2 \phi(z)\langle\ddot{z}, \dot{z}\rangle \\
= & 2\left\langle\frac{1}{2} \nabla \phi(z)\langle\dot{z}, \dot{z}\rangle, \dot{z}\right\rangle-2\langle\langle\nabla \phi(z), \dot{z}) \dot{z}, \dot{z}\rangle-2\langle\phi(z) \ddot{z}, \dot{z}\rangle \\
= & 2\left\langle\frac{1}{2} \nabla \phi(z)\langle\dot{z}, \dot{z}\rangle-\langle\nabla \phi(z), \dot{z}\rangle \dot{z}-\phi(z) \ddot{z}, \dot{z}\right\rangle=0 .
\end{array}
$$

Logo

(2.11) $\frac{1}{2} \nabla \phi(z)\langle\dot{z}, \dot{z}\rangle-\langle\nabla \phi(z), \dot{z}\rangle \dot{z}-\phi(z) \ddot{z}=0$

é a equação da geodésica $\gamma$ na métrica $g=\phi\langle$,$\rangle .$

Seja $\rho=F \circ \gamma:[0,1] \rightarrow \mathbb{R}$ e, como $\gamma(s)=(H \circ z)(s)$,

$\rho(s)=F(\gamma(s))=F(H(z(s)))=\frac{1}{2}\langle z(s), z(s)\rangle$

$\rho^{\prime}(s)=\langle z, \dot{z}\rangle$

$$
\rho^{\prime \prime}(s)=\langle\dot{z}, \dot{z}\rangle+\langle z, \ddot{z}\rangle=\|\dot{z}\|^{2}+\langle z, \ddot{z}\rangle .
$$

Como d $\phi_{z}(z)=\langle\nabla \phi(z), z\rangle>0$, pela desigualdade de Cauchy-Schwarz

$$
\begin{aligned}
& \langle z, \nabla \phi(z)\rangle>-\langle z, \nabla \phi(z)\rangle \geq-\|\nabla \phi(z)\|\|z\| \\
& \langle\nabla \phi(z), \dot{z}\rangle\langle z, \dot{z}\rangle \leq\|\nabla \phi(z)\|\|z\|\|\dot{z}\|^{2},
\end{aligned}
$$


e substituindo $\ddot{z}$ obtida em (2.11) em (2.12), segue que:

$$
\begin{aligned}
\rho^{\prime \prime}(s) & =\|\dot{z}\|^{2}+\frac{1}{2 \phi(z)}\langle z, \nabla \phi(z)\rangle\langle\dot{z}, \dot{z}\rangle-\frac{1}{\phi(z)}\langle z, \dot{z}\rangle\langle\nabla \phi(z), \dot{z}\rangle \\
& \geq\|\dot{z}\|^{2}-\frac{1}{2 \phi(z)}\|z\|\|\nabla \phi(z)\|\|\dot{z}\|^{2}-\frac{1}{\phi(z)}\|\nabla \phi(z)\|\|z\|\|\dot{z}\|^{2} \\
& =\|\dot{z}\|^{2}\left[1-\frac{3}{2 \phi(z)}\|z\|\|\nabla \phi(z)\|\right]
\end{aligned}
$$

Por hipótese

$$
\frac{3}{2 \phi(z(s))}\|z(s)\|\|\nabla \phi(z(s))\|<1,
$$

e $\log 0 \rho^{\prime \prime}(s)>0$. Portanto $\rho=F$ o $\gamma$ é estritamente convexa e segue que $F$ é estritamente convexa em $M$.

A seguir apresenta-se uma variedade em $\mathbb{R}^{2}$ que admite uma função estritamente convexa com ponto de mínimo e cuja curvatura muda de sinal.

EXEMPLO 2.51. Seja $S=\left(\mathbb{R}^{2}, \mathrm{~d} s^{2}\right)$, onde $\mathrm{d} s^{2}=\mathrm{d} r^{2}+f(r)^{2} \mathrm{~d} \theta^{2}$ em coordenadas polares $(r, \theta)$ e $f(r)$ é uma função diferenciável satisfazendo $\left\{\begin{array}{l}f(0)=0 \\ f^{\prime}(0)=1 \\ f(r)>0 \text { se } r>0 .\end{array}\right.$

Dados um ponto $p \in S$ tal que a distância $\operatorname{dist}(p, 0)=r$, e um vetor $v \in T_{p} S$ ortogonal ao raio $o p$, define-se a função distância $\rho(p)=\operatorname{dist}(p, 0)$. Então, a curvatura seccional $K_{p}$ é dada por $K_{p}=-\frac{f^{\prime \prime}(r)}{f(r)}$ e o hessiano Hess $\rho(p)[v, v]=\frac{f^{\prime}(r)}{f(r)}$. Assuma que $f$ é crescente e que $f^{\prime \prime}$ muda de sinal, então Hess $\rho(p)[v, v] \geq 0$. Portanto, a função $\rho$ é convexa com um ponto de mínimo isolado em 0 , não-constante ao longo de qualquer geodésica em $S$ com $K_{p}$ mudando de sinal.

Observe que dada uma função convexa não-constante $F: M \rightarrow \mathbb{R}$ em toda geodésica não-constante, então para toda função estritamente convexa $\psi: \mathbb{R} \rightarrow \mathbb{R}$ a composição $\psi \circ F$ é estritamente convexa em $M$.

Usando este método padrão, podemos obter uma função estritamente convexa $F$ com um mínimo em $S$. Para isto é suficiente tomar qualquer função real crescente e estritamente convexa $\psi$. Então $F=\psi \circ \rho$ é a função desejada.

([11, Exemplo 3.1]) 


\section{CAPíTULO 3}

\section{Sistemas Dinâmicos Conservativos em Variedades Riemannianas}

Equações diferenciais, sistemas dinâmicos ou campos de vetores definidos em uma variedade diferenciável aparecem naturalmente em muitas aplicações físicas, e além disso, do ponto de vista estritamente matemático, o lugar natural para se definir uma equação diferencial é uma variedade diferenciável.

Sejam, por exemplo, $n$ osciladores harmônicos dados pelas equações

$$
\ddot{x}_{i}=-x_{i} \quad i=1, \ldots, n \quad \text { ou } \quad\left\{\begin{array}{l}
\dot{x}_{i}=y_{i} \\
\dot{y}_{i}=-x_{i}
\end{array} \quad i=1, \ldots, n\right.
$$

de modo que temos um campo de vetores $X$ definido em $\mathbb{R}^{2 n}:\left(x_{1}, \ldots, x_{n}, y_{1}, \ldots, y_{n}\right)$.

Se quisermos estudar as trajetórias que possuem um valor fixo para a energia, digamos igual a 1, vemos que elas estão sobre a esfera $S^{2 n-1}:\left(x_{1}\right)^{2}+\cdots+\left(y_{n}\right)^{2}=1$ de modo que tal estudo equivale ao estudo do campo de vetores $X$ sobre $S^{2 n-1}$.

Se $a_{1}+\cdots+a_{n}=1$, os $a_{i}$ 's sendo positivos, então o toro $T^{n}$ definido por

$$
\left(x_{i}\right)^{2}+\left(y_{i}\right)^{2}=a_{i}, \quad i=1, \ldots, n
$$

é constituído por trajetórias de $X$ contidas em $S^{2 n-1}$ de modo que $X$ induz também um campo de vetores em $T^{n}$.

\section{Caracterização do sistema dinâmico (3.1) com condições de fronteira (3.2)}

Seja $(M, g)$ uma variedade Riemanniana completa e considere a seguinte equação diferencial definida em $M$ :

$$
\frac{D \dot{x}}{d t}=-\nabla V(x)
$$

onde $V: M \rightarrow \mathbb{R}$ é uma função de classe $C^{2}$. Assim, interpreta-se $M$ como o espaço das configurações de massas que se movem sob a ação de uma força conservativa e diz-se que a equação (3.1), conhecida como a Equação de Euler-Lagrange, é um sistema dinâmico conservativo em $M$ com potencial $-V$.

Fixe um ponto $p$ em $M$ e um número positivo $T$. As soluções de (3.1)

$$
x:[0, T] \rightarrow M
$$

são curvas cujas imagens $x([0, T])$ em $M$ representam as trajetórias do campo gradiente de $V$ partindo de $p$ no instante inicial e chegando na posição $x(T)=q$ após 
um tempo $T$. Assim, fixados p e q em $M$, nos interessa analisar aquelas soluções de (3.1) que satisfazem as chamadas condições de fronteira a dois valores:

$$
x(0)=p \text { e } x(T)=q .
$$

O sistema dinâmico (3.1) com as condições de fronteira (3.2) constituem o problema diferencial:

(I) $\left\{\begin{array}{l}\frac{D \dot{x}}{d t}=-\nabla V(x) \\ x:[0, T] \rightarrow M \text { tal que } x(0)=p \text { e } x(T)=q\end{array}\right.$

a ser estudado com o objetivo principal de estabelecer-se condições métricas e topológicas sobre $M$ e para o potencial $V$ que garantam a existência de soluções do sistema dinâmico (3.1) satisfazendo as condições de fronteira (3.2) e tais que o número destas soluções seja finito para quase todo par de pontos $p, q$ em $M$.

Lembremos da seguinte definição de E.D.O.:

Dado $X: U \subset M \rightarrow T M$ um campo vetorial $C^{1}$ em um aberto $U$ de $M$, considere $x:[a, b] \rightarrow M$ uma curva integral de $X$. Diz-se que a aplicação contínua $H: U \rightarrow \mathbb{R}$ é uma integral primeira de $X$ se $\mathrm{d} H_{x}=g(\nabla H, \dot{x})=g(\nabla H, X) \equiv 0$ isto é, $H$ é constante ao longo das trajetórias de $X$ e $H$ não é constante em nenhum conjunto aberto.

Denotando $\dot{x}=-y$, o sistema dinâmico (3.1), 'transferido' ao espaço de fases $T M=\{(x, y)\}$, é o sistema Hamiltoniano:

$\left\{\begin{array}{l}\dot{x}=-y \\ \frac{D y}{d t}=\nabla V .\end{array}\right.$

Assim, se a integral primeira $H=E_{c}+E_{p}$ representa a energia total do sistema, onde $E_{c}(x, y)=\frac{1}{2} g(y, y)$ é a energia cinética e $E_{p}(x, y)=V(x)$ é a energia potencial, então $H$, dita $o$ hamiltoniano do sistema, é constante ao longo de cada trajetória $x$.

LEMA 3.1. As soluções de (3.1) satisfazem a seguinte Lei de Conservação de Energia:

$$
\frac{1}{2} g(\dot{x}, \dot{x})+V(x) \equiv E_{x}=\text { cte para todo } t \in[0, T] .
$$

Prova. Seja $x:[0, T] \rightarrow M$ uma solução de (3.1). Como pela compatibilidade da métrica com a derivada covariante,

$$
\frac{d}{d t}\left(\frac{1}{2} g(\dot{x}, \dot{x})+V(x)\right)=g\left(\frac{D \dot{x}}{d t}, \dot{x}\right)+g(\nabla V(x), \dot{x})=g\left(\frac{D \dot{x}}{d t}+\nabla V(x), \dot{x}\right)
$$

e como $\frac{D \dot{x}}{d t}+\nabla V(x)=0$ para todo $t \in[0, T]$,

$$
\frac{d}{d t}\left(\frac{1}{2} g(\dot{x}, \dot{x})+V(x)\right) \equiv 0
$$

segue que $E_{x} \equiv$ cte. 
O sistema dinâmico (3.1) é autônomo isto é, o segundo membro da equação (3.1) independe do tempo, o que implica que o conjunto das soluções é invariante por reparametrizações que envolvam translações ou reversões do tempo. Por isso pudemos tomar $[0, T]$ como o domínio de definição das soluções de (3.1). Mas isto não ocorre para reparametrizações afins arbitrárias. Vejamos:

se $x:\left[t_{0}, t_{1}\right] \rightarrow M$ é solução de $\left\{\begin{array}{l}\frac{D \dot{x}}{d t}=-\nabla V(x) \\ x\left(t_{0}\right)=p \text { e } x\left(t_{1}\right)=q\end{array}\right.$

e tomarmos uma reparametrização de $x$ dada pelo isomorfismo $\phi(t)=s=k\left(t-t_{0}\right)$ tal que dado $T>0, \operatorname{com} k=\frac{T}{t_{1}-t_{0}} \neq 1$, a curva $y=x \circ \phi^{-1}:[0, T] \rightarrow M$ terá o mesmo traço de $x: y(s)=x\left(\phi^{-1}(s)\right)=x(t)$, e satisfará as condições de fronteira:

$$
\left\{\begin{array}{l}
y(0)=x\left(\phi^{-1}(0)\right)=x\left(t_{0}\right)=p \\
y(T)=x\left(\phi^{-1}(T)\right)=x\left(t_{1}\right)=q
\end{array}\right.
$$

mas a equação (3.1) não será satisfeita:

$$
\begin{aligned}
y(s) & =x\left(\phi^{-1}(s)\right) \\
\dot{y} & =\dot{x}(t) \frac{1}{k} \\
\frac{D \dot{y}}{d s} & =\frac{D \dot{x}}{d t}\left(\frac{1}{k}\right)^{2}=-\frac{1}{k^{2}} \nabla V(y) .
\end{aligned}
$$

e portanto $y$ não será solução do problema dado.

Vejamos um exemplo no espaço euclidiano $\mathbb{R}^{2}$ com a métrica canônica usual:

Sejam $X: \mathbb{R}^{2} \rightarrow \mathbb{R}^{2}$ um campo vetorial $C^{1}$ dado por $X(x, y)=(\dot{x}, \dot{y})$ e $H: \mathbb{R}^{2} \rightarrow \mathbb{R}$ contínua.

O sistema $\left\{\begin{array}{l}\dot{x}=\frac{\partial H}{\partial y} \\ \dot{y}=-\frac{\partial H}{\partial x}\end{array}\right.$

é hamiltoniano pois, para toda curva integral $\alpha$ de $X, \mathrm{~d} H_{\alpha}=\langle\nabla H, X\rangle \equiv 0$.

Daí decorre que as soluções de um sistema conservativo 'descem' as curvas de nível de $H$ pois, $\nabla H$ é normal às curvas de nível de $H$ e tem o sentido de seus valores crescentes.

Se $y=\dot{x}$ ou seja, $X(x, y)=(\dot{x}, \ddot{x})$ e $H(x, y)=\frac{1}{2} y^{2}+V(x), \operatorname{com} V: \mathbb{R}^{2} \rightarrow \mathbb{R}$ então o sistema hamiltoniano

$$
\left\{\begin{array}{l}
\dot{x}=y=\frac{\partial H}{\partial y} \\
\dot{y}=\ddot{x}=-\frac{\partial H}{\partial x}=-\nabla V(x)
\end{array}\right.
$$

equivale à equação diferencial $\ddot{x}=f(t, x, \dot{x})=-\nabla V(x)$.

O mais simples dos problemas do tipo (I) é dado por uma equação diferencial de segunda ordem $\ddot{x}=f(t, x, \dot{x})$, definida no plano euclidiano, tal que suas soluções $x:\left[t_{0}, t_{1}\right] \rightarrow \mathbb{R}^{2}$ passam por dois pontos fixos: $x\left(t_{0}\right)=\left(t_{0}, x_{0}\right)$ e $x\left(t_{1}\right)=\left(t_{1}, x_{1}\right)$. 
O problema é global porque os dois pontos podem não estar próximos um do outro e pode acontecer que nenhuma solução passe por êles. Na verdade, este é o problema diferencial global mais antigo, com condições de fronteira a dois valores, da teoria das equações diferenciais.

Uma questão pertinente que se coloca é a seguinte: Sob que condições há apenas um número finito de soluções que passam pelos pontos dados $\left(t_{0}, x_{0}\right)$ e $\left(t_{1}, x_{1}\right)$ ? Serão discutidas, na seção 3 do Capítulo 4, condições suficientes para que o número de soluções deste problema, definido em $\mathbb{R}^{n}$, seja finito. Um modelo interessante para este problema e que permite "visualizar" suas soluções é a chamada decomposição focal do plano introduzida por Peixoto[17] como segue:

Fixando o ponto inicial $\left(t_{0}, x_{0}\right)$ estuda-se como o número de soluções varia quando o extremo final $\left(t_{1}, x_{1}\right)$ varia no plano, e qual tipo de decomposição do plano é obtida considerando-se $\mathbb{R}^{2}=\bigcup_{i=0}^{\infty} \sigma_{i}$, onde cada $\sigma_{i}$ é o conjunto dos pontos $\left(t_{1}, x_{1}\right)$ para os quais há exatamente $i$ soluções. Como os $\sigma_{i}$ 's são disjuntos dois a dois, formam uma partição de $\mathbb{R}^{2}$.

Podemos reformular nosso resultado principal utilizando um modelo análogo ao da decomposição focal do plano em $M$.

DEFINIÇÃO 3.2. Sejam $\sigma_{i}=\{q \in M$ : existem exatamente $i$ soluções do problema $(I)\}_{i=0,1, \cdots,+\infty}$. Como $\bigcup_{i=0}^{\infty} \sigma_{i}=M$ e os $\sigma_{i}$ 's são disjuntos dois a dois, obtem-se uma partição de $M$, chamada de decomposição focal de $M$.

Assim, como quer-se estabelecer condições métricas e topológicas sobre $M$ que garantam a finitude do número de soluções do problema (I) equivalentemente vai-se estabelecer as condições tais que $\sigma_{\infty}$ tenha medida nula em $M$.

Considere um problema mais simples, para começar, também interessante para métricas não planas: o caso em que o potencial $V$ é nulo, ou constante, isto é, quando o sistema (3.1) se reduz à equação de geodésicas em $M$ ligando $p$ a $q$ :

(II) $\left\{\begin{array}{l}\frac{D \dot{x}}{d t} \equiv 0 \\ x:[0,1] \rightarrow M \text { tal que } x(0)=p \text { e } x(1)=q \text {. }\end{array}\right.$

Aqui foi possível tomar o intervalo $[0,1]$ para domínio de definição das soluções pois reparametrizações por isomorfismos preservam as geodésicas (Lema 1.29).

Se $M$ é completa, pelo Teorema de Hopf-Rinow (1.56), existe pelo menos uma geodésica ligando quaisquer dois pontos em $M$. E portanto (II) sempre terá solução.

Provavelmente, o primeiro resultado sobre finitude para esta situação é o Teorema de Hadamard-Cartan (1.58), que estabelece que se $M$ é simplesmente conexa, $(M, g)$ é completa, com curvatura seccional não-positiva, então entre quaisquer dois pontos de $M$ existe uma única geodésica. 
Na seção 2 do Capítulo 4 discute-se a finitude de soluções neste caso particular, provando-se que existe um número finito de geodésicas ligando dois pontos nãoconjugados em $M$ desde que $M$ admita uma função estritamente convexa com ponto de mínimo não-degenerado.

Este resultado também se baseia no fato de que as soluções de (II) são os pontos críticos do funcional Energia $E$

$$
E[\alpha]=\int_{a}^{b} \frac{1}{2} g(\dot{\alpha}(t), \dot{\alpha}(t)) d t .
$$

definido em $\Omega_{p, q}^{1}=\{\alpha:[a, b] \rightarrow M: \alpha(a)=p$ e $\alpha(b)=q\}$, o espaço de todas as curvas absolutamente contínuas em $M$ ligando $p$ a $q$ (Corolário 1.42).

Analogamente, considera-se $\Omega_{p, q}([0, T], M)$, o espaço de todas as curvas $\alpha:[0, T] \rightarrow M$ de classe $C^{1}$ em $M$ ligando $p$ a $q$, e logo, satisfazendo (3.2).

Dada $\alpha \in \Omega_{p, q}([0, T], M)$, o espaço tangente a $\Omega_{p, q}([0, T], M)$ em $\alpha$, um espaço de Hilbert de dimensão infinita, consiste de todos os campos vetoriais $C^{1}$ ao longo de $\alpha$ que se anulam em 0 e em $T$ ou seja:

$T_{\alpha} \Omega_{p, q}([0, T], M)=\left\{W:[0, T] \rightarrow T M, W(t) \in T_{\alpha(t)} M\right.$ e $\left.W(0)=W(T)=0\right\}$. Se $(M, g)$ é completa, $\Omega_{p, q}([0, T], M)$ também é uma variedade de Hilbert completa.

DEFINIÇÃ̇o 3.3. O funcional Lagrangiano $\mathcal{L}_{T}$ em $\Omega_{p, q}([\dot{0}, T], M)$ é a integral

$$
\mathcal{L}_{T}[\alpha]=\int_{0}^{T}\left[\frac{1}{2} g(\dot{\alpha}, \dot{\alpha})-V(\alpha)\right] d t .
$$

PROPOSIÇÃo 3.4. Primeira variação do funcional Lagrangiano

Sejam $\alpha \in \Omega_{p, q}([0, T], M)$ e $W \in T_{\alpha} \Omega_{p, q}([0, T], M)$.

A primeira variação do Lagrangiano $\mathcal{L}_{T}$ em $\alpha$ na direção $W$ é dada por:

$$
\mathrm{d} \mathcal{L}_{T}[\alpha] W=-\int_{0}^{T} g\left(\frac{D \dot{\alpha}}{d t}+\nabla V(\alpha), W(\alpha)\right) d t .
$$

PROVA. Seja $\left\{\alpha_{s}\right\}_{s \in]-\epsilon, \epsilon[}$ uma variação própria de $\alpha$ com campo variacional $W$.

$$
\begin{aligned}
\mathrm{d} \mathcal{L}_{T}[\alpha] W & =\left.\frac{d}{d s} \mathcal{L}_{T}\left[\alpha_{s}\right]\right|_{s=0}=\mathcal{L}_{T}^{\prime}(0)=\left.\frac{d}{d s} \int_{0}^{T}\left[\frac{1}{2} g\left(\dot{\alpha}_{s}(t), \dot{\alpha}_{s}(t)\right)-V\left(\alpha_{s}(t)\right)\right] d t\right|_{s=0} \\
& =\left.\int_{0}^{T} \frac{d}{d s}\left[\frac{1}{2} g\left(\frac{\partial \alpha_{s}}{\partial t}, \frac{\partial \alpha_{s}}{\partial t}\right)-V\left(\alpha_{s}\right)\right]\right|_{s=0} d t \\
& =\int_{0}^{T}\left[g\left(\frac{D}{d s} \frac{\partial \alpha_{s}}{\partial t}, \frac{\partial \alpha_{s}}{\partial t}\right)-g\left(\nabla V\left(\alpha_{s}\right), \frac{\partial \alpha_{t}}{\partial s}\right)\right]_{s=0} d t .
\end{aligned}
$$

Pelo Lema da Simetria (1.37) e pela compatibilidade da derivada covariante com a métrica (Corolário 1.16), obtem-se que:

$$
g\left(\frac{D}{d s} \frac{\partial \alpha_{s}}{\partial t}, \frac{\partial \alpha_{s}}{\partial t}\right)=g\left(\frac{D}{d t} \frac{\partial \alpha_{t}}{\partial s}, \frac{\partial \alpha_{s}}{\partial t}\right)=\frac{d}{d t} g\left(\frac{\partial \alpha_{t}}{\partial s}, \frac{\partial \alpha_{s}}{\partial t}\right)-g\left(\frac{\partial \alpha_{t}}{\partial s}, \frac{D}{d t} \frac{\partial \alpha_{s}}{\partial t}\right)
$$


e como, por definição (1.35), o campo variacional $W$ é um campo ao longo de $\alpha$ dado por $\left.\frac{\partial \alpha_{t}}{\partial s}\right|_{s=0}=W(t)$ segue que:

$$
\mathcal{L}_{T}^{\prime}(0)=\left.g(\dot{\alpha}, W(t))\right|_{0} ^{T}-\int_{0}^{T} g\left(\frac{D \dot{\alpha}}{d t}+\nabla V(\alpha), W(t)\right) d t .
$$

Portanto, como $W \in T_{\alpha} \Omega_{p, q}([0, T], M)$, tem-se o resultado desejado.

DEFINIÇão 3.5. Uma curva $x$ é crítica para $\mathcal{L}_{T}: \Omega_{p, q}([0, T], M) \rightarrow \mathbb{R} s e$,

$\mathrm{d} \mathcal{L}_{T}[x] W=0$, para todo $W \in T_{x} \Omega_{p, q}([0, T], M)$.

O próximo resultado é conhecido como o Princípio da Ação Estacionária de Hamilton: As soluções de um sistema conservativo são os pontos críticos, também chamados de pontos estacionários, da ação integral $\mathcal{L}_{T}$ ([18, seção A2.15]).

PROPOSIÇÃo 3.6. $x$ é solução do problema (I) se e somente se $x$ é ponto estacionário da ação integral $\mathcal{L}_{T}$.

Prova. Por definição (3.5), $x$ é ponto estacionário de $\mathcal{L}_{T}$ se

$$
\mathrm{d} \mathcal{L}_{T}[x] W=-\int_{0}^{T} g\left(\frac{D \dot{x}}{d t}+\nabla V(x), W(t)\right) d t=0
$$

para todo $W \in T_{x} \Omega_{p, q}([0, T], M)$. Segue pelo Corolário 1.38 que

$$
\frac{D \dot{x}}{d t}+\nabla V(x)=0
$$

para quase todo $t \in[0, T]$ e portanto $x$ é solução da equação (3.1),satisfazendo (3.2). A recíproca é imediata.

PROPOSIÇÃo 3.7. Segunda Variação do funcional Lagrangiano Dado um ponto crítico $x \in \Omega_{p, q}([0, T], M)$ de $\mathcal{L}_{T}$, a segunda variação $\mathrm{d}^{2} \mathcal{L}_{T}[x]$ de $\mathcal{L}_{T}$ em $x$ é a seguinte forma bilinear simétrica limitada ${ }^{1}$

(3.3) $\mathrm{d}^{2} \mathcal{L}_{T}[x](W, Z)=\int_{0}^{T}\left[g\left(W^{\prime}, Z^{\prime}\right)+g(R(\dot{x}, W) \dot{x}, Z)-\operatorname{Hess} V(x)(W, Z)\right] d t$ definida no espaço de Hilbert $T_{x} \Omega_{p, q}([0, T], M)$.

Prova. Sejam $W \in T_{\alpha} \Omega_{p, q}([0, T], M)$ e $\left\{x_{s}\right\}_{s \in]-\epsilon, \epsilon[}$ uma variação própria de $x$ com campo variacional $W$. A forma quadrática $\mathrm{d}^{2} \mathcal{L}_{T}[x](W, W)$ é dada por $\left.\frac{d^{2}}{d s^{2}} \mathcal{L}_{T}\left[x_{s}\right]\right|_{s=0}=\left.\frac{d}{d s} \mathcal{L}_{T}^{\prime}(s)\right|_{s=0}=\mathcal{L}_{T}^{\prime \prime}(0)$.

Pela Proposição 3.4, tem-se: $\mathrm{d} \mathcal{L}_{T}[x] W=-\int_{0}^{T} g\left(\frac{D \dot{x}}{d t}+\nabla V(x), W(t)\right) d t$ e logo

$$
\mathcal{L}_{T}^{\prime}(s)=-\int_{0}^{T}\left[g\left(\frac{D}{d t} \frac{\partial x_{s}}{\partial t}, \frac{\partial x_{t}}{\partial s}\right)+g\left(\nabla V\left(x_{t}\right), \frac{\partial x_{t}}{\partial s}\right)\right] d t
$$

Como, pela definição (1.22) do hessiano de $V$ em $x_{i}(s)$ :

\footnotetext{
${ }^{1}$ Dada uma forma bilinear $B$ em um espaço vetorial $V$, $B$ é limitada em $V$ se $\sup _{\|v\|,\|w\| \leq 1}|B(v, w)|<+\infty$, para todo $v, w \in V$.
} 


$$
\begin{aligned}
g\left(\frac{D}{d s} \nabla V\left(x_{t}\right), \frac{\partial x_{t}}{\partial s}\right) & =g\left(\nabla_{\dot{x}_{t}(s)} \nabla V\left(x_{t}(s)\right), \frac{\partial x_{t}}{\partial s}(s)\right)=\operatorname{Hess} V\left(x_{t}(s)\right)\left[\frac{\partial x_{t}}{\partial s}(s), \frac{\partial x_{t}}{\partial s}(s)\right], \\
\mathcal{L}_{T}^{\prime \prime}(s)= & -\int_{0}^{T} g\left(\frac{D}{d s} \frac{D}{d t} \frac{\partial x_{s}}{\partial t}, \frac{\partial x_{t}}{\partial s}\right)+g\left(\frac{D}{d t} \frac{\partial x_{s}}{\partial t}, \frac{D}{d s} \frac{\partial x_{t}}{\partial s}\right) \\
+ & \operatorname{Hess} V\left(x_{t}\right)\left(\frac{\partial x_{t}}{\partial s}, \frac{\partial x_{t}}{\partial s}\right)+g\left(\nabla V\left(x_{t}\right), \frac{D}{d s} \frac{\partial x_{t}}{\partial s}\right) d t .
\end{aligned}
$$

Como $x$ é ponto crítico de $\mathcal{L}_{T}$, pela Proposição 3.6, $x$ é solução de (3.1) e logo, em $s=0, \frac{D}{d t} \frac{\partial x}{\partial t}+\nabla V(x) \equiv 0$.

Tomando a expressão do tensor curvatura de $g$ em $M$, obtem-se que:

$$
-g\left(\frac{D}{d s} \frac{D}{d t} \frac{\partial x_{s}}{\partial t}, \frac{\partial x_{t}}{\partial s}\right)=g\left(R\left(\frac{\partial x_{s}}{\partial t}, \frac{\partial x_{t}}{\partial s}\right) \frac{\partial x_{s}}{\partial t}, \frac{\partial x_{t}}{\partial s}\right)-g\left(\frac{D}{d t} \frac{D}{d s} \frac{\partial x_{s}}{\partial t}, \frac{\partial x_{t}}{\partial s}\right) .
$$

Como

$$
g\left(\frac{D}{d t} \frac{D}{d s} \frac{\partial x_{s}}{\partial t}, \frac{\partial x_{t}}{\partial s}\right)=\frac{d}{d t} g\left(\frac{D}{d s} \frac{\partial x_{s}}{\partial t}, \frac{\partial x_{t}}{\partial s}\right)-g\left(\frac{D}{d s} \frac{\partial x_{s}}{\partial t}, \frac{D}{d t} \frac{\partial x_{t}}{\partial s}\right)
$$

e usando o Lema da Simetria, $\left.\frac{D}{d s} \frac{\partial x_{s}}{\partial t}\right|_{s=0}=\left.\frac{D}{d t} \frac{\partial x_{t}}{\partial s}\right|_{s=0}=W^{\prime}$, segue que:

$$
\mathcal{L}_{T}^{\prime \prime}(0)=\int_{0}^{T}\left[g(R(\dot{x}, W) \dot{x}, W)+g\left(W^{\prime}, W^{\prime}\right)-\operatorname{Hess} V(x)(W, W)\right] d t
$$

pois, como $W(0)=W(T)=0,\left.g\left(W^{\prime}, W\right)\right|_{0} ^{T}=0$.

Usando a identidade de polarização obtem-se a expressão desejada a partir da forma quadrática $\mathrm{d}^{2} \mathcal{L}_{T}[x](W, W)=\mathcal{L}_{T}^{\prime \prime}(0)$ determinada acima.

\section{Conjugação ao longo das soluções de (3.1) e V-Campos de Jacobi}

Os pontos que são conjugados em relação a um sistema dinâmico tem um papel crucial na teoria. Em linhas gerais, dois pontos $p$ e $q$ são conjugados relativamente a um sistema dinâmico, como veremos adiante, se existe uma variação $\left\{x_{s}\right\}_{s \in]-\epsilon, \epsilon[}$ de soluções do sistema satisfazendo as condições de fronteira $x_{s}(0)=p$ e $x_{s}(T)=q$.

DEFINIÇão 3.8. Um ponto crítico $x$ para o funcional $\mathcal{L}_{T}$ em $\Omega_{p, q}([0, T], M) e ́$ dito não-degenerado se a segunda variação $\mathrm{d}^{2} \mathcal{L}_{T}[x]$ é uma forma bilinear fortemente não-degenerada em $T_{x} \Omega_{p, q}([0, T], M)$, isto é, quando o operador auto-adjunto sobre $T_{x} \Omega_{p, q}([0, T], M)$ que representa $\mathrm{d}^{2} \mathcal{L}_{T}[x]$ é um isomorfismo, e portanto, invertível.

DEFINIÇÃo 3.9. Um ponto $q \in M$ é dito conjugado a $p$ sobre $[0, T]$ relativamente a (3.1) se existe um ponto crítico degenerado $x$ de $\mathcal{L}_{T}$ em $\Omega_{p, q}([0, T], M)$.

Como a orientação no tempo é invariante, a noção de conjugação sobre $[0, T]$ é simétrica em $p$ e $q$. Assim, se $q$ é conjugado a $p$ sobre $[0, T]$ relativamente a (3.1) pode-se dizer que $p$ e $q$ são pontos conjugados. A noção de conjugação também pode ser dada em termos de V-Campos de Jacobi, definidos a seguir. 
DEFINIÇÃO 3.10. Um V-Campo de Jacobi ao longo de uma solução $x$ de (3.1) é um campo vetorial $J$ ao longo de $x$ que satisfaz a equação:

$$
J^{\prime \prime}-R(\dot{x}, J) \dot{x}+\text { hess } V(x)_{J}=0
$$

onde hess $V(x)_{J}$ é o operador hessiano de $V$ em $x$ na direção $J$.

LEMA 3.11. Um campo vetorial $J, C^{2}$, ao longo de uma solução $x:[0, T] \rightarrow M$ de (3.1) é um V-campo de Jacobi se e somente se existe uma variação de $x$ através de uma família de soluções de (3.1) sobre $[0, T],\left\{x_{s}\right\}_{s \in]-\epsilon, \epsilon[}$ com campo variacional $J$ isto é,

$$
\left.\frac{\partial}{\partial s} x_{s}(t)\right|_{s=0}=J(t), t \in[0, T] .
$$

Prova. Seja $x:[0, T] \rightarrow M$ uma solução de (3.1).

Considere $J$ um V-campo de Jacobi ao longo de $x,\left.J\right|_{x(t)}=J(t)$.

Seja $\epsilon>0$ suficientemente pequeno para garantir as hipóteses de regularidade assumidas a seguir. Defina $\gamma:]-\epsilon, \epsilon[\rightarrow M$ uma curva regular em $M$ satisfazendo: $\left\{\begin{array}{l}\gamma(0)=x(0) \\ \dot{\gamma}(0)=J(0)\end{array}\right.$

e $v:]-\epsilon, \epsilon\left[\rightarrow T M\right.$ um campo vetorial regular ao longo de $\gamma$ isto é, $\left.v\right|_{\gamma(s)}=v(s)$ satisfazendo: $\left\{\begin{array}{l}v(0)=\dot{x}(0) \\ v^{\prime}(0)=J^{\prime}(0)\end{array}\right.$

Para cada $s \in]-\epsilon, \epsilon$ [ denote por $t \rightarrow x_{s}(t)$ a solução maximal de (3.1) em $M$ satisfazendo $\left\{\begin{array}{l}x_{s}(0)=x_{0}(s)=\gamma(s) \\ \left.\frac{\partial}{\partial t} x_{s}(t)\right|_{t=0}=\dot{x}_{s}(0)=v(s) .\end{array}\right.$

Logo $\mathrm{x}_{0} \equiv \mathrm{x}$ sobre $[0, T]$ e, por continuidade, para $\epsilon$ suficientemente pequeno, $x_{s}$ está definida em $[0, T]$.

Seja $\tilde{J}$ o V-campo de Jacobi ao longo de $x$ que corresponde ao campo variacional de $\left\{x_{s}\right\}$. Então:

$\tilde{J}(0)=\left.\frac{d}{d s} x_{0}(s)\right|_{s=0}=\dot{\gamma}(0)=J(0)$

$\tilde{J}^{\prime}(0)=\left.\frac{d}{d t}\left(\frac{\partial}{\partial s} x_{t}(s)\right)_{s=0}\right|_{t=0}$. Pelo Lema da Simetria:

$\tilde{J}^{\prime}(0)=\left.\frac{d}{d s}\left(\frac{\partial}{\partial t} x_{s}(t)\right)_{t=0}\right|_{s=0}=\left.\frac{d}{d s} v(s)\right|_{s=0}=v^{\prime}(0)=J^{\prime}(0)$.

Como estas condições iniciais determinam unicamente uma solução da equação (3.4), conclui-se que $\tilde{J} \equiv J$. Portanto $J$ é o campo variacional de $\left\{x_{s}\right\}$.

A recíproca é provada através da linearização de (3.1) ou seja, como $\left\{x_{s}\right\}_{s \in]-\epsilon, \epsilon \mid}$ satisfazem a equação (3.1)

$$
\frac{D}{d t} \dot{x_{s}}=-\nabla V\left(x_{s}\right)
$$

para todo $t \in[0, T]$, segue que:

$$
\frac{D}{d s} \frac{D}{d t} \frac{\partial x_{s}}{\partial t}=-\frac{D}{d s} \nabla V\left(x_{s}\right) .
$$


Considerando o operador hessiano de $V$ em $x_{s}(t): \frac{D}{d s} \nabla V\left(x_{s}\right)=$ hess $V\left(x_{s}\right)_{\dot{x}_{s}}$, e como, pelo Lema da Simetria, $\left[\frac{\partial x_{s}}{\partial s}, \frac{\partial x_{s}}{\partial t}\right]=0 \mathrm{e}$

$$
\frac{D}{d s} \frac{\partial x_{s}}{\partial t}=\frac{D}{d t} \frac{\partial x_{t}}{\partial s}=\dot{x_{t}}(s)
$$

pela expressão do tensor curvatura de $g$, obtendo-se que:

$$
\frac{D}{d t} \frac{D}{d s} \frac{\partial x_{s}}{\partial t}-R\left(\frac{\partial x_{s}}{\partial t}, \frac{\partial x_{t}}{\partial s}\right) \frac{\partial x_{s}}{\partial t}=-\operatorname{hess} V\left(x_{t}\right)_{\dot{x}_{t}(s)} .
$$

Como $J$ é o campo variacional da variação dada, $J(t)=\left.\dot{x}_{t}(s)\right|_{s=0}$, para $s=0$ :

$$
J^{\prime \prime}-R(\dot{x}, J) \dot{x}=- \text { hess } V(x)_{J} .
$$

Portanto $J$ é um V-Campo de Jacobi ao longo de $x$.

Assim, por definição, V-Campos de Jacobi $J$ ao longo de uma solução $x$ são campos vetoriais variacionais que correspondem a variações de $x$ através de soluções $x_{s}:[0, T] \rightarrow M$ de (3.1). Ou seja: considerando a aplicação que a cada $\left.s \in\right]-\epsilon, \epsilon[$ associa $x_{s}, C^{2},\left\{x_{s}\right\}_{s \in]-\epsilon, \epsilon[}$ uma família de soluções de (3.1) tal que $x_{0}=x \mathrm{e}$ $\left.\frac{\partial}{\partial s} x_{s}(t)\right|_{s=0}=J(t) \in T_{x(t)} M$. Se $x \in \Omega_{p, q}([0, T], M)$ isto é, se $x$ também é solução de (3.2), então $J \in T_{x} \Omega_{p, q}([0, T], M)$.

$\mathrm{d}^{2} \mathcal{L}_{T}[x]$ é denotado por $I$ e o espaço nulo de $\mathrm{d}^{2} \mathcal{L}_{T}[x]$, Nuc $(I)$, é constituído pelos V-Campos de Jacobi. Compare com o caso particular da segunda variação do funcional Energia (Proposição 1.51).

LEMA 3.12. Seja $x \in \Omega_{p, q}([0, T], M)$ um ponto crítico de $\mathcal{L}_{T}$. Um campo vetorial $J \in T_{x} \Omega_{p, q}([0, T], M)$ é um V-campo de Jacobi ao longo de $x$ se e somente se $J$ está no núcleo de $I$ isto é, se e somente se $I[J, W]=0$ para todo $W \in T_{x} \Omega_{p, q}([0, T], M)$.

Prova. Observe inicialmente que, por um argumento padrão de análise (bootstrap) não é difícil mostrar que $J$ é de classe $C^{2}$ e como

$$
\begin{aligned}
& \frac{d}{d t} g\left(J^{\prime}, W\right)=g\left(J^{\prime \prime}, W\right)+g\left(J^{\prime}, W^{\prime}\right) \mathrm{e} \\
& \int_{0}^{1}\left[g\left(J^{\prime \prime}, W\right)+g\left(J^{\prime}, W^{\prime}\right)\right] d t=\left.g\left(J^{\prime}, W\right)\right|_{0} ^{T}=0,
\end{aligned}
$$

segue, pela Proposição 3.7, que

$$
I[J, W]=\int_{0}^{T}\left[g\left(R(\dot{x}, J) \dot{x}-J^{\prime \prime}, W\right)-\operatorname{Hess} V(x)(J, W)\right] d t .
$$

e considerando o operador hessiano hess $V(x)_{J}$ obtem-se que:

$$
I[J, W]=\int_{0}^{T} g\left(R(\dot{x}, J) \dot{x}-J^{\prime \prime}-\operatorname{hess} V(x)_{J}, W\right) d t .
$$


Se $J$ é um V-campo de Jacobi, satisfaz a equação (3.4), e segue imediatamente que $I[J, W]=0$ para todo $W \in T_{x} \Omega_{p, q}([0, T], M)$.

Suponha que $J \in \operatorname{Nuc}(I)$. Pelo Corolário 1.38, $J$ é um campo vetorial ao longo de $x$ que satisfaz a equação $J^{\prime \prime}-R(\dot{x}, J) \dot{x}+$ hess $V(x)_{J}=0$ e portanto, por definição (3.10) $J$ é um V-Campo de Jacobi.

LEMA 3.13. Para a forma bilinear $I$, as condições de fraca e fortemente nãodegeneração são equivalentes, isto é: I é fortemente não-degenerada se e somente $\operatorname{se} \operatorname{Nuc}(I)=\{0\}$.

Prova. Inicialmente, lembremos que, se $E_{1}$ e $E_{2}$ são espaços de Hilbert então a aplicação linear limitada $T: E_{1} \rightarrow E_{2}$ é Fredholm se a dimensão e a codimensão de $\operatorname{Nuc}(T)$ são finitas. Este resultado é uma consequência imediata do Teorema do gráfico fechado tal que $T\left(E_{1}\right)$ é fechado em $E_{2}$ ([14]). Então, afirma-se que:

a forma bilinear $I$ é representada no espaço de Hilbert $T_{x} \Omega_{p, q}([0, T], M)$ por um operador Fredholm de índice zero, denotado por $A_{x}: T_{x} \Omega_{p, q}([0, T], M) \rightarrow \mathbb{R}$.

De fato, como o $\operatorname{Nuc}\left(A_{x}\right)=\operatorname{Nuc}(I)$ é constituído por V-campos de Jacobi, atuando em $M$, e que satisfazem uma equação diferencial de segunda ordem com condição inicial $J(0)=0, \operatorname{dim} \operatorname{Nuc}(I)$ é no máximo igual a $m$.

A forma bilinear

$$
(v, w) \rightarrow \int_{0}^{T} g\left(v^{\prime}, w^{\prime}\right) d t
$$

é representada por um isomorfismo positivo de $T_{x} \Omega_{p, q}([0, T], M)$, que é um operador Fredholm de índice zero. Além disso, a forma bilinear

$$
(v, w) \rightarrow \int_{0}^{T}[g(R(\dot{x}, v) \dot{x}, w)-\operatorname{Hess} V(x)(v, w)] d t
$$

é contínua na $C^{0}$ topologia e por inclusão compacta de $C^{0} \mathrm{em} H^{1}$ segue que ela é representada por um operador auto-adjunto compacto em $T_{x} \Omega_{p, q}([0, T], M)$. Como perturbações compactas de operadores Fredholm são ainda operadores Fredholm com o mesmo índice, segue que $I$ é representado por um operador Fredholm de índice zero. Logo $I$ é fortemente não-degenerado se e somente se $\operatorname{Nuc}(I)=\{0\}$.

Estabelece-se a seguir a relação entre pontos conjugados e V-Campos de Jacobi.

PROPOSIÇÃo 3.14. Um ponto $q \in M$ é conjugado a p sobre $[0, T]$ relativamente a (3.1) se e somente se existe uma solução $x:[0, T] \rightarrow M$ de (3.1) satisfazendo (3.2) e existe um V-campo de Jacobi $J$ ao longo de $x$, não-nulo, tal que $J(0)=J(T)=0$.

Prova. Se existe um V-Campo de Jacobi $J$, não-nulo, ao longo de $x$, solução de (I), tal que $J(0)=J(T)=0$ então $J \in T_{x} \Omega_{p, q}([0, T], M)$. Pelo Lema 3.12, $J$ 
pertence ao núcleo de $I$ e, como $\operatorname{Nuc}(I) \neq\{0\}$, pelo Lema 3.13 segue que $x$ é um ponto crítico degenerado de $\mathcal{L}_{T}$. Portanto, por definição (3.9), $p$ e $q$ são conjugados sobre $[0, T]$ relativamente a $(3.1)$.

Suponha agora que $p$ e $q$ são conjugados sobre $[0, T]$ relativamente a (3.1). Por definição existe um ponto crítico degenerado $x$ de $\mathcal{L}_{T}$ em $\Omega_{p, q}([0, T], M)$. Pelo Lema 3.13 existe $J \in T_{x} \Omega_{p, q}([0, T], M)$, não nulo, tal que $J \in N u c(I)$ e portanto $J$ é um V-Campo de Jacobi ao longo de $x$ (Lema 3.12) com $J(0)=J(T)=0$.

Pode-se agora começar a estabelecer condições suficientes em $(M, g)$ e sobre $V$ que garantam que $\sigma_{\infty}$ tenha medida nula isto é, tal que para quase toda escolha de $q$ o número de trajetórias do sistema dinâmico (3.1) que começam em $p$ e terminam em $q$, após um tempo $T$ fixado, seja finito. Tendo em vista este objetivo, afirma-se que: $o$ conjunto dos pontos que são conjugados relativamente a um dado sistema dinâmico tem medida nula em $M$. A prova desta afirmação, dada a seguir, utiliza uma técnica análoga ao caso da conjugação através de geodésicas onde os pontos conjugados a um dado ponto $p$ em $M$ são caracterizados como os valores críticos da aplicação exponencial em $p$ (Proposição 1.50). A conclusão é obtida pelo Teorema de Sard:

Seja $f: M \rightarrow N$ uma aplicação $C^{1}$ entre variedades diferenciáveis de mesma dimensão $m$. Seja $S$ o conjunto dos pontos $x \in M$ nos quais a diferencial $\mathrm{d} f_{x}$ não é um isomorfismo. Então $f(S)$ tem medida nula em $N$. [10, Capítulo 6-seção 2].

Proposição 3.15. Seja $p \in M$ fixo. O conjunto dos pontos $q \in M$ que são conjugados a $p$ sobre $[0, T]$ relativamente a (3.1) tem medida nula em $M$.

Prova. Seja $\mathcal{F}_{p}: A \subset T_{p} M \rightarrow M$ uma aplicação $C^{1}$ definida em uma vizinhança aberta $A$ de 0 em $T_{p} M$, dada por:

$$
\mathcal{F}_{p}(v)=x(T)
$$

onde $x:[0, T] \rightarrow M$ é a única solução de (3.1) tal que $x(0)=p$ e $\dot{x}(0)=v$.

A diferenciabilidade de $\mathcal{F}_{p}$ é padrão: como no caso da aplicação exponencial, $\mathcal{F}_{p}$ é obtida a partir do fluxo de algum campo vetorial $C^{1}$ em $T M$, que é $C^{1}$ também.

Então, afirma-se que: $\mathrm{O}$ vetor $v \in A$ é um ponto crítico de $\mathcal{F}_{p}$, isto é, a diferencial $\left(\mathrm{d} \mathcal{F}_{p}\right)_{v}: T_{p} M \rightarrow T_{\mathcal{F}_{p}(v)} M$ é não-sobrejetora, se e somente se $x(T)=\mathcal{F}_{p}(v)$ é conjugado a $p$ ao longo de $x$.

De fato, pelo Lema 3.11, se $J$ é o campo variacional de uma variação $\left\{x_{s}\right\}_{s \in]-\epsilon, \epsilon}$, $J(t)=\left.\frac{\partial}{\partial s} x_{s}\right|_{s=0}, t \in[0, T] ; v(0)=\left.v \mathrm{e} \frac{\partial}{\partial s} v(s)\right|_{s=0}=v^{\prime}(0)=J^{\prime}(0)=w \neq 0$.

Como $\left\{x_{s}\right\}$ é uma família de soluções de (3.1) sobre $[0, T]$ satisfazendo (3.2), tem-se que $\gamma(s)=x_{s}(0)=p$ para todo $\left.s \in\right]-\epsilon, \epsilon[$ e então $\dot{\gamma}(0)=J(0)=0$. 
Tomando a diferencial de $\mathcal{F}_{p}(v(s))=x_{s}(T)$ em $s=0$ :

obtem-se que

$$
\left.\left(\mathrm{d} \mathcal{F}_{p}\right)_{v(s)}\left(\frac{\partial}{\partial s} v(s)\right)\right|_{s=0}=\left.\frac{\partial}{\partial s} x_{s}(T)\right|_{s=0}
$$

$$
\left(\mathrm{d} \mathcal{F}_{p}\right)_{v}(w)=J(T) .
$$

Então $J$ é o único V-campo de Jacobi ao longo de $x$ tal que $J(0)=0$ e $J^{\prime}(0)=w$. Como $\left(\mathrm{d} \mathcal{F}_{p}\right)_{v}$ é não-sobrejetora se e somente se $\operatorname{Nuc}\left(\left(\mathrm{d} \mathcal{F}_{p}\right)_{v}\right) \neq\{0\}$; se existe $w$, não nulo, $w \in \operatorname{Nuc}\left(\left(\mathrm{d} \mathcal{F}_{p}\right)_{v}\right)$ então $\left(\mathrm{d} \mathcal{F}_{p}\right)_{v}(w)=J(T)=0$ e portanto, pela Proposição 3.14, $\mathcal{F}_{p}(v)=x(T)=q$ é conjugado a $p$.

Assim, os pontos $q$ que são conjugados a $p$ são os valores críticos de $\mathcal{F}_{p}$ e portanto, pelo Teorema de Sard, este conjunto tem medida nula em $M$.

2.1. Multiplicidade dos instantes conjugados relativamente a (3.1). A noção de instantes conjugados ao longo de uma solução $x$ de (3.1), desenvolvida a seguir, está relacionada ao índice Morse do funcional $\mathcal{L}_{T}$ no ponto crítico $x$ (Definição 1.44).

DEFINIÇÃo 3.16. Seja $x:[0, T] \rightarrow M$ uma solução de (3.1). Um instante $\left.\left.t_{0} \in\right] 0, T\right]$ em $M$ é dito conjugado ao longo de $x$ se existe um V-Campo de Jacobi, não-nulo, ao longo de $x \operatorname{com} J(0)=J\left(t_{0}\right)=0$.

A multiplicidade mul $\left(t_{0}\right)$ do instante conjugado $t_{0}$ é definida como a dimensão do espaço gerado pelos V-Campos de Jacobi ao longo de x tais que $J(0)=J\left(t_{0}\right)=0$, isto é, é igual ao número máximo de tais campos l.i.; é claro que mul( $\left.t_{0}\right) \leq m$.

Observe que, pela Proposição 3.14, um instante $\left.t_{0} \in\right] 0, T$ ] é conjugado ao longo de $x$ se e somente se o ponto $x\left(t_{0}\right)$ é conjugado a $x(0)$ sobre $\left[0, t_{0}\right]$ relativamente a (3.1), com $\left.x\right|_{\left[0, t_{0}\right]}$ sendo o correspondente ponto crítico degenerado de $\mathcal{L}_{t_{0}}$ em $\Omega_{x(0), x\left(t_{0}\right)}\left(\left[0, t_{0}\right], M\right)$.

Dada uma solução $x:[0, T] \rightarrow M$ de (3.1),

$\mathrm{J}_{x}$ denota o espaço vetorial m-dimensional de todos os V-Campos de Jacobi, $J$, ao longo de $x$ satisfazendo $J(0)=0$ e $\phi_{t_{0}}: \mathbb{J}_{x} \rightarrow T_{x\left(t_{0}\right)} M$ é a aplicação de avaliação de $J \rightarrow J\left(t_{0}\right)$, para todo $\left.\left.t_{0} \in\right] 0, T\right]$.

LEMA 3.17. $t_{0}$ é um instante conjugado ao longo de $x$ se e somente se $\phi_{t_{0}}$ nãoé um isomorfismo.

Além disso, se t 0 é um instante conjugado ao longo de $x$ então sua multiplicidade é igual à codimensão da imagem $\operatorname{Im}\left(\phi_{t_{0}}\right)$ em $T_{x\left(t_{0}\right)} M$.

PROVA. Se $t_{0}$ é instante conjugado ao longo de $x$, por definição (3.16), existe $J$, um V-campo de Jacobi, não-nulo, ao longo de $x:[0, T] \rightarrow M$, uma solução de (3.1), 
$\operatorname{com} J(0)=J\left(t_{0}\right)=0$. Logo $\phi_{t_{0}}(J)=J\left(t_{0}\right)=0$ e segue que $J \in$ Nuc $\left(\phi_{t_{0}}\right)$. Portanto $\phi_{t_{0}}$ não é um isomorfismo.

Suponha agora que $\phi_{t_{0}}: \mathbb{J}_{x} \rightarrow T_{x\left(t_{0}\right)} M$ não é um isomorfismo, e logo não é injetora. Então existe $J \in \mathbb{J}_{x}$, um V-campo de Jacobi ao longo de $x \operatorname{com} J(0)=0$, tal que $\phi_{t_{0}}(J)=J\left(t_{0}\right)=0$. Portanto, por definição, $t_{0}$ é um instante conjugado ao longo de $x$.

Como a multiplicidade do instante conjugado $t_{0}, \operatorname{mul}\left(t_{0}\right)$, é igual à dimensão do espaço gerado pelos V-campos de Jacobi ao longo de $x$ tais que $J(0)=J\left(t_{0}\right)=0$, segue imeditamente que a dimensão do Núcleo de $\phi_{t_{0}}$ é igual a mul $\left(t_{0}\right)$.

LEMA 3.18. Se $J_{1}, J_{2}$ são $V$-campos de Jacobi ao longo de $x$ então a quantidade $g\left(J_{1}^{\prime}, J_{2}\right)-g\left(J_{1}, J_{2}^{\prime}\right)$ é constante.

Em particular, se $J_{1}, J_{2} \in \mathbb{J}_{x}$ então $g\left(J_{1}^{\prime}, J_{2}\right)-g\left(J_{1}, J_{2}^{\prime}\right) \equiv 0$.

Esta propriedade é dita uma lei de conservação satisfeita por V-campos de Jacobi.

Prova. Tem-se que: $\frac{d}{d t} g\left(J_{1}^{\prime}, J_{2}\right)-\frac{d}{d t} g\left(J_{1}, J_{2}^{\prime}\right)=g\left(J_{1}^{\prime \prime}, J_{2}\right)-g\left(J_{1}, J_{2}^{\prime \prime}\right)$. Como $J_{1}$ e $J_{2}$ são V-campos de Jacobi, por definição (3.10), satisfazem a equação (3.4): $J^{\prime \prime}-R(\dot{x}, J) \dot{x}+$ hess $V(x)_{J}=0$ obtem-se que:

$$
\begin{aligned}
\frac{d}{d t}\left(g\left(J_{1}^{\prime}, J_{2}\right)-g\left(J_{1}, J_{2}^{\prime}\right)\right) & =g\left(R\left(\dot{x}, J_{1}\right) \dot{x}, J_{2}\right)-g\left(\operatorname{Hess} V\left(J_{1}\right), J_{2}\right) \\
& -g\left(R\left(\dot{x}, J_{2}\right) \dot{x}, J_{1}\right)+g\left(\operatorname{Hess} V\left(J_{2}\right), J_{1}\right)=0
\end{aligned}
$$

pois o hessiano de V é uma forma bilinear simétrica e pela propriedade (3-d) do tensor curvatura de $g$, dada na Definição 1.19 .

Se $J_{1}, J_{2} \in \mathbb{J}_{x}, J_{1}(0)=J_{2}(0)=0$ e então $g\left(J_{1}^{\prime}(0), J_{2}(0)\right)-g\left(J_{1}(0), J_{2}^{\prime}(0)\right)=0$. Portanto $g\left(J_{1}^{\prime}, J_{2}\right)-g\left(J_{1}, J_{2}^{\prime}\right) \equiv$ constante $=0$.

Usando o lema anterior é possível adaptar a prova clássica de que o conjunto dos pontos conjugados ao longo de uma geodésica é discreto (Corolário 1.54), para provar a seguinte:

Proposição 3.19. Seja $x:[0, T] \rightarrow M$ uma solução de (3.1). Então o conjunto dos instantes conjugados ao longo de $x$ é finito.

Prova. Seja $\left.\left.t_{0} \in\right] 0, T\right]$ um instante conjugado ao longo de $x$.

Para provar a Proposição, vai-se mostrar que não existem outros instantes conjugados em uma vizinhança de $t_{0}$.

Com este objetivo, tome $k=\operatorname{mul}\left(t_{0}\right)$ e escolha $J_{1}, \cdots, J_{m}$ uma base do espaço $\mathbb{J}_{x}$. Pelo Lema 3.17, os vetores $J_{k+1}\left(t_{0}\right), \cdots, J_{m}\left(t_{0}\right)$ formam uma base da $\operatorname{Im}\left(\phi_{t_{0}}\right)$.

Além disso, as derivadas $J_{1}^{\prime}\left(t_{0}\right), \cdots, J_{k}^{\prime}\left(t_{0}\right)$ formam uma base do espaço ortogonal $\operatorname{Im}\left(\phi_{t_{0}}\right)^{\perp}$. De fato, estes $k$ vetores em $T_{x(t)} M$ são l.i., porque os vetores $J_{i}(t)$ 
e $J_{i}^{\prime}(t) i=1, \cdots, k$ pertencem ao conjunto-solução do sistema linear equivalente à equação diferencial de segunda ordem (3.4), aqui, com valor inicial $J_{i}\left(t_{0}\right)=0$.

E pelo Lema 3.18, de fato pertencem ao espaço ortogonal $\operatorname{Im}\left(\phi_{t_{0}}\right)^{\perp}$ pois $g\left(J_{i}^{\prime}\left(t_{0}\right), J_{j}\left(t_{0}\right)\right)=g\left(J_{i}\left(t_{0}\right), J_{j}^{\prime}\left(t_{0}\right)\right)=0$ para todo $i=1, \cdots, k$ e $j=k+1, \cdots, m$. Como $T_{x\left(t_{0}\right)} M=\operatorname{Im}\left(\phi_{t_{0}}\right) \oplus \operatorname{Im}\left(\phi_{t_{0}}\right)^{\perp}$,

conclui-se que a família $\left(J_{1}^{\prime}\left(t_{0}\right), \cdots, J_{k}^{\prime}\left(t_{0}\right), J_{k+1}\left(t_{0}\right), \cdots, J_{m}\left(t_{0}\right)\right)$ é uma base de $T_{x\left(t_{0}\right)} M$.

Defina agora a família $\left(\tilde{J}_{i}\right)_{i=1, \cdots, m}$ de campos vetoriais $C^{2}$ ao longo de $x$ fazendo:

$\tilde{J}_{i}=J_{i}$ para $i=k+1, \cdots, m \mathrm{e}$

$\tilde{J}_{i}(t)=\left\{\begin{array}{l}\frac{J_{i}\left(t_{0}\right)-J_{i}(0)}{t-t_{0}}=\frac{J_{i}\left(t_{0}\right)}{t-t_{0}} \text { para } t \neq t_{0} \\ J_{i}^{\prime}\left(t_{0}\right) \text { se } t=t_{0}\end{array}\right.$

Um instante $t \neq t_{0}$ em ]0,T] é conjugado ao longo de $x$ se e somente se $h(t)=\operatorname{det}\left(\tilde{J}_{1}(t), \cdots, \tilde{J}_{m}(t)\right)=0$ pois para $t \neq t_{0}$ o anulamento deste determinante é equivalente ao anulamento $\operatorname{do} \operatorname{det}\left(J_{1}(t), \cdots, J_{m}(t)\right)=0$.

Agora, como os $\tilde{J}_{i}\left(t_{0}\right)$ 's são 1.i., pois formam uma base de $T_{x\left(t_{0}\right)} M$, tem-se que $h\left(t_{0}\right) \neq 0$ e por continuidade numa vizinhança de $t_{0}, h(t) \neq 0$.

Isto implica que o conjunto dos instantes conjugados a $t_{0}$, ao longo de $x$, é discreto em $[0, T]$ ou seja, em um conjunto compacto, e portanto é finito.

A seguir enuncia-se o Teorema do Índice, o principal resultado sobre o índice Morse de $\mathcal{L}_{T}$. Compare com o Teorema 1.53 aplicado ao caso do funcional Energia.

Proposição 3.20. Seja $x:[0, T] \rightarrow M$ uma solução de (3.1) satisfazendo (3.2) isto é, x é um ponto crítico do funcional $\mathcal{L}_{T}$ em $\Omega_{p, q}([0, T], M)$. Então, o indice Morse de $\mathcal{L}_{T}$ em $x$ é finito, e é igual ao número de instantes conjugados ao longo de $x$ em ]0,T] contado com multiplicidade. 


\section{CAPíTULO 4}

\section{Finitude de Soluções de Sistemas Dinâmicos}

\section{Finitude de soluções do sistema dinâmico (3.1), satisfazendo (3.2)}

Nesta seção prova-se a existência e a finitude do número de soluções de (3.1) ligando dois pontos $p$ e $q$ não-conjugados em uma variedade Riemanniana $(M, g)$ completa que admite uma função estritamente convexa.

Começa-se com um lema sobre a convergência $C^{2}$ de sequências de curvas cuja aceleração covariante vai a zero uniformemente. Este resultado é trivial quando a métrica é plana, isto é, quando a aceleração covariante coincide com a segunda derivada. Para o caso geral, o argumento é mais delicado, devido ao fato de que, para o uso de coordenadas locais (no caso de ser necessário tomar-se mais de um aberto de $M$ ) precisa-se primeiro provar a convergência uniforme da sequência.

LEMA 4.1. Sejam $(M, g)$ uma variedade Riemanniana completa e $y_{n}:[a, b] \rightarrow M$ uma sequência de aplicações $C^{2}$ tal que $y_{n}^{\prime}(a)$ converge para $v_{0}$ em TM $e\left\|\frac{D}{d t} y_{n}^{\prime}\right\|$ converge a 0 , uniformemente em $[a, b]$. Então $y_{n}$ converge, na topologia $C^{2}$, para a geodésica $y:[a, b] \rightarrow M \operatorname{com} y^{\prime}(a)=v_{0}$.

PROVA. Mostra-se inicialmente que:

(i) $\left\|y_{n}^{\prime}\right\|$ é uniformemente limitada em $[a, b]$.

Seja $u_{n}=\left\|\frac{D}{d t} y_{n}^{\prime}\right\|$. Denote por $\delta_{n}=g\left(y_{n}^{\prime}, y_{n}^{\prime}\right)$. Segue que: $\delta_{n}^{\prime}=2 g\left(\frac{D}{d t} y_{n}^{\prime}, y_{n}^{\prime}\right)$.

Pela desigualdade de Cauchy-Schwarz:

$$
\left|\delta_{n}^{\prime}\right| \leq 2\left\|\frac{D}{d t} y_{n}^{\prime}\right\|\left\|y_{n}^{\prime}\right\|=2 u_{n} \sqrt{\delta_{n}} \leq 2 u_{n}\left(\delta_{n}+1\right) .
$$

Define-se $\left\|u_{n}\right\|_{\infty}=\sup _{t \in[a, b]}\left\|u_{n}(t)\right\|$ e como, por hipótese, $u_{n}$ converge uniformemente a 0 quando $n \rightarrow \infty$, existe $k=2 \sup _{n}\left\|u_{n}\right\|_{\infty}$ tal que $\left\|u_{n}\right\| \leq \frac{k}{2}$ para todo $n \in \mathbb{N}$.

O Lema de Gronwall, usado no próximo passo, fornece o seguinte resultado:

Sejam $f, g:[a, b] \rightarrow \mathbb{R}$ contínuas e $K \in \mathbb{R}$ tais que $f(t) \leq K+\int_{a}^{t} g(s) f(s) d s$ e $g(t) \geq 0$ para todo $t \in[a, b]$. Então

$$
f(t) \leq K \mathrm{e}^{\int_{a}^{t} g(s) d s}
$$

para todo $t \in[a, b]$.

Como $\int_{a}^{t} \delta_{n}^{\prime} d s \leq \int_{a}^{t}\left|\delta_{n}^{\prime}\right| d s$ e $\left|\delta_{n}^{\prime}\right| \leq 2 u_{n}\left(\delta_{n}+1\right)$ 
$\Longrightarrow \int_{a}^{t} \delta_{n}^{\prime} d s \leq \int_{a}^{t} 2 u_{n}\left(\delta_{n}+1\right) d s$

$\Longrightarrow \delta_{n}(t)-\delta_{n}(a) \leq \int_{a}^{t} 2 u_{n} \delta_{n} d s+\int_{a}^{t} 2 u_{n} d s$, e logo

$$
\delta_{n}(t) \leq \int_{a}^{t} 2 u_{n} \delta_{n} d s+\left(\delta_{n}(a)+\int_{a}^{t} 2 u_{n} d s\right)
$$

estão satisfeitas as hipóteses do Lema de Grownwall, pois com $K=\sup _{n}\left(\delta_{n}(a)+\int_{a}^{t} 2 u_{n} d s\right) \geq 0$, tem-se que

$$
\delta_{n}(t) \leq \int_{a}^{t} 2 u_{n} \delta_{n} d s+K \text { e } u_{n}=\left\|\frac{D}{d t} y_{n}^{\prime}\right\| \geq 0 .
$$

Segue, então que:

$$
\left|\delta_{n}(t)\right| \leq K \mathrm{e}^{2 \int_{a}^{t} u_{n} d s} .
$$

Como $2 \int_{a}^{t} u_{n} d s \leq 2 \int_{a}^{b} u_{n} d s \leq 2 \int_{a}^{b}\left\|u_{n}\right\| d s \leq k(b-a)$,

$$
\left|\delta_{n}(t)\right| \leq K \mathrm{e}^{k(b-a)}
$$

e portanto $\left\|y_{n}^{\prime}\right\|=\sqrt{\delta_{n}}$ é uniformemente limitado em $[a, b]$.

Seja $C=\sup _{n}\left\|y_{n}^{\prime}\right\|_{\infty}$.

(ii) Seja $y:[a, b] \rightarrow M$ a geodésica com $y^{\prime}(a)=v_{0}$.

Seja $r>0$ um raio normal para todos os pontos da imagem de $y$ (Teorema 1.33).

Escolha $\epsilon>0$ tal que $\epsilon<\frac{r}{c}$ e $\epsilon<\frac{r}{\left\|v_{0}\right\|}$.

Afirma-se que:

Se $y_{n}^{\prime}\left(t_{0}\right)$ converge para $y^{\prime}\left(t_{0}\right)$ para algum $t_{0} \in[a, b]$

então $y_{n}$ converge para $y$, no intervalo $\left[t_{0}-\epsilon, t_{0}+\epsilon\right] \cap[a, b]$.

A prova do Lema segue diretamente desta afirmação, pois para $t_{0}=a$, ocorre por hipótese que $y_{n}^{\prime}(a)$ converge para $v_{0}=y^{\prime}(a)$ em $T M$.

Observe que, como $y_{n}^{\prime}\left(t_{0}\right) \in T_{y_{n}\left(t_{0}\right)}^{\prime} M$, tem-se que $\left(y_{n}\left(t_{0}\right), y_{n}^{\prime}\left(t_{0}\right)\right) \in T M$ converge para $\left(y\left(t_{0}\right), y^{\prime}\left(t_{0}\right)\right) \in T M$.

Prova da afirmação:

Seja $U=\mathcal{B}\left(y\left(t_{0}\right), r\right)$.

Como dist $\left(y\left(t_{0}\right), y(t)\right)=\left\|y^{\prime}(a)\right\|\left|t-t_{0}\right| \leq\left\|v_{0}\right\| . \epsilon<r$, segue que $\left.y\right|_{\left[t_{0}-\epsilon, t_{0}+\epsilon\right] \cap[a, b]}$ tem imagem em $U$; e como, por hipótese, $y_{n}\left(t_{0}\right)$ converge para $y\left(t_{0}\right),\left.y_{n}\right|_{\left[t_{0}-\epsilon, t_{0}+\epsilon\right] \cap[a, b]}$ tem imagem em $U$ para todo $n$ suficientemente grande. 
Seja $w_{n}=\frac{D}{d t} y_{n}^{\prime}$ e usando coordenadas locais em $y_{n}\left(t_{0}\right) \in U$, para $n$ suficientemente grande, pela Proposição 1.12 , a $k$-ésima coordenada de $w_{n}\left(t_{0}\right)$ é dada por:

$$
\left(w_{n}\right)^{k}=\left(y_{n}^{\prime \prime}\right)^{k}+\sum_{i, j=1}^{m}\left(y_{n}^{\prime}\right)^{i}\left(y_{n}^{\prime}\right)^{j} \Gamma_{i j}^{k}\left(y_{n}\right) .
$$

Como, por hipótese, $w_{n}$ tende uniformemente a $0, y_{n}^{\prime}\left(t_{0}\right)$ converge para $y^{\prime}\left(t_{0}\right) \mathrm{e}$ $y_{n}\left(t_{0}\right)$ converge para $y\left(t_{0}\right)$ em $\left[t_{0}-\epsilon, t_{0}+\epsilon\right] \cap[a, b]$, resultados conhecidos sobre a dependência contínua entre a solução e os dados iniciais de um problema de Cauchy garantem que $y_{n}$ tende para a geodésica $y$ no intervalo dado, na topologia $C^{2}$. Isto prova a afirmação e em consequência o lema.

Este resultado permite assumir que se as soluções de um sistema dinâmico conservativo estiverem contidas em um subconjunto compacto de $M$ e com energia divergindo, então estas soluções tendem a geodésicas isto é, as trajetórias de partículas com energia cinética muito grande tendem a ser "linhas retas", sem fazer caso de forças gravitacionais. Nesta situação, pode-se utilizar as técnicas-padrão de convexidade em $M$ para mostrar um resultado inicial, ainda parcial, sobre a finitude de soluções de (3.1) ligando dois pontos $p$ e $q$ não-conjugados, cujas imagens estão contidas em um subconjunto compacto de $M$. Mas para tanto serão necessários os seguintes fatos básicos da teoria dos pontos críticos.

DEFINIÇão 4.2. Uma função $f$, de classe $C^{2}$, é dita uma função de Morse se todos os seus pontos críticos são não-degenerados.

Como no caso de funções diferenciáveis, $C^{\infty}$, em uma variedade diferenciável de dimensão finita, se $f$ é uma função de Morse em uma variedade Hilbertiana $(X, h)$ então cada ponto crítico de $f$ é isolado.

Lembre que foi provado na Proposição 3.14 que toda solução $x$ de (3.1) satisfazendo (3.2) é um ponto crítico não-degenerado para o funcional $\mathcal{L}_{T}$ e logo, que $\mathcal{L}_{T}$ é uma função de Morse em $\Omega_{p, q}([0, T], M)$, desde que $p$ e q sejam pontos nãoconjugados sobre $[0, T]$ relativamente a (3.1).

A principal condição técnica para desenvolver a Teoria de Morse em uma variedade Hilbertiana de dimensão infinita é a condição de compacidade de PalaisSmale: Diz-se que $f: X \rightarrow \mathbb{R}$, um funcional $C^{1}$ definido em uma variedade Hilbertiana $(X, h)$, satisfaz a condição P-S ao nível $c \in \mathbb{R}$ se toda sequência $\left(x_{n}\right)$ em $X$ tal que

$$
\left\{\begin{array}{l}
\lim _{n \rightarrow \infty} f\left(x_{n}\right)=c \\
\lim _{n \rightarrow \infty} h\left(\nabla f\left(x_{n}\right), \nabla f\left(x_{n}\right)\right)=0
\end{array}\right.
$$

tem uma subsequência convergente em $X$, onde $\nabla f(x)$ denota o gradiente de $f$ em $x \in X$, que é um operador linear limitado no espaço de Hilbert $T_{x} X$, definido por 
$\mathrm{d} f_{x}=h(\nabla f(x),$.$) . A sequência \left(x_{n}\right)$ satisfazendo (4.1) é chamada uma sequência Palais-Smale para $f$ em $X$ ao nivel $c$.

Além disso, pelo importante Teorema da Teoria de Morse:

$S e(X, h)$ é completa, o funcional $f$ é limitado inferiormente em $X$ e satisfaz a condição $P$-S então existe um ponto de mínimo de $f$.

([14, seção 9.1])

Observe que, se $\left(x_{n}\right)$ é qualquer sequência em $X$ para a qual $\left|f\left(x_{n}\right)\right|$ é limitado e $\left\|\mathrm{d} f_{x_{n}}\right\| \rightarrow 0$ então $\left(x_{n}\right)$ tem uma subsequência convergente, $x_{n_{k}} \rightarrow p$. Por continuidade $\left\|\mathrm{d} f_{x_{n}}\right\|=0$ e logo, p é um ponto crítico de $f$. Tem-se também que, se $X$ é compacta, independentemente da métrica em $X$, todas as três condições do teorema acima são automaticamente satisfeitas.

DEFINIÇão 4.3. Diz-se que uma aplicação, $C^{2}, V: M \rightarrow \mathbb{R}$ tem crescimento sub-quadrático se existem constantes $C_{1}, C_{2} \in \mathbb{R}^{+}$e $\left.\alpha \in\right] 0,2[$ tal que

$$
V(x) \leq C_{1}+C_{2} \cdot \operatorname{dist}\left(x, x_{0}\right)^{\alpha}
$$

para todo $x \in M$ e para algum $x_{0}$ fixado em $M$.

Assumindo-se que o potencial $V$ tem crescimento sub-quadrático, obtem-se o seguinte resultado bem conhecido:

LEMA 4.4. Se V tem crescimento sub-quadrático, o funcional Lagrangiano $\mathcal{L}_{T}$ é limitado inferiormente em $\Omega_{p, q}([0, T], M)$ e satisfaz a condição $P-S$.

Pelo Lema 4.4, se $V$ é limitado em $M$ então as condições do Teorema acima são satisfeitas pelo funcional Lagrangiano $\mathcal{L}_{T}$ em $\Omega_{p, q}([0, T], M)$ completa. Portanto existe um ponto crítico de $\mathcal{L}_{T}$, solução de (3.1) e (3.2). Garante-se assim, sob estas condições, a existência de soluções de (3.1) satisfazendo (3.2).

PROPOSIÇÃo 4.5. Seja $(M, g)$ uma variedade Riemanniana completa. Seja $V: M \rightarrow \mathbb{R}$ uma aplicação de classe $C^{2}$ e assuma que $M$ admite uma função estritamente convexa $F: M \rightarrow \mathbb{R}$. Considere o sistema dinâmico (3.1), com condições iniciais (3.2) e suponha que $p$ e q são não-conjugados sobre $[0, T]$ relativamente a (3.1). Então, para todo subconjunto compacto $K \subset M \operatorname{com} p, q \in K$, há apenas um número finito de soluções com imagem em $K$.

Prova. Seja $K \subset M$ um subconjunto compacto de $M$, fixado. Assuma, por negação, que $\left(x_{k}\right)_{k=1, \cdots, \infty}$ é uma sequência de soluções distintas duas a duas de (3.1) e (3.2), cujas imagens permanecem em $K$.

A prova da Proposição será obtida mostrando que, então, $p$ e $q$ devem ser conjugados relativamente a (3.1) sobre $[0, T]$. 
Agora, suponha, por absurdo, que $p$ e $q$ são não-conjugados. Então, deve ocorrer

$$
\lim _{k \rightarrow \infty} \mathcal{L}_{T}\left[x_{k}\right]=+\infty
$$

De fato, pois caso contrário $\left(x_{k}\right)$ seria uma sequência Palais-Smale para $\mathcal{L}_{T}$ em $\Omega_{p, q}([0, T], M)$ ao nível

$$
c=\limsup _{k \rightarrow \infty} \mathcal{L}_{T}\left[x_{k}\right]<+\infty,
$$

e portanto, deveria conter uma subsequência convergente a um ponto crítico de $\mathcal{L}_{T}$, solução de (3.1). Como $p$ e $q$ são não-conjugados, $\mathcal{L}_{T}$ não tem pontos críticos degenerados em $\Omega_{p, q}([0, T], M)$, e logo, $\mathcal{L}_{T}$ é uma função Morse. Então todo ponto crítico $x$ de $\mathcal{L}_{T}$ é isolado, o que contradiz a existência de uma solução de (3.1) que seja limite de uma subsequência.

Como, pela definição 3.3,

$$
\mathcal{L}_{T}\left[x_{k}\right]=\int_{0}^{T}\left[\frac{1}{2} g\left(\dot{x}_{k}, \dot{x}_{k}\right)-V\left(x_{k}\right)\right] d t
$$

para cada $k \in \mathbb{N}$ e pelo Lema 3.1, $x_{k}$ satisfaz a Lei de conservação:

$$
\frac{1}{2} g\left(\dot{x}_{k}, \dot{x}_{k}\right)+V\left(x_{k}\right) \equiv E_{k}=\text { cte }
$$

tem-se que:

$$
\begin{aligned}
\mathcal{L}_{T}\left[x_{k}\right] & =\int_{0}^{T}\left(E_{k}-V\left(x_{k}\right)\right)-V\left(x_{k}\right) d t=E_{k} T-2 \int_{0}^{T} V\left(x_{k}\right) d t \\
\Longrightarrow E_{k} & =\frac{1}{T} \mathcal{L}_{T}\left[x_{k}\right]+2 \int_{0}^{T} V\left(x_{k}\right) d t .
\end{aligned}
$$

Então, como $V$ é limitado em $K$ e por (4.2)

$$
\lim _{k \rightarrow \infty} E_{k}=+\infty
$$

Como $g\left(\dot{x}_{k}, \dot{x}_{k}\right)=2\left(E_{k}-V\left(x_{k}\right)\right)$ e $V$ é limitado em $K, g\left(\dot{x}_{k}, \dot{x}_{k}\right)$ é uniformemente divergente em $[0, T]$ isto é,

$$
\lim _{k \rightarrow \infty}\left[\min _{t \in[0, T]} g\left(\dot{x}_{k}, \dot{x}_{k}\right)\right]=+\infty .
$$

Fazendo $b_{k}=\left\|\dot{x}_{k}\left(\frac{T}{2}\right)\right\|$, segue que:

$$
\lim _{k \rightarrow \infty} b_{k}=+\infty \text {. }
$$

Denote por $y_{k}:\left[-b_{k}, b_{k}\right] \rightarrow M$ a reparametrização de $x_{k}$ dada por:

$y_{k}(s)=x_{k}\left(\frac{T}{2}+s \frac{T}{2 b_{k}}\right)=x_{k}(t)$. Daí, $\dot{y}_{k}(s)=\dot{x}_{k}\left(\frac{T}{2}+s \frac{T}{2 b_{k}}\right) \cdot \frac{T}{2 b_{k}}$ e logo,

$$
\begin{aligned}
& \left\{\begin{array}{c}
\dot{y}_{k}(0)=\dot{x}_{k}\left(\frac{T}{2}\right) \cdot \frac{T}{2 b_{k}} \\
\left\|\dot{y}_{k}(0)\right\|=b_{k} \frac{T}{2 b_{k}}=\frac{T}{2}
\end{array} \quad \mathrm{e}\right. \\
& \frac{D}{d s} \dot{y}_{k}(s)=\frac{D}{d t} \dot{x}_{k}(t)\left(\frac{T}{2 b_{k}}\right)^{2} .
\end{aligned}
$$


As hipóteses do Lema 4.1 são satisfeitas para a sequência $y_{k}$ pois, por (4.4)

$$
\dot{y}_{k}(0)=\frac{T}{2} \frac{\dot{x}_{k}\left(\frac{T}{2}\right)}{\left\|\dot{x}_{k}\left(\frac{T}{2}\right)\right\|}
$$

e pode-se assumir que, além de subsequências, $\dot{y}_{k}(0)$ converge para um vetor nãonulo $v_{0} \in T_{y(0)} M$, e como

$$
\left\|\frac{D}{d t} \dot{x}_{k}(t)\right\|=\left\|\nabla V\left(x_{k}(t)\right)\right\|
$$

é limitada, segue, por (4.3) e (4.5), que $\left\|\frac{D}{d s} \dot{y}_{k}(s)\right\|$ converge uniformemente a 0 em cada subconjunto compacto de $\mathbb{R}$. Portanto, pelo Lema 4.1, conclui-se que $y_{k}$ converge, em cada intervalo limitado de $\mathbb{R}$, para uma geodésica não-constante, com parametrização afim, $y: \mathbb{R} \rightarrow M$.

Como $y_{k}$ tem imagem em $K$ para todo $k \in \mathbb{N}$, também $y$ tem imagem em $K$, isto é, $y(s)$ é limitada em $M$. Como, pela Proposição 2.35, $F$ é contínua em $M$, em particular $F(y(s))$ é limitada.

Mas a função $F \circ y: \mathbb{R} \rightarrow \mathbb{R}$ é estritamente convexa, pois por hipótese assumiuse que $F$ o é, e portanto, pelo Lema 2.20 , não pode ser limitada superiormente ou seja,

$$
\lim _{s \rightarrow \infty} F(y(s))=+\infty \text { ou } \lim _{s \rightarrow-\infty} F(y(s))=+\infty .
$$

Obtem-se uma contradição cujo absurdo está em supor-se inicialmente a existência de $\left(x_{k}\right)_{k=1}, \cdots, \infty$ contida em $K$, compacto de $M$. Portanto o número de soluções $x_{k}$ em $K$ é finito.

Analisa-se agora sob que condições tem-se finitude no número de todas as soluções do problema (I). O resultado central nesta direção está baseado num controle adequado do crescimento ao infinito da função convexa $F$ ao longo das linhas de fluxo de $\nabla V$. Na verdade, só é possível passar do resultado "local" para o "global", quando os sistemas dinâmicos tem a seguinte propriedade: todas as soluções com extremos dentro de um conjunto compacto não saem de um subconjunto compacto de $M$, possivelmente maior. Tais equações diferenciais são definidas como regulares em algumas referências, como por exemplo em Peixoto[16]:

DEFINIÇÃo 4.6. A equação diferencial

$$
\ddot{x}=f(t, x, \dot{x})
$$

é dita regular se para todo subconjunto compacto $K_{1} \subset \mathbb{R}^{n}$ existe um subconjunto compacto $K_{2} \subset \mathbb{R}^{n}$ tal que todas as soluções de (4.6), $x:[a, b] \rightarrow \mathbb{R}^{n}$ satisfazendo $x(a), x(b) \in K_{1}$ permanecem dentro de $K_{2}$. 
Assim, nesta linguagem, a existência de uma função estritamente convexa em $M$ que é não-crescente nas linhas de fluxo de $\nabla V$ é uma condição suficiente para garantir a regularidade do sistema dinâmico, como será demonstrado a seguir. Primeiro mostra-se um resultado que, para ser mais claro, usa hipóteses ainda não as mais satisfatórias, mas que serão enfraquecidas posteriormente.

PROPOSIÇÃo 4.7. Sejam $(M, g)$ uma variedade Riemanniana completa, $V: M \rightarrow \mathbb{R}$ uma aplicação $C^{2}$ e assuma que $M$ admite uma função fortemente convexa tal que: $F$ tem um ponto de mínimo em $M$ e $F$ é não-crescente em toda linha de fluxo do $\nabla V$. Se p e q são não-conjugados sobre $[0, T]$ relativamente a (3.1). então existe apenas um número finito de soluções do problema (I).

Prova. Usa-se a Proposição 4.5, mostrando que, sob estas hipóteses, todas as soluções de (I) devem permanecer dentro de algum subconjunto compacto de $M$. Observe que:

(1) Seja $\gamma: \mathbb{R} \rightarrow M$ uma curva integral maximal de $\nabla V$ em $M$ (Definição 1.68). Se $\left.F\right|_{\gamma(t)}$ é não-crescente então $\frac{d}{d t}(F \circ \gamma)(t)=g(\nabla V(\gamma(t)), \dot{\gamma}(t)) \leq 0$ o que implica em $g(\nabla F, \nabla V) \leq 0$ em $M$. Afirma-se, então, que: $F \circ x:[0, T] \rightarrow \mathbb{R}$ é estritamente convexa para toda solução não-constante $x:[0, T] \rightarrow M$ de (3.1). De fato, para cada solução $x$, calcula-se:

$$
\begin{aligned}
\frac{d^{2}}{d t^{2}} F(x(t)) & =g\left(\nabla F(x), \frac{D}{d t} \dot{x}\right)+\operatorname{Hess} F(x(t))[\dot{x}(t), \dot{x}(t)] \\
\Longrightarrow \quad \frac{d^{2}}{d t^{2}} F(x(t)) & =-g(\nabla F(x), \nabla V(x))+\operatorname{Hess} F(x(t))[\dot{x}(t), \dot{x}(t)] .
\end{aligned}
$$

Por hipótese $F: M \rightarrow \mathbb{R}$ é fortemente convexa, ou seja, pela definição (2.34), existe $\lambda: M \rightarrow \mathbb{R}^{+}$contínua tal que Hess $F(x)(v, v) \geq \lambda(x) g(v, v)$ para toda $x$ em $M$ e todo $v \in T_{x(t)} M$. Como $-g(\nabla F(x), \nabla V(x)) \geq 0, \frac{d^{2}}{d t^{2}} F(x(t))$ é estritamente positivo exceto possivelmente nos zeros de $\lambda$ e naqueles instantes em que $\dot{x}(t)=0$. Como toda solução não-constante $x$ de (3.1) apenas admite zeros isolados da derivada $\dot{x}$, este é um conjunto discreto. Portanto $F \circ x$ é estritamente convexa;

(2) Como $F$ é fortemente convexa em $M$, este ponto de mínimo é necessariamente não-degenerado e logo, para todo $c \in \mathbb{R}, F^{c}=\{x \in M: F(x) \leq c\}$ é compacto em $M$ (Corolário 2.40);

(3) Funções estritamente convexas em intervalos fechados atingem seu máximo em um dos extremos (Lema 2.22).

Então toda solução de (3.1) tem imagem em um subconjunto compacto $F^{d}$ onde $d=\max \{F(p), F(q)\}$ ou seja, $x([0, T]) \subset F^{d}$ para toda solução $x:[0, T] \rightarrow M$ de (3.1), satisfazendo (3.2). Portanto, pela Proposição 4.5 existe apenas um número finito de soluções de (3.1), satisfazendo (3.2). 
OBSERVAÇÃO 4.8. Da prova da Proposição 4.7 fica claro que a mesma conclusão ocorre com a condição mais fraca de que $F$ seja não-decrescente ao longo das linhas de fluxo de $\nabla V$ apenas fora de algum subconjunto compacto de $M$.

Observe que o resultado finito da Proposição anterior ocorre sob a hipótese mais geral da existência de uma função própria $F: M \rightarrow \mathbb{R}$, não necessariamente diferenciável, tal que $F$ ○ $x$ é estritamente convexa para toda solução não-constante de (3.1). Então, nesta nova prova, se $d=\max \{F(p), F(q)\}$, então $x([0, T]) \subset F^{d}$ compacto em $M$ para toda solução $x:[0, T] \rightarrow M$ não-constante de (3.1) pois, $F$ é própria e logo, ilimitada ao infinito (Corolário 2.40).

Como consequência da Proposição 3.15 e da Proposição anterior (4.7), finalmente mostra-se que $\sigma_{\infty}$ tem medida nula em $M$ (Definição 3.2), sob as mesmas hipóteses da Proposição 4.7:

COROLÁRIO 4.9. Se $(M, g)$ é uma variedade Riemanniana completa, $V: M \rightarrow \mathbb{R}$ é uma aplicação $C^{2}, M$ admite uma função fortemente convexa $F$ : $M \rightarrow \mathbb{R}$ tal que: $F$ tem um ponto de mínimo em $M$ e $F$ é não-crescente em toda linha de fluxo do $\nabla V$ e p e q são não-conjugados sobre $[0, T]$ relativamente a (3.1). Então o conjunto $\sigma_{\infty}$ tem medida nula em $M$.

Prova. $\sigma_{\infty}$, pela Proposição 4.7, é um conjunto de pontos de $M$ que são conjugados a $p$ pois, caso contrário o conjunto das trajetórias $x_{k}$ seria finito e logo não se trataria de $\sigma_{\infty}$. Portanto, pela Proposição 3.15, este conjunto, o dos pontos $q \in M$ que são conjugados a $p$ sobre $[0, T]$ relativamente a (3.1), tem medida nula em $M$.

\section{Finitude de soluções do problema diferencial em que o potencial é nulo}

Se o potencial $V$ é nulo então o sistema dinâmico (3.1) reduz-se à equação de geodésicas em $(M, g)$ :

(4.7) $\frac{D \dot{x}}{d t} \equiv 0$.

Fixados os pontos $p$ e $q$ em $M$, interessa estudar as soluções $x:[0,1] \rightarrow M$ de (4.7) que satisfazem as condições de fronteira:

$$
x(0)=p \text { e } x(1)=q .
$$

Neste caso, a Proposição 4.7 dá-nos um resultado finito para geodésicas ligando $p$ e $q$, dois pontos não-conjugados em $M$, que admite uma função fortemente convexa com um ponto de mínimo.

A prova direta deste resultado de finitude para geodésicas também é interessante e será feita a seguir. Para tanto são necessários dois resultados gerais, de interesse independente, apresentados nos seguintes lemas. 
LEMA 4.10. Se p e q são não-conjugados em $M$ então o conjunto das geodésicas ligando $p$ a qé discreto em $M$.

Prova. Como $p$ e $q$ são não-conjugados, pelo Lema 1.50, $q$ é valor regular de $\exp _{p}$ e logo $\left(\operatorname{dex} p_{p}\right)_{v}$ é um isomorfismo para todo $v \in \exp p_{p}^{-1}(q)$. Pelo Teorema da Função Inversa, existem abertos $\mathcal{U}_{v} \subset T_{p} M$ e $U \subset M \operatorname{com} q \in U$ tal que $\exp _{p}: \mathcal{U}_{v} \rightarrow U$ é bijetora. Segue que $\exp _{p}^{-1}(q) \cap \mathcal{U}_{v}=\{v\}$ ou seja, $v$ é um ponto isolado de $\exp _{p}^{-1}(q)$ e portanto $\exp _{p}^{-1}(q)$ é um conjunto discreto. Como existe uma correspondência biunívoca entre $\exp _{p}^{-1}(q)$ e $\Gamma=\left\{x \in \Omega_{p, q}^{1}, x:[0,1] \rightarrow M \mid x\right.$ é geodésica\}, $\Gamma$ é um conjunto discreto.

Este resultado também pode ser mostrado considerando-se que, como os pontos críticos do funcional Energia $E \operatorname{em} \Omega_{p, q}^{1}$ são justamente as geodésicas $\gamma \in \Gamma \subset \Omega_{p, q}^{1}$, e para $p$ e $q$ não-conjugados em $M$, todo ponto crítico de $E$ é não-degenerado, $E$ é uma função de Morse (Definição 4.2). Portanto, como todo ponto crítico de uma fụnção de Morse é isolado, o conjunto $\Gamma$ é discreto.

LEMA 4.11. O limite uniforme de uma sequência de geodésicas normalizadas $y_{n}:[a, b] \rightarrow$ M também é uma geodésica normalizada.

PROVA. Como a sequência $\left(y_{n}\right)$ converge uniformemente a uma curva contínua $y:[a, b] \rightarrow M$, em particular pontualmente

$$
\lim _{n \rightarrow \infty} y_{n}(a)=\lim _{n \rightarrow \infty} p_{n}=p_{0}=y(a) .
$$

Como $\left\|\dot{y}_{n}(a)\right\|=1$ para todo $n \in \mathbb{N}$, a sequência $\left(\dot{y}_{n}(a)\right)$ permanece em um subconjunto compacto de $T M$ e pode-se assumir que, além de subsequências,

$$
\lim _{n \rightarrow \infty} \dot{y}_{n}(a)=\lim _{n \rightarrow \infty} v_{n}=v_{0}=\dot{y}(a) \in T_{p_{0}} M
$$

$\operatorname{com} v_{0}$ unitário pois $\left|\left\|\dot{y}_{n}(a)\right\|-\left\|v_{0}\right\|\right| \leq\left\|\dot{y}_{n}(a)-v_{0}\right\| \rightarrow 0$ quando $n \rightarrow \infty$.

Por outro lado, cada $y_{n}$ é solução do problema de Cauchy:

$$
\nabla_{y_{n}} \dot{y_{n}} \equiv 0, y_{n}(a)=p_{n} \text { e } \dot{y_{n}}(a)=v_{n}
$$

e usando a dependência contínua entre os dados iniciais de uma equação diferencial ordinária e suas soluções, pode-se tomar o limite quando $n \rightarrow \infty$, obtendo que o limite uniforme de $y_{n}$, dado por $y$, é solução de $\nabla_{\dot{y}} \dot{y} \equiv 0$ satisfazendo as condições iniciais $y(a)=p_{0}$ e $\dot{y}(a)=v_{0}$. Portanto $y$ é uma geodésica normalizada.

PROPOSIÇão 4.12. Seja $(M, g)$ uma variedade Riemanniana completa. Seja $F: M \rightarrow \mathbb{R}$ uma função de classe $C^{2}$. Assuma que Hess $F(p)$ é definido positivo para todo $p \in M$ e que $F$ admite um ponto de mínimo não-degenerado. Se $p$ e q são pontos não-conjugados em $M$ então existe apenas um número finito de geodésicas em $M$ ligando $p$ a $q$. 
Prova. Seja $\Gamma=\left\{x \in \Omega_{p, q}^{1}, x:[0,1] \rightarrow M \mid x\right.$ é geodésica $\}$.

Em primeiro lugar, observe que existe um subconjunto compacto $K$ de $M$ que contém a imagem de cada geodésica ligando $p$ a $q$.

Como Hess $F$ é definido positivo em $M$, pela Observação 2.33, $F$ é estritamente convexa em $M$ e logo, por definição (2.28), $F \circ x:[0,1] \rightarrow \mathbb{R}$ é estritamente convexa em $[0,1]$ para toda $x \in \Gamma$. Seja $d=\max \{F(p), F(q)\}$ então $F(x(t)) \leq d$, para todo $t \in[0,1]$ ou seja, $x([0,1]) \subset F^{d}$ para toda $x \in \Gamma$, pois funções estritamente convexas assumem valor máximo nos extremos do intervalo de definição (Lema 2.22). Como Hess $F$ é definido positivo, $F$ admite um ponto de mínimo necessariamente nãodegenerado e segue, pelo Corolário 2.40, que $F^{d}$ é compacto em $M$. Portanto basta tomar $K=F^{d}$.

Afirma-se que existe uma constante $c_{o}$ tal que $g(\dot{x}, \dot{x}) \leq c_{o}$ para toda $x \in \Gamma$.

De fato, pois caso contrário, dado $c>0$, existe $x \in \Gamma$ tal que $g(\dot{x}, \dot{x})>c$. Quer dizer, existe uma sequência $x_{n}:[0,1] \rightarrow M$ de geodésicas em $M, x_{n} \in \Gamma$, tal que $g\left(\dot{x}_{n}, \dot{x}_{n}\right)=c_{n} \rightarrow \infty$ quando $n \rightarrow \infty$.

Seja $y_{n}=x_{n} \circ \phi$, uma reparametrização de $x_{n}$ por comprimento de arco, onde $\phi:\left[0, \sqrt{c_{n}}\right] \rightarrow[0,1]$ é dado por $\phi(s)=t=\frac{s}{\sqrt{c_{n}}}$.

Defina a sequência $\tilde{y_{n}}:[0, \infty[$ de geodésicas normalizadas por

$\tilde{y_{n}}=\left\{\begin{array}{lll}y_{n} & \text { se } & s \in\left[0, \sqrt{c_{n}}\right] \\ q & \text { se } & s>\sqrt{c_{n}}\end{array}\right.$

Como os traços de $x_{n}([0,1]), y_{n}\left(\left[0, \sqrt{c_{n}}\right]\right)$ e $\tilde{y_{n}}([0, \infty[)$ coincidem, a sequência $\tilde{y}_{n}$ é uniformente limitada, pois tem imagem em $K$, compacto de $M$.

Como $\left\|\tilde{y_{n}}{ }^{\prime}(s)\right\|=1$ para todo $s>0$ e todo $n \in \mathbb{N}$, $\tilde{y_{n}}$ é um conjunto equicontínuo isto é, dado $\epsilon>0$ existe $\delta=\epsilon>0$ tal que se $\left|s_{1}-s_{2}\right|<\delta$ então $\operatorname{dist}\left(\tilde{y_{n}}\left(s_{1}\right), \tilde{y_{n}}\left(s_{2}\right)\right)=\left|s_{2}-s_{1}\right|<\delta=\epsilon$ para todo $s_{1}, s_{2}>0$ e todo $n \in \mathbb{N}$.

Pelo Teorema de Ascoli-Arzelá, toda sequência de funções equicontínua e limitada ponto a ponto, possui uma subsequência uniformemente convergente em cada parte compacta de $[0, \infty[$. E também, como o limite uniforme de uma sequência de funções contínuas é contínuo, além de subsequências, $\tilde{y_{n}}$ é uniformemente convergente em subconjuntos compactos de $[0, \infty[$ a uma curva contínua $y:[0, \infty[\rightarrow M$.

Além disso, pelo Lema 4.11, $y:[0, \infty[\rightarrow M$ é uma geodésica normalizada; e como as $y_{n}$ 's tem imagem em $K$, também $y$ tem imagem em $K$.

Por outro lado, como $F$ tem um ponto crítico não-degenerado, pela Proposição 2.39, $F$ é ilimitada ao infinito ou seja,

$$
\lim _{s \rightarrow \infty} F(y(s))=+\infty
$$


o que contradiz o fato de $y$ estar contida no compacto $K$ de $M$ pois, como $F$ é contínua, teria imagem em um compacto de $\mathbb{R}$. Portanto a energia de toda geodésica ligando $p$ a $q$ é limitada.

Conclui-se que $\Gamma$ é um conjunto limitado em $M$, e então, $\Gamma$ é compacto em $M$. De fato, como qualquer sequência de geodésicas em $\Omega_{p, q}^{1}$ deve ter uma subsequência convergente a um limite uniforme que também é uma geodésica em $\Omega_{p, q}^{1}$, por definição, $\Gamma$ é fechado pois contem todos seus pontos de aderência. Pelo Teorema de HopfRinow (1.56), $\Gamma$ limitado e fechado, é compacto em $M$ completa.

Como $p$ e $q$ são não-conjugados em $M$, pelo Lema 4.10, $\Gamma$ é discreto em $M$. Portanto, como todo subconjunto compacto e discreto é finito, conclui-se que existe apenas um número finito de geodésicas ligando $p$ a $q$ em $M$.

\section{Outros critérios de regularidade do sistema dinâmico (3.1) e Exemplos}

Aqui são discutidos outros critérios de regularidade do sistema dinâmico (3.1), dados nos lemas 4.14 e 4.16 , assim como exemplos em $\mathbb{R}^{n}$ onde esta teoria se aplica.

Se $(M, g)$ é o espaço euclidiano $\mathbb{R}^{n}$ então existem vários critérios para estabelecer quando o sistema dinâmico (3.1) definido em $\mathbb{R}^{n}$

$$
\ddot{x}=-\nabla V(x)
$$

é tal que todas as suas soluções $x:[0, T] \rightarrow \mathbb{R}^{n}$ que satisfazem as condições de fronteira

$$
x(0)=p \text { e } x(T)=q
$$

permanecem dentro de um compacto de $\mathbb{R}^{n}$ ou seja, critérios que garantem a regularidade do sistema segundo a Definição 4.6.

Estuda-se dois critérios de regularidade para (4.9): o primeiro dado no lema a seguir e o segundo no Lema 4.15.

LEMA 4.13. Considere o sistema dinâmico (3.1) em $\mathbb{R}^{n}$, com $V: \mathbb{R}^{n} \rightarrow \mathbb{R}$, uma função de classe $C^{2}$. Assuma que $\|\nabla V\|$ é limitada. Ėntão toda solução de (4.9) satisfazendo (4.10) tem imagem em um subconjunto compacto de $\mathbb{R}^{n}$.

Prova. Como $\|\nabla V\|$ é limitada, existe $L>0$ tal que: $\|\nabla V\| \leq L$ para toda solução $x$ de (4.9) e (4.10).

Denote por $x_{i}:[0, T] \rightarrow \mathbb{R}$ a $i$-ésima coordenada de $x \mathrm{e}$ seja $t_{i} \in[0, T]$ um ponto de máximo de $\left|x_{i}\right|$. Segue que $\left|x_{i}(t)\right| \leq\|x(t)\| \leq \sqrt{n}\left|x_{i}(t)\right| \leq \sqrt{n}\left|x_{i}\left(t_{i}\right)\right|$. 
Fazendo $q=\left(q_{1}, \cdots, q_{n}\right)$, tem-se:

$$
\begin{aligned}
\left|q_{i}-x_{i}\left(t_{i}\right)\right| & =\left|\int_{t_{i}}^{T} \dot{x}_{i}(s) d s\right| \\
& =\left|\int_{t_{i}}^{T}\left(\int_{t_{i}}^{s} \ddot{x}_{i}(r) d r\right) d s\right| \leq \int_{t_{i}}^{T} \int_{t_{i}}^{s}\left|\ddot{x}_{i}(r)\right| d r d s \\
& \leq \int_{t_{i}}^{T} \int_{t_{i}}^{s}\|\ddot{x}(r)\| d r d s=\int_{t_{i}}^{T} \int_{t_{i}}^{s}\|\nabla V(x(r))\| d r d s \\
& \leq \int_{t_{i}}^{T} \int_{t_{i}}^{s} L d r d s=\int_{t_{i}}^{T} L\left(s-t_{i}\right) d s=\left.\left(L \frac{s^{2}}{2}-L t_{i} s\right)\right|_{t_{i}} ^{T} \\
& \leq L \frac{T^{2}}{2} \leq L T^{2}
\end{aligned}
$$

Então ||$q-x(t)\|=\| x(T)-x(t) \| \leq \sqrt{n}\left|q_{i}-x_{i}\left(t_{i}\right)\right| \leq \sqrt{n} L T^{2}$. Portanto todas as soluções de (4.9) que satisfazem (4.10) tem imagem na bola fechada centrada em $q$ com raio $L T^{2} \sqrt{n}$.

Conclui-se que, dado o sistema dinâmico (4.9) em $\mathbb{R}^{n}: \ddot{x}=-\nabla V(x)$, e usandose o critério acima ou seja, se $\|\nabla V\|$ é limitada, assegura-se que toda solução permanece em algum compacto de $\mathbb{R}^{n}$ (resultado análogo ao da Proposição 4.7). E, substituindo-se o critério de convexidade por hipóteses adequadas sobre o crescimento de $\|\nabla V\|$, a Proposição 4.5 garante que existe apenas um número finito de soluções de (4.9) ligando dois pontos nạ̃o-conjugados.

Um exemplo de tal sistema é dado pela equação do pêndulo: $\ddot{x}=-\operatorname{sen} x$ em $\mathbb{R}$.

No caso geral, dado pela equação diferencial (4.6) $\ddot{x}=f(t, x, \dot{x})$, se $f$ é limitada, então o mesmo argumento do Lema 4.13 mostra que (4.6) é regular.

O seguinte critério estabelece também a regularidade do sistema (3.1) em $M$ :

LEMA 4.14. Dado o problema diferencial (I) em uma variedade Riemanniana completa $(M, g)$, suponha que:

(1) $\lim _{x \rightarrow \infty} V(x)=-\infty$;

(2) V não tem pontos críticos fora de qualquer subconjunto compacto de $M$;

(3) $O$ hessiano Hess $V$ é semi-definido negativo na distribuição ortogonal $\nabla V^{\perp}$ fora de qualquer subconjunto compacto de $M$.

Então todas as soluções $x:[0, T] \rightarrow M$ de (3.1) que satisfazem (3.2) tem imagem dentro de um subconjunto compacto de $M$.

PROVA. Como, por (1), $\lim _{x \rightarrow \infty} V(x)=-\infty$, os conjuntos fechados $V_{c}=\{x \in M: V(x) \geq c\}=V^{-1}([c,+\infty[)$ são compactos para todo $c \in \mathbb{R}$. 
A prova será feita mostrando que todas soluções de (3.1) e (3.2) permanecem em algum $V_{c}$.

Suponha, por absurdo, que existe $x:[0, T] \rightarrow M$ uma solução de (3.1) tal que para algum $\tau \in[0, T], x_{0}=x(\tau) \notin V_{c}$ ou seja,

$$
V\left(x_{0}\right)<c \leq V(x) \Longrightarrow V(x(\tau)) \leq V(x(t))
$$

para todo $t \in[0, T], t \neq \tau$.

Logo $\tau$ é um ponto de mínimo para $h(t)=V(x(t)) \Longrightarrow h^{\prime}(\tau)=0$.

Podemos tomar, é claro, $\tau \in] 0, T[$.

Observe que $x_{0}$ não é ponto crítico de $V$, pois, por (2), $V$ não possui pontos crítiços fora de qualquer compacto de $M$. Então $\nabla V\left(x_{0}\right) \neq 0$.

Calculando:

$$
\begin{aligned}
& h^{\prime}(t)=g(\nabla V(x(t)), \dot{x}(t)) \text { e logo, } \\
& h^{\prime}(\tau)=g(\nabla V(x(\tau)), \dot{x}(\tau))=0 \Longrightarrow \dot{x}(\tau) \in \nabla V(x(\tau))^{\perp}
\end{aligned}
$$

e como neste caso Hess $V(x(\tau))$ é semi-definido negativo em $\dot{x}(\tau)$ por (3), tem-se:

$$
\begin{aligned}
h^{\prime \prime}(\tau) & =\operatorname{Hess} V(x(\tau))[\dot{x}(\tau), \dot{x}(\tau)]+g\left(\nabla V(x(\tau)), \frac{D}{d t} \dot{x}(\tau)\right) \\
& =\operatorname{Hess} V(x(\tau))[\dot{x}(\tau), \dot{x}(\tau)]-\|\nabla V(x(\tau))\|^{2}<0 .
\end{aligned}
$$

Então $\tau$ é ponto de máximo de $h$ e chegamos a uma contradição com a hipótese inicial de que $\tau$ fosse ponto de mínimo de $h$. Isto conclui a prova.

Para sistemas dinâmicos em espaços euclidianos, temos também o seguinte critério de regularidade, baseado no bem conhecido caso uni-dimensional, dependente do tempo, e aqui aplicado ao sistema (4.9): $\ddot{x}(t)=-\nabla V(x(t))$.

LEMA 4.15. Considere o sistema dinâmico (3.1) em $\mathbb{R}^{n}$, com $V: \mathbb{R}^{n} \rightarrow \mathbb{R}$, uma função de classe $C^{2}$ satisfazendo a condição:

$$
x_{i} \frac{\partial V}{\partial x_{i}}<0
$$

para todo $i=1, \cdots$, n e para todo $x_{i}$ com $\left|x_{i}\right|$ suficientemente grande.

Então toda solução de (4.9) satisfazendo (4.10) tem imagem em um subconjunto compacto de $\mathbb{R}^{n}$.

ProvA. Seja $x=\left(x_{1}, \cdots, x_{n}\right):[0, T] \rightarrow \mathbb{R}^{n}$ uma solução de (4.9) e (4.10). Para todo $i=1, \cdots, n$, seja $t_{i} \in[0, T]$ um ponto de máximo para $x_{i}(t)$. Podemos assumir que $\left.t_{i} \in\right] 0, T\left[\right.$ e $x_{i}\left(t_{i}\right) \neq 0$.

Se $x_{i}\left(t_{i}\right)>0$, o valor de $x_{i}\left(t_{i}\right)$ não pode ser muito grande, pois caso contrário, por (4.11): $\left\langle\nabla V\left(x\left(t_{i}\right)\right), x\left(t_{i}\right)\right\rangle=\sum_{i=1}^{n} x_{i}\left(t_{i}\right) \frac{\partial V}{\partial x_{i}} x_{i}\left(t_{i}\right)<0$ e se $x_{i}\left(t_{i}\right)>0$ para todo $i=1, \cdots, n$, então $\frac{\partial V}{\partial x_{i}}\left(x_{i}\left(t_{i}\right)\right)<0$. Conclui-se que $\ddot{x}_{i}\left(t_{i}\right)=-\nabla V\left(x_{i}\left(t_{i}\right)\right)>0$ e portanto $t_{i}$ seria ponto de mínimo para $x_{i}$. 
Analogamente, se $x_{i}\left(t_{i}\right)<0$, o valor de $-x_{i}\left(t_{i}\right)$ não pode ser muito grande, pois caso contrário, por (4.11) $\frac{\partial V}{\partial x_{i}}\left(x_{i}\left(t_{i}\right)\right)>0 \Longrightarrow \ddot{x}_{i}\left(t_{i}\right)=-\nabla V\left(x_{i}\left(t_{i}\right)\right)<0$ e $x\left(t_{i}\right)$ não poderia ser um mínimo para $x_{i}(t)$.

Logo existe $K \in \mathbb{R}$ tal que $\left|x_{i}\left(t_{i}\right)\right| \leq K$.

Segue que: $\|x(t)\| \leq \sqrt{n}\left|x_{i}(t)\right| \leq \sqrt{n}\left|x_{i}\left(t_{i}\right)\right| \leq \sqrt{n} K$ e a prova está concluída.

Exemplos de potenciais $V$ satisfazendo as condições do Lema acima são todas as funções polinomiais em $\mathbb{R}^{n}$, contendo apenas potências pares das variáveis e cujos coeficientes são negativos.

Voltando ao caso geral dos sistemas definidos em variedades Riemannianas, se $M$ admite uma função fortemente convexa $F$ então a Proposição 4.7 pode ser aplicada ao caso em que $V=-F$. (Uma função $F: M \rightarrow \mathbb{R}$ é concava se $-F$ é convexa). De fato, neste caso, a condição de convexidade pode ser enfraquecida e obtem-se o seguinte resultado finito para potenciais concavos.

Proposição 4.16. Sejam $(M, g)$ uma variedade Riemanniana completa e $V: M \rightarrow \mathbb{R}$ uma função de classe $C^{2}$ fortemente concava e com um máximo isolado. Então o sistema dinâmico (3.1) admite apenas um número finito de soluções $x:[0, T] \rightarrow M$ satisfazendo (3.2), desde que $p$ e q sejam não-conjugados sobre $[0, T]$ relativamente a $(3.1)$.

Prova. A prova da Proposição 4.7 pode ser aqui repetida colocando-se $F=-V$, exceto pelo seguinte detalhe:

Dada uma solução $x:[0, T] \rightarrow M$ a convexidade estrita de $F \circ x$ é agora provada, não pela forte convexidade de $F$ mas observando que Hess $F(x)[\dot{x}, \dot{x}] \geq 0$ enquanto - $g(\nabla F(x), \nabla V(x))=g(\nabla V(x), \nabla V(x))>0$ exceto possivelmente quando $x$ passa pelo único ponto crítico de $V$.

Então $F$ é estritamente convexa com um ponto de mínimo não-degenerado e portanto $x([0, T]) \subset F^{d}$, compacto de $M$. 


\section{Referências Bibliográficas}

[1] R. L. Bishop, B. O'Neill, "Manifolds of negative curvature”, Trans.Am.Soc. 145 (1969), 1-49.

[2] W. M. Boothby, "An Introduction to Differentiable Manifolds and Riemannian Geometry", Academic Press, New York (1986).

[3] M. P. do Carmo, "Geometria Riemanniana", IMPA-CNPq, Rio de Janeiro (1988). $2^{a}$ edição.

[4] L. Fichmann, E. M. Sallum, "Sistemas Dinâmicos: Noções Básicas”, IME-USP, São Paulo (2001).

[5] F. Giannoni, A. Masielo, P. Piccione, "Convexity and the Finiteness of the Number of Geodesics. Applications to the Multiple-Image Effect", Class. Quantum Grav. 16(1999), 731-748.

[6] F. Giannoni, A. Masielo, P.Piccione, "Convexity and Finiteness of the number of solutions for two-points boundary value problem in Riemannian Manifolds", Preprint.

[7] F. Giannoni, A. Masielo, P. Piccione, "On the Finiteness of Light Rays between a Source and an Observer for Conformally Stationary Spacetimes", General Relativity and Grav., 33(3), (2001), 491-514.

[8] R. E. Green, K. Shiohama, “Convex functions on complete noncompact Riemannian manifolds: Topological Structure”, Inventions Math.63 (1981), 129-157.

[9] E. L. Lima, "Curso de Análise”, vol.1, IMPA-CNPq, Rio de Janeiro (1982).

[10] E. L. Lima, "Curso de Análise",vol.2, IMPA-CNPq, Rio de Janeiro (2000).

[11] S. X. Mendonça, P. Piccione, "Convexity, curvature and geodesics in Riemannian Geometry". Preprint.

[12] J. Milnor, “Morse Theory”, Princeton U. Press, New Jersey-USA (1973).

[13] B. O'Neill,"Semi-Riemannian Geometry", Academic Press, Florida-USA (1983).

[14] R. Palais, C. Terng, "Critical Point Theory and Submanifold Geometry", Lecture Notes in Mathematics 1353, Springer-Verlag, Berlin (1988).

[15] R. Palais, "Morse Theory on Hilbert Manifolds", Topology 2 (1963), 299-340.

[16] M. M. Peixoto, A. R. da Silva, "Focal decomposition and some results of S. Bernstein on the two-points boundary value problems", J. London Math. Soc. (2) 60 (1999), 517-547.

[17] M. M. Peixoto, "On End-point Boundary value problem", J. Differential Equations 44 (1982), 273-280.

[18] H. Sagan, "Introduction to the Calculus of Variations", Dover, New York (1992).

[19] C. Udriste, "Convex Functions and Optimization Methods on Riemannian Manifolds", Kluwer (1994). 